\title{
Information reliability shaping parasitoid foraging behaviour
}

\section{Jessica A.C. de Bruijn}





\section{Information reliability shaping parasitoid foraging behaviour}

Jessica A.C. de Bruijn 


\section{Thesis committee}

\section{Promotor}

Prof. Dr L.E.M. Vet

Special professor Evolutionary Ecology

Personal chair at the department of Terrestrial Ecology

Netherlands Institute of Ecology (NIOO-KNAW), Wageningen

\section{Co-promotors}

Dr H.M. Smid

Researcher at the Laboratory of Entomology

Wageningen University \& Research

Dr J.G. de Boer

Postdoc researcher at the department of Terrestrial Ecology

Netherlands Institute of Ecology (NIOO-KNAW), Wageningen

\section{Other members}

Prof. Dr M. Naguib, Wageningen University \& Research

Prof. Dr J. Ellers, VU University, Amsterdam

Prof. Dr B. Wertheim, University of Groningen

Prof. Dr J.C. Biesmeijer, Leiden University

This research was conducted under the auspices of the C.T. de Wit Graduate School for Production Ecology \& Resource Conservation. 


\title{
Information reliability shaping parasitoid foraging behaviour
}

\author{
Jessica A.C. de Bruijn
}

\section{Thesis}

submitted in fulfilment of the requirements for the degree of doctor at Wageningen University by the authority of the Rector Magnificus,

Prof. Dr A.P.J. Mol, in the presence of the Thesis Committee appointed by the Academic Board to be defended in public on Tuesday 4 February 2020 at 4 p.m. in the Aula. 
Jessica A. C. de Bruijn

Information reliability shaping parasitoid foraging behaviour, 182 pages.

$\mathrm{PhD}$ thesis, Wageningen University, Wageningen, the Netherlands (2020) With references, with summary in English

ISBN 978-94-6395-211-8

DOI https://doi.org/10.18174/506702 


\section{Table of contents}

\section{Chapter 1}

General introduction

Chapter 2

Automated high-throughput individual tracking system for insect behaviour: Applications on memory retention in parasitoid wasps

\section{Chapter 3}

Costs of persisting unreliable memory: Reduced foraging efficiency for free-flying parasitoid wasps in a wind tunnel

\section{Chapter 4}

Monitoring the effects of information reliability on parasitoid foraging behaviour with a multi-camera system in the field

\section{Chapter 5}

Memory extinction and spontaneous recovery shaping parasitoid foraging behaviour

\section{Chapter 6}

Non-host oviposition by the parasitoid Cotesia glomerata and its effects on foraging behaviour

\section{Chapter 7}

General discussion

References

Summary

Acknowledgements

About the author

Publication list 



\section{Chapter 1}

General introduction 
All animals need resources for their growth, maintenance and reproduction, but these resources may be hard to find in dynamic heterogeneous environments (Vet, 2001; van Alphen et al., 2003; van Alphen and Bernstein, 2008). Foraging in such environments requires complex decisions, where animals use information from their environment in the foraging process (van Alphen and Bernstein, 2008; Thiel and Hoffmeister, 2009). By using this information, in the form of olfactory, visual, auditory and tactile cues that are related to the desired resources, animals can adapt their foraging behaviour to local conditions. This can improve their capacity to locate these resources, i.e. improve their foraging efficiency, which is expected to ultimately increase their fitness (Lewis et al., 1975; Vinson, 1976; Vet, 2001; van Baalen and Hemerik, 2008; Ishii and Shimada, 2009). The link between foraging behaviour and fitness is particularly strong in parasitoid wasps, since their ability to find hosts is directly linked to offspring production, and therefore to fitness (Thiel and Hoffmeister, 2009).

\section{Parasitoid- host interactions}

Parasitoids deposit their eggs in or on other organisms, where successful development of the offspring results in the death of the host (Vinson, 1976). Due to this lethal effect, host insects have evolved strategies to be inconspicuous and thus minimize the risk of being detected by parasitoids (Vet et al., 1995). Hosts minimize the risk of being found by parasitoids, by limiting the detection range of cues that reliably predict host presence, i.e. host-derived cues (Vet et al., 1995). Even when potential hosts are found by the parasitoid, they can defend themselves with structural, behavioural and physiological mechanisms (Brodeur and Vet, 1995; Strand and Pech, 1995; Strand and Obrycki, 1996). Physical structures such as hairs can serve as armour, and behavioural defences include attacks on parasitoids that can damage and occasionally even kill it, or escaping parasitism by wiggling away or dropping from the leaf (Strand and Pech, 1995; Brodeur et al., 1996). Even after parasitoid eggs are deposited, hosts can still defend themselves by encapsulating the eggs, a process where haemocytes form an envelope around the eggs, killing them through asphyxiation and the release of necrotizing substances (Strand and Obrycki, 1996; Kraaijeveld et al., 1998). Although these defences limit the host range of most parasitoid species, parasitoids, in turn, have evolved methods to counteract these defences. For example, some parasitoid species in the Braconidae and Ichneumonidae families can inject polydnavirus and/or venom during oviposition to temporarily or permanently suppress the immune system of the host (Strand and Obrycki, 1996; Kraaijeveld et al., 1998). 


\section{Finding hidden hosts}

The inconspicuous nature of hosts may have driven the development of indirect searching strategies, where parasitoids make use of environmental cues that indirectly predict host presence. The best studied example of such a strategy is found in parasitoids of herbivorous hosts that are known to use herbivore-induced plant volatiles (HIPVs), volatiles that are produced by the plant in response to herbivory (Vet et al., 1995). These cues are, however, generally less reliable indicators of host presence, than cues directly derived from the host. HIPVs tend to be more variable, and do not always provide parasitoids with sufficient information to discriminate between suitable and unsuitable host species, so called non-hosts (Vet et al., 1995; Geervliet et al., 1998b; de Rijk et al., 2013). Nevertheless, many parasitoid species are known to respond to HIPVs, and they are thought to use these cues for longrange orientation to herbivore-infested plants, after which the parasitoid needs to locate the host on the plant and decides whether to deposit eggs.

Once a potential host-infested plant is found, parasitoids typically display hovering behaviour, scanning the plant at a distance of several centimetres, after which they usually land near herbivore-damaged plant tissue. Parasitoids then examine the area around the damaged plant tissue, where they may encounter host-derived cues, such as frass, silk, honeydew or other body secretions (Vinson, 1998; Clavijo McCormick et al., 2012). Contact with these cues generally results in arrestment behaviour, where the parasitoid displays intense directed searching (Vinson, 1976). When these direct cues are not derived from a potential host, but from a non-host herbivore, parasitoids are expected to depart from the plant (Vinson, 1976; Bukovinszky et al., 2012). However, there are also examples of parasitoids being arrested by non-host derived cues, including Cotesia kariyai after contacting the faeces of non-host larvae (Acantholeucania loreyi) (Takabayashi and Takahashi, 1990). Host and non-host derived cues can trigger a reflex response, where the parasitoid starts to pierce or probe various objects leading to attacks on host and non-host species (Vinson, 1976; Bukovinszky et al., 2012). For endoparasitoids, which deposit eggs within the host, actual egg deposition depends on the presence of certain internal cues and the absence of deterrents (Vinson, 1998), where haemolymph compounds are thought to trigger egg deposition (Vinson, 1976). These triggers are not always host specific, because egg deposition has also been observed in non-hosts (Arthur et al., 1969; Takasu and Lewis, 2003; Bukovinszky et al., 2012). 


\section{Improving foraging efficiency through associative learning}

Female parasitoids are genetically adapted to respond to cues that have proven to be reliable indicators of host presence over many parasitoid generations. However, these cues may be insufficient to find hosts in environments that are spatially and temporally dynamic within a single parasitoid generation (Takasu and Lewis, 2003). Because parasitoids and their hosts are generally not monophagous (Vet et al., 1990; Strand and Obrycki, 1996), hosts can be present on different plant species and in many different locations within the habitat. In such cases, parasitoid searching efficiency may be reduced when they respond to multiple sources of information, because their brain capacity for simultaneous processing of information is limited (Hoedjes et al., 2011). Through experience, i.e. encounters, with hosts, non-hosts and their traces, parasitoids gain information on local host availability and distribution (Vet et al., 1995; van Baalen and Hemerik, 2008), i.e. they can learn about the host species that are available and on which plant species they occur. Indeed, parasitoids can associate plant species-specific cues, such as HIPVs, with a host encounter, leading to the formation of an associative memory. The information contained in this memory allows the parasitoid to narrow its search image, leading to a more focussed search for cues previously indicating host presence and allowing for temporal specialization, which can improve foraging efficiency (Turlings et al., 1993; van Baalen and Hemerik, 2008; Ishii and Shimada, 2009; Hoedjes et al., 2011).

\section{Tailor-made memories}

Learning, memory formation and the dynamics of these memories have been extensively studied in honeybees (Menzel, 1999), fruit flies (Margulies et al., 2005) and various parasitoid species (Hoedjes et al., 2011). These aspects are part of an insect's preparedness to learn and are thought to be tailor-made to suit the needs of the insect, i.e. it is adaptive in the context of their ecology (Smid and Vet, 2016). Associative memories are dynamic as they gradually form and decay over time, allowing for temporal specialization (Menzel, 1999). Directly after a learning experience, short-term memory (STM) is formed, which decays in minutes to a few hours and can subsequently be replaced by mid-term memory (MTM) and long-term memory (LTM), which require some time to consolidate, but can persist for hours to days (Hoedjes et al., 2011). Whether memory is formed after certain experiences, and the specific dynamics of these memories, are thought to be adaptations to the conditions of the insect's natural environment. These aspects of learning and memory are therefore expected to be highly species- and even population-specific (Menzel, 1999; Raine and Chittka, 2008; Hoedjes et al., 2011). Memory formation and its dynamics are shaped by the balance between costs and benefits and depend 
on the reliability of the information and its reward value, i.e. the value with respect to the animal's fitness (Hoedjes et al., 2011). Cotesia glomerata, for example, forms MTM after a single oviposition in the host Pieris rapae, of which the female butterfly deposits a single egg on isolated plants of different species. The same parasitoid forms LTM when ovipositing in the host Pieris brassicae, a gregarious butterfly that deposits clusters of over 100 eggs in aggregated stands of host plants of the same species (Kruidhof et al., 2012; Smid and Vet, 2016). When finding P. brassicae, the associated plant cues are expected to be highly reliable in predicting host presence, since multiple plants and hosts are expected to be present in the vicinity, while an encounter with $P$. rapae does not necessarily predict the presence of more hosts. Furthermore, although both species are suitable for offspring development, $P$. brassicae is qualitatively superior to $P$. rapae (Harvey, 2000). The same principles apply for the closely related species Cotesia rubecula, which specializes in finding its $P$. rapae host and forms MTM after a single oviposition experience, but when they find this host several times on the same plant species, MTM is replaced by LTM, as the association has proven to be reliable with subsequent encounters (Smid et al., 2007). Although these examples focus on a single learning event, or several learning events with the same host and plant species, parasitoids gather and store information continuously throughout their lifetime. To date, very little is known about how subsequent learning experiences, with different reward values and different environmental cues, shape parasitoid foraging behaviour.

\section{Verifying information reliability over time}

To optimize their foraging efficiency, parasitoids should process and store information in an adaptive and integrative way and act according to the most reliable information available (Hilker and McNeil, 2008). As described above, associative memory can serve as a first indication that, within the current environment, hosts can be found in the vicinity of the associated environmental cues, such as the HIPVs of a particular plant species. If the HIPVs again lead to suitable hosts, the memory is expected to be strengthened and might become more persistent. If, on the other hand, the same HIPVs are not found again, the memory decays over time (Hoedjes et al., 2011). In a third scenario, memorized host-associated cues, such as HIPVs, are encountered again, but lead the parasitoid to plants without hosts. This may happen when a host has developed into a stage that is unsuitable for parasitism, has left the plant or when non-host instead of host herbivores are present. HIPVs are generally plant species-specific, but they are not always specific enough for parasitoids to discriminate between plants induced by host and non-host herbivores (Geervliet et al., 1996). Encounters with unsuitable host stages and non-host herbivores indicate that previously obtained information currently does not reliably predict the presence 
of suitable hosts, i.e. it is unreliable information. Such unreliable information could have fitness consequences when parasitoids waste time searching for hosts on these plants (Vos et al., 2001). To date, little is known about the effects of unreliable information on parasitoid foraging behaviour. However, this information is essential to understand the evolution of parasitoid foraging strategies in natural environments, comprising multiple herbivore and plant species (Desurmont et al., 2018).

In non-parasitoid systems, such as honeybees, behavioural adjustment due to unreliable information have been studied extensively (Eisenhardt et al., 2013). When the expected reward is not encountered upon responding to the associated cues, termed an extinction experience, this can lead to both temporary and permanent behavioural changes (Eisenhardt, 2012). Temporary behavioural alterations are caused by the formation of an extinction memory that temporarily blocks the retrieval of the original memory, the reward memory. Once this blockage disappears over time, the original memory can be retrieved again, a process termed spontaneous recovery (Eisenhardt, 2012). These processes have been studied extensively with vertebrates and bees, albeit mainly in a neuroscientific context in a laboratory setting with artificial rewards and associated cues that lack natural variation (Kaiser et al., 2003; Lagasse et al., 2009; Eisenhardt et al., 2013). To understand the importance of these processes in nature, they should be studied in an ecological context, with naturally variable rewards and environmental cues, such as host- and plant-derived cues, and ideally in the field, which is ultimately the environment where foraging behaviour evolved.

\section{Research objectives}

As outlined above, environments, and the insect and plant communities within, are often spatially and temporally variable, where parasitoids adapt their foraging behaviour to local conditions. Associative learning is one way to adapt to such changing conditions and has been described in many species of parasitoids. The way they learn, i.e. their preparedness to learn, can vary among species and populations. Even after storing information on local conditions, environments can continue to change and parasitoids might discover that an associative memory has become unreliable. They might encounter unsuitable hosts or non-hosts on plants previously associated with host presence. To date, little is known about the consequences of such encounters for host finding and whether or how parasitoids adapt their foraging behaviour accordingly. To understand in detail how individual parasitoids interact with their environment during foraging, advanced observation methods are needed. 
In this thesis, I studied how unreliable memory, and interactions with Mamestra brassicae non-hosts, shaped the foraging behaviour of the parasitoid Cotesia glomerata. I developed advanced observation set-ups and methods, to track individually foraging parasitoids in the laboratory and the field, and used them to test the following hypotheses:

1) Unreliable associative memory reduces parasitoid foraging efficiency

2) Non-host-related extinction experiences alter a parasitoid's response to associated cues

3) Parasitoids change their foraging behaviour after a non-host oviposition

\section{Study system}

\section{Parasitoid species}

In this thesis, I used the parasitoids Nasonia vitripennis (Hymenoptera: Pteromalidae) and Cotesia glomerata (Hymenoptera: Braconidae) (Fig. 1). Both species are well known for their ability to learn and form long-term memory after a single oviposition experience (Smid et al., 2007; Hoedjes et al., 2012).

Nasonia vitripennis is a gregarious pupal ectoparasitoid that parasitizes several fly species, which commonly occur in bird nests, dung and carcasses (Darling and Werren, 1990). I used this parasitoid to study fundamental aspects of associative ovipositional learning with artificial odours, while I selected C. glomerata to further elucidate various aspects of learning in a more natural context, where herbivoreinduced plant volatiles are associated with an oviposition in a host.

Cotesia glomerata is a gregarious larval endoparasitoid that parasitizes the caterpillars of several members of the lepidopteran family Pieridae, with Pieris brassicae as its preferred host in the Netherlands (Brodeur et al., 1996; Geervliet et al., 2000). Female parasitoids have a potential fecundity of 1000-2200 eggs, which are generally deposited in clutches of approximately 25 eggs in first and second instar hosts (Laing and Levin, 1982; Le Masurier, 1991; Smallegange et al., 2008). Successful development of the offspring leads to the death of the host, but only when the female parasitoid can suppress internal host defences. If the eggs are not killed by the host, larvae hatch and feed on the caterpillar's haemolymph until they are fully grown and ready to egress. Once egressed, they spin cocoons for their final transformation into adults. Directly after adult emergence, female parasitoids mate and start their search for hosts, where long-range orientation to host-infested plants is mainly guided by plant volatiles, while locating hosts on these plants is facilitated 
by host-derived short-range cues, such as leaf damage, silk and frass (Laing and Levin, 1982).
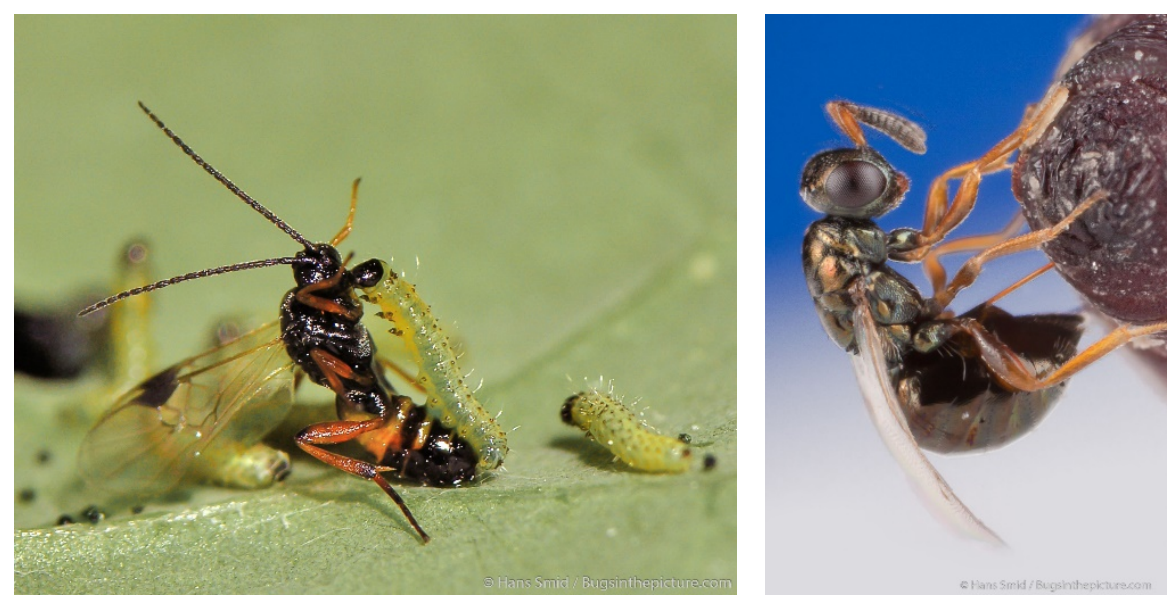

Figure 1. Cotesia glomerata ovipositing in a caterpillar of its Pieris brassicae host on a Brassicae oleracea leaf (left) and Nasonia vitripennis ovipositing in a pupa of its host Calliphora vomitoria (right).

\section{Host and non-host species}

Caterpillars of Pieris brassicae (Lepidoptera: Pieridae) were used as hosts for $C$. glomerata and Mamestra brassicae (Lepidoptera: Noctuidae) caterpillars as nonhosts. Both butterfly species are native to the Netherlands, where they commonly occur on cultivated and wild brassicaceous plants, and their life-history is highly similar (Bell and Muller, 1973; Carter, 1984). They lay large cluster of eggs on their host plants, where early instars feed gregariously and later instars move to other plants in the vicinity (Goulson and Cory, 1995; Vos et al., 1998). For N. vitripennis, the host Calliphora vomitoria (Diptera: Calliphoridae) was used, which is bloodfeeding blowfly species that occurs in bird nests (Darling and Werren, 1990).

\section{Plant species and cultivars}

To attract $C$. glomerata and feed $P$. brassicae and $M$. brassicae caterpillars, I used several members of the Brassicaceae family, including Brassica nigra, Brassica oleracea and Sinapis arvensis. B. nigra (black mustard) and S. arvensis (field mustard) are naturally occurring annual plants in the Netherlands, which commonly occur in grassland vegetation (Bell and Muller, 1973). The B. oleracea plants 
included the agricultural cultivars B. oleracea var. gemmifera cv. Cyrus (Brussels sprouts) and $B$. oleracea var. Rubra cv. Langedijker bewaar (red cabbage).

\section{Thesis outline}

In Chapter 2, I developed the high-throughput individual T-maze, an automated high-throughput system for tracking insect behaviour. With this system, I could simultaneously track the behaviour of 36 individual parasitoids, where each parasitoid was studied in its own T-maze. This method allowed for high-throughput and labour-efficient acquisition of detailed information on individual behaviours of C. glomerata and N. vitripennis with respect to associative learning with artificial odours.

In the following chapters, I switched my focus from the ability to obtain information through associative learning, to the consequences of using this information during foraging. This required testing foraging behaviour in an ecologically more relevant situation, with natural variation in plant volatiles and ideally under field conditions, because this is where parasitoid foraging behaviour has evolved.

In Chapter 3, I tested my first hypothesis. I determined whether unreliable memory had a negative effect on the foraging efficiency of $C$. glomerata. I tested their behaviour in situations where associated HIPVs led parasitoids to a plant species that was infested with non-host caterpillars, while host caterpillars could be found on a plant species that they had no experience with.

In Chapter 4, I extended this study to the field, allowing parasitoids to forage in a more complex natural environment for a substantially longer period of time. I developed a novel multi-camera set-up to observe how parasitoids, with persistent memory containing reliable or unreliable information on host presence, foraged around, and interacted with, host and non-host caterpillars on infested plants.

When parasitoids encounter situations that prove that previously obtained information is unreliable, I expected them to subsequently alter their behaviour to maintain their foraging efficiency. In Chapter 5, I therefore tested whether $C$. glomerata lost its conditioned host plant preference after being given host- and nonhost-related extinction experiences. Due to the high potential fitness value of hostrelated information, I expected host-related extinction experiences to have no effect. In accordance with my second hypothesis, I expected a temporarily suppress of the conditioned host plant preference with non-host-related extinction experiences. 
In previous chapters, I frequently observed that C. glomerata attacked and potentially deposited eggs in the caterpillars of the non-host $M$. brassicae, even under field conditions. This made me wonder whether M. brassicae was truly unsuitable for the development of $C$. glomerata's offspring. In Chapter 6, I tested whether $M$. brassicae was indeed a non-host for $C$. glomerata, by verifying egg deposition and egg survival. I expected that these eggs would not survive and that $C$. glomerata would alter its behaviour after such a non-host oviposition experience. An expectation that was in line with my third hypothesis. I used a couple of behaviour assays to test for this change in behaviour, including the high-throughput individual T-maze from chapter 2 , and addressed the question why $C$. glomerata deposits eggs in M. brassicae.

Finally, in Chapter 7, I integrated the findings of previous chapters and synthesized how parasitoid foraging behaviour is shaped by information-reliability and non-host interactions. I addressed the hypotheses stated above and presented an overview of methods to measure parasitoid foraging behaviour in the laboratory and the field. Furthermore, I discussed the context-dependency of parasitoid foraging behaviour, how parasitoid learning can benefit biological control efforts and I propose future research direction.

\section{Acknowledgments}

I thank Jetske de Boer, Hans Smid and Louise Vet for their constructive comments on a previous version of this chapter. 




\title{
Chapter 2
}

\section{Automated high-throughput individual tracking system for insect behaviour: Applications on memory retention in parasitoid wasps}

\author{
Jessica A.C. de Bruijn ${ }^{1}$ \\ Louise E.M. Vet ${ }^{1,2}$ \\ Maarten A. Jongsma ${ }^{3}$ \\ Hans M. Smid ${ }^{1}$
}

${ }^{\text {a }}$ Laboratory of Entomology, Plant Sciences Group, Wageningen University, Wageningen, The Netherlands

${ }^{b}$ Department of Terrestrial Ecology, Netherlands Institute of Ecology (NIOO-KNAW), Wageningen, The Netherlands

${ }^{\mathrm{c}}$ Plant Research International, Wageningen University, Wageningen, The Netherlands

Published in a slightly different form in Journal of Neuroscience Methods (2018) 309: 208-217 


\begin{abstract}
Insects are important models to study learning and memory formation in both an ecological and neuroscience context due to their small size, behavioural flexibility and ecological diversity. Measuring memory retention is often done through simple time-consuming set-ups, producing only a single parameter for conditioned behaviour. In this study, we present the design of the High-throughput individual Tmaze and its use for testing memory retention in two very different parasitoid species, the larval parasitoid Cotesia glomerata and the pupal parasitoid Nasonia vitripennis. The high-throughput individual T-maze uses commercially available tracking software, Ethovision $\mathrm{XT}^{\circledR}$, in combination with a Perspex stack of plates as small as $18 \times 18 \mathrm{~cm}$, which accommodates 36 olfactory T-mazes, where each individual parasitoid can choose between two artificial odours. Various behavioural parameters, relevant to memory retention, can be acquired with this set-up, such as first choice, residence time, giving up time and zone entries. From these parameters, a performance index can be calculated as a measure of memory retention. Groups of 36 parasitoids can be tested simultaneously within minutes, resulting in efficient acquisition of sufficiently high sample sizes. The set-up, and its corresponding methods, proved to be highly suitable for testing memory retention in both $C$. glomerata and $N$. vitripennis. The high-throughput individual T-maze provides us with a standardized high-throughput, labour-efficient and cost-effective method to test various kinds of behaviour, offering excellent opportunities for comparative studies of various aspects of insect behaviour.
\end{abstract}

Keywords: learning, memory retention, parasitoid wasps, Cotesia glomerata, Nasonia vitripennis, tracking system 


\section{Introduction}

Learning and memory formation are universal traits in the Animal Kingdom (Dubnau, 2003), which makes it possible to study them in a wide range of animal species with varying levels of brain complexity, including insects, such as fruit flies (Drosophila melanogaster), bees (Apis melifera) and parasitoid wasps (Chen and Tonegawa, 1997; Margulies et al., 2005; Smid et al., 2007; Galizia et al., 2011). For ecological and neuroscience studies insects are ideal models due to their small size, behavioural flexibility and enormous ecological diversity.

Memory retention is an important parameter in studies of learning and memory formation, and it is generally assessed by measuring conditioned behaviour. Many different bioassays have been used to study memory retention in insects, such as the proboscis extension reflex (Bitterman et al., 1983), the two-choice wind tunnel (Geervliet et al., 1998b), the Y-tube olfactometer (Wäckers, 1994), the static twochamber olfactometer (Huigens et al., 2009), the four-quadrant olfactometer (Vet et al., 1983), the locomotion compensator (servosphere) (Vet and Papaj, 1992) and the T-maze olfactometer (Hoedjes et al., 2012; Jiang et al., 2016). These bioassays measure memory retention through conditioned behaviour in different ways and each has its own strengths and weaknesses. The two-choice wind tunnel, the four-quadrant olfactometer and servosphere bioassays allow for detailed recording of biologically relevant behavioural responses of individual insects, but are time consuming. Wind tunnels also require expensive equipment and ample space. The T-maze olfactometer is used with groups of insects, which is more time efficient, but data points are formed per group and therefore many conditioned animals are required per experiment to obtain sufficient sample sizes. Furthermore, information on different parameters of individual behaviour are not recorded (Lin et al., 2015) and social behaviour may affect the observed behavioural response (Kohn et al., 2013).

A bioassay consisting of a video set-up with automated tracking software and a welldesigned test system can solve several of the above described drawbacks. Automated tracking software allows for detailed recording of many behavioural parameters and has already been used in several studies, but generally only with recordings of a single individual or with group release where individual identities are lost (Faucher et al., 2006; Beshel and Zhong, 2013; Reza et al., 2013; Spitzen et al., 2013; Smith and Raine, 2014; Lin et al., 2015). Recently, further technological advancements in studies on insect behaviour have been realized with video tracking software, where the behaviour of individual insects in multiple arenas can be simultaneously recorded 
and tracked, allowing for both detailed individual behavioural recording and highthroughput (Kloth et al., 2015; Thoen et al., 2016).

In this study a novel bio-assay was designed for memory retention testing in parasitoid wasps. This set-up consists of a block with 36 individual olfactory T-maze arenas in combination with a video set-up and tracking software, and allows for simultaneous automated behavioural tracking of 36 individual parasitoids. We used complex, commercially available odour extracts and compared the sensitivity of the parasitoids for these odours using the electro-antennogram technique. To test this novel bioassay, we used two unrelated and ecologically different parasitoid species, Cotesia glomerata and Nasonia vitripennis. Cotesia glomerata (Braconidae: Microgastrinae) is a parasitoid wasp that lays her eggs in first instar caterpillars of Pieridae butterflies. It forms long term memory (LTM) for specific host plant odours when they are rewarded with an oviposition in a caterpillar of the large cabbage white butterfly, Pieris brassicae, on that plant (Smid et al., 2007). The jewel wasp Nasonia vitripennis (Hymenoptera: Pteromalidae) lays her eggs in pupae of several fly species. It forms LTM for natural odour extracts after a single oviposition experience in a pupa of the bluebottle blowfly, Calliphora vomitoria (Hoedjes and Smid, 2014). To optimize the bioassay for use with these species, sensitivity, preference and memory retention experiments were conducted. The combined results suggest this system can be used for a broad range of parasitoid wasp species and may be further extended to include many more insect species and research fields.

\section{Materials and methods}

\section{Insect cultures}

Cotesia glomerata (Hymenoptera: Braconidae) females were obtained from a colony which is re-established each year from individuals collected from cabbage fields around Wageningen, The Netherlands. Parasitoids were reared on Pieris brassicae L. (Lepidoptera: Pieridae) caterpillars, which in turn were reared on cabbage plants (Brassicae oleracea) as described in Geervliet et al. (1998a). Parasitoid cocoons from this rearing were placed in cages $(40 \times 30 \times 30 \mathrm{~cm})$ in a climate chamber $(20$ $22^{\circ} \mathrm{C}, 50-70 \%$ relative humidity, photoperiod L16:D8) where parasitoids were supplied with honey and water. From these cages, two-day-old female parasitoids were collected and placed in a separate cage with water and honey until experiments started. Female parasitoids of 3-5 days old were used in all experiments. 
Nasonia vitripennis parasitoids (Hymenoptera: Pteromalidae, strain AsymCx) were reared as described in Hoedjes et al. (2012), in polystyrene rearing vials (28.5 x 95 $\mathrm{mm}$ ) with foam stoppers (Genesee Scientific, San Diego, CA, USA). Parasitoids were kept in a climate chamber at $25^{\circ} \mathrm{C}, 50-70 \%$ relative humidity, with a photoperiod of L16:D8. Pupae of the fly species Calliphora vomitoria (Kreikamp, Hoevelaken, the Netherlands) were used as host, as described in Hoedjes et al. (2012). Female parasitoids were fed honey and water and were used 2-4 days after emergence.

\section{Odours used for conditioning and memory retention testing}

Four different commercially available complex odour blends were used: 2x Royal Brand bourbon Vanilla extract, Natural Chocolate extract, Pure Coffee extract, and Natural Almond extract (Nielsen-Massay Vanillas Intl., Leeuwarden, the Netherlands). The choice for these odours was based on earlier studies on learning and memory formation in Nasonia parasitoids (e.g. Hoedjes et al, (2012), (2014), (2015), Liefting et al. (2018), van der Woude et al. (2018)). These blends were chosen, since they were not expected to evoke high innate responses in the tested parasitoid species, as they are not present at host or food sites. Furthermore, since they are composed of many different odorants, they are also unlikely to remain undetected. Odour detection was previously confirmed for $N$. vitripennis using electroantennogram (EAG) analysis (Hoedjes et al., 2012), showing that at the antennal level, these odours showed doses-dependent responses.

For C. glomerata, such EAG experiments were performed in this study. For behavioural bioassays, concentrations of these odours could be fine-tuned to obtain a $50 \%-50 \%$ choice from unexperienced parasitoids in a T-maze and clear-cut conditioned responses to each side of the T-maze (Hoedjes et al., 2012). The additional advantage of using odour blends, which are unrelated to the biology of the parasitoids, is that such odours provide the best opportunity to get unbiased results in memory studies, where different species are compared. 


\section{Electroantennogram analysis for $C$. glomerata}

An electroantennogram (EAG) analysis was conducted to assess the sensitivity to several complex natural odour blends at the level of the olfactory sensilla on the antenna, because potential differences could affect the detection of memory retention in subsequent experiments. The EAG set-up was adapted from Hoedjes et al. (2012), and based on a commercially available set-up from Syntech, Hilversum, The Netherlands. We performed EAG analysis with commercially available odour blends. The odour extracts were dissolved in a $50 \mathrm{ml} 4 \%$ agarose (A9539-500g, Sigma) solution in deionized water, at odour concentrations of $1 \%, 4 \%, 16 \%$ and $64 \%$. Odour blends were heated to $80^{\circ} \mathrm{C}$ in a water bath and were then added to the agarose solution at the same temperature, and mixed with a magnetic stirrer. The control agarose solution was made without odour extract. Solutions were poured on a flat plastic sheet (OHP Transparency film, Nobo ACCO Brands Cooperation, England). The agarose was allowed to spread out on the sheet, to level out and dry for 30 minutes. Strips of $40 \times 5 \times 2 \mathrm{~mm}$ agarose were cut from the centre of the dried agarose solutions and a strip was placed against the inner wall of a Pasteur pipette, where it would not block the airflow. Pasteur pipettes were subsequently sealed with parafilm until the start of the EAG analysis. Just before the start of the experiment the Pasteur pipettes were flushed with $250 \mathrm{ml}$ of clean air to standardize odour release. Unconditioned $C$. glomerata females were anaesthetized by putting them briefly on ice, after which they were decapitated and the last segment of one of the antennae was cut off. The base of the head was connected to the ground electrode of the EAG set-up and the cut antenna to the recording electrode. We used $4 \%$ almond as a standard odour and corrected with the unscented control agarose to calculate relative EAG responses as described in Hoedjes et al. (2012).

\section{Conditioning procedures}

\section{$\underline{\text { Cotesia glomerata }}$}

Female parasitoids were given an associative learning experience using a classical conditioning procedure, adapted from Bleeker et al. (2006). In the original procedure, parasitoids learned to associate plant odours as the conditioned stimulus (CS) with suitable hosts as the unconditioned stimulus (US), after a single oviposition experience with a caterpillar on a plant leaf. This type of conditioning is considered a form of classical (Pavlovian) conditioning, where the host-searching 
phase is excluded. Smid et al. (2007) later found that his single trial conditioning method resulted in the formation of robust, protein synthesis-dependent long-term memory (LTM). In the current study, odourised agarose was used as CS, instead of plant leaves, on which caterpillars and so-called frass (faeces and silk produced by the feeding caterpillars) were placed. The agarose odourised with vanilla or coffee extract was made as described above at a $4 \%$ odour concentration. A globular sphere of odourised agarose was made by dipping the cone of a micro pestle (SIAL501ZZ0, Sigma-Aldrich) 5 times in the odourised agarose solution at intervals of 30 seconds, resulting in a globular shaped substrate $(15 \mathrm{~mm}$ diameter) on a stick. The agarose was allowed to cool at room temperature for 30 minutes after which 150-200 first instar caterpillars and frass were placed on top of the agarose.

Two hours before conditioning, parasitoids were retrieved from the climate cabinet and placed in the laboratory to acclimatize. For conditioning, 15 parasitoids were individually transferred to polystyrene rearing vials and sequentially offered the odourised agarose stick with the caterpillars to allow each parasitoid a single oviposition in a host. Upon offering the stick with odourised agarose, caterpillars and frass, parasitoids immediately initiated oviposition and a single oviposition was generally completed within 30 seconds. After ovipositing, parasitoids were captured by letting them walk into a clean vial. The parasitoids were then transferred to a small cage (Dimensions $17.5 \times 17.5 \times 17.5 \mathrm{~cm}$, Bugdorm type 41515, Megaview Science, Taiwan) with water and honey and kept in a climate chamber until testing $24 \mathrm{~h}$ later. Parasitoids were reciprocally conditioned with two different odours for memory retention experiments: 15 parasitoids were given an oviposition experience on vanilla scented agarose and 15 parasitoids on coffee scented agarose.

\section{Nasonia vitripennis}

Conditioning trials for $N$. vitripennis were done as described in Hoedjes et al. (2012). This conditioning procedure is known to induce protein synthesis-dependent LTM in this strain (Hoedjes and Smid, 2014). Coffee and vanilla extracts were used to train and test parasitoids. Parasitoids were given an associative learning experience with a reciprocal, differential classical conditioning procedure, where half of a group of parasitoids was first given an associative learning experience with vanilla odour and a host (CS+), after which it was exposed to coffee odour without a host (CS-). The other reciprocal half of the group was conditioned with the same odours, but in opposite order, so coffee odour as CS+ and vanilla odour as CS-. The associative 
learning experience was conducted by placing parasitoids individually in a well of a 12-well microtiter plate (Greiner Bio-One, Alphen aan den Rijn, the Netherlands), each well containing two Calliphora vomitoria pupae and a piece of filter paper $(0.75$ $\mathrm{cm}^{2}$ ) with $1 \mu \mathrm{l}$ pure vanilla or coffee extract. During a $1 \mathrm{~h}$ period the parasitoid would drill and host feed while experiencing the odour to form the association. A group of 12 parasitoids was given this experience individually. Actual oviposition does not take place with this conditioning protocol, but previous experiments have shown that deposition of eggs in not required to form LTM (Hoedjes and Smid, 2014). Parasitoids that did not start drilling within the first 30 minutes of conditioning were excluded from experiments. After the CS+ experience, parasitoids were transferred to a polystyrene vial for 15 minutes. They were then exposed to the CS- for 15 minutes. Here, the odour was offered in the form of a glass capillary (ID $1.3 \mathrm{~mm}$, cut to $30 \mathrm{~mm}$; Fisher Emergo, Landsmeer, the Netherlands) filled with pure odour extract and covered at one end with pure petroleum jelly (Vaseline original, Unilever Nederland B.V., Rotterdam). This entire procedure was defined as one single conditioning trial and lasted 90 minutes. This difference in duration of a conditioning trial with $C$. glomerata reflects the difference in duration of the oviposition behaviour between the two species. After conditioning, parasitoids were transferred to a clean polystyrene vial with honey and water and placed back in the climate chamber until testing the following day.

\section{Memory retention bioassays}

\section{High-throughput Individual T-maze design}

The high-throughput individual T-maze design is based on the video tracking set-up described in Thoen et al. (2016) and Thoen (2016) for thrips, which is here redesigned for use with parasitoid wasps. The system consisted of a stack of five Perspex plates with dimensions of $180 \times 180 \mathrm{~mm}$ and thickness of 2, 5 or $10 \mathrm{~mm}$ (PyraSied BV., Leeuwarden, The Netherlands). In these plates different openings were made, using a computer guided laser cutting machine (BRM 6090 Lasermachine, BRM Lasers, Winterswijk, the Netherlands). Together, they formed 36 T-maze arenas for individual testing of 36 parasitoids simultaneously (Fig. 1).

The different layers of transparent Perspex plates were divided into two compartments (Fig. 1). The bottom compartment served as 36 cages to load and hold 36 parasitoids (Fig. 1b) until their release at the start of the experiment, whereas the 
top compartment consisted of the actual T-maze arenas situated directly above each of the 36 cages. The bottom compartment could be closed or opened towards the top compartment by a gate plate, to allow for simultaneous release of parasitoids from the cages into the T-maze arenas (Fig. 1b, c).

The bottom compartment with the cages and the gate was formed by four layers, from top to bottom: one gate plate $(195 \times 180 \times 2 \mathrm{~mm})$ with 36 circular holes of $5 \mathrm{~mm}$ diameter (Fig. 1f). By sliding this plate back- or forwards, the holes in this plate could be aligned (Fig. 1c) or closed (Fig 1b), thereby opening or closing the connection between the cages and arenas. The second plate $(180 \times 180 \times 10 \mathrm{~mm})$ formed the actual cages, with $365 \mathrm{~mm}$ cylindrical openings where parasitoids were trapped until testing commenced (Fig. 1g).

Below this second layer was a third layer, the bottom sliding door plate, which consisted of four slides $(180 \times 41 \times 5 \mathrm{~mm})$ which could move on a Perspex plate of $180 \times 180 \times 2 \mathrm{~mm}$ (Fig. 1h). To allow free movement of these slides, the bottom plate had two $180 \times 5 \times 5 \mathrm{~mm}$ Perspex pieces glued on the left and right sides and $180 \times 2 \times 5 \mathrm{~mm}$ spacers glued between individual slides. In the centre of the slide opening of the bottom plate, four longitudinal slits of $160 \times 10 \mathrm{~mm}$ were made to allow access to the slides from the bottom. Each slide had nine holes, positioned directly underneath the cage cells, and were covered on the top with gauze (Monodur, PA 250; Nedfilter b.v., Almere, the Netherlands) for bottom ventilation of the cells. The slides allow for opening (Fig 1b, left) and closing (Fig. 1b, right) of each consecutive cell by sliding them backwards or forward while loading parasitoids from below directly into the cage cells thereby using the natural, negative geotaxis of the parasitoids.

Above the bottom compartment (cage and gate) is the top compartment, which consisted of the arena plate and the top plate. The arena plate $(180 \times 180 \times 10 \mathrm{~mm})$ consisted of 36 two-choice arenas (Fig. 1e). Each arena was made of two circular lateral zones of $15 \mathrm{~mm}$ across and $8 \mathrm{~mm}$ deep, connected by a bridge $(10 \times 8 \times 5$ $\mathrm{mm}$ ) (Fig. 1a, c). The bridge is $3 \mathrm{~mm}$ higher than the lateral zones so that each lateral zone could be filled with a $3 \mathrm{~mm}$ (odourised) agarose layer. After application of the agarose layer, the bridge and lateral zones are at equal level (Fig. 1a). In the middle of the bridge, at equal distance to each of the lateral zones, a $5 \times 5 \mathrm{~mm}$ circular opening was made in line with the cages to allow parasitoids to enter the arena, when the gate is aligned with that opening (Fig. 1c). 
Chapter 2

a Single T-maze, frontal view

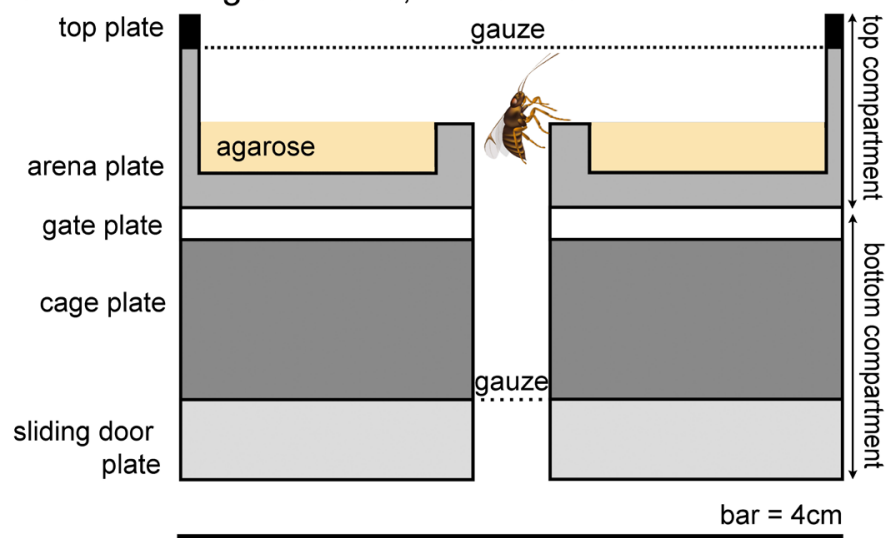

b Wasp loading, gate closed

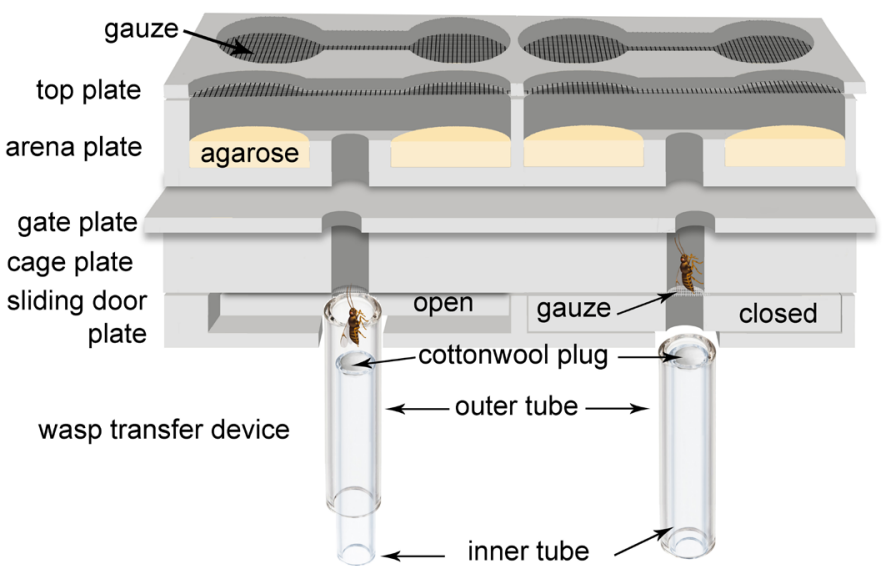

C Memory testing, gate open (drawing without top plate)

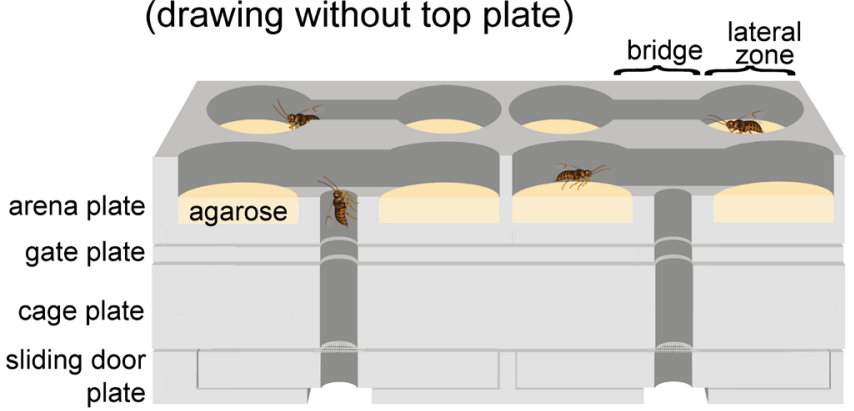

d Top plate

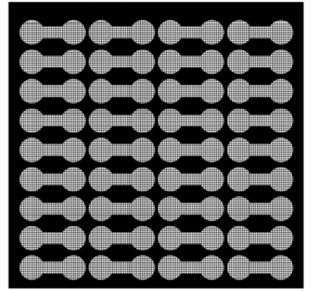

e Arena plate

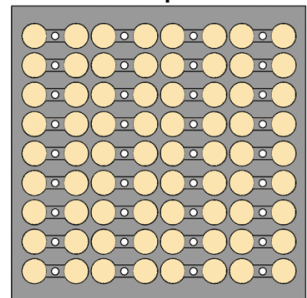

f Gate plate

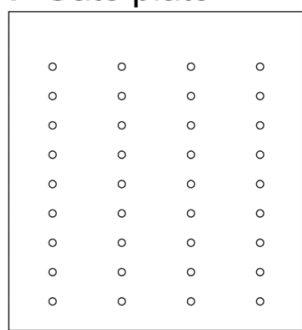

\section{g Cage plate}

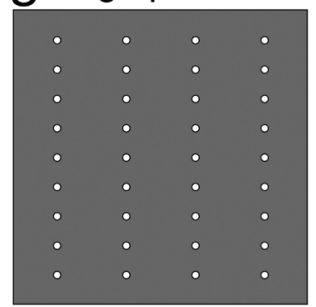

h Sliding door plate

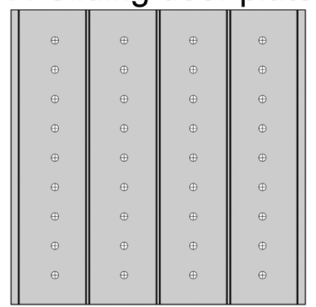


Figure 1. Drawings of the T-maze set up. All elements are labelled in the figures. One T-maze consisted of multiple layers of Perspex plates. The bottom compartment consisted of the bottom sliding door plate and the cage plate. Above the bottom compartment was the gate plate. The top compartment consisted of the arena plate with the agarose zones and the top plate (a) Cross section of one single T-maze, frontal view, out of the complete block of 36. This drawing has been made to scale (scale bar $=4 \mathrm{~cm}$ ). The odourised agarose is coloured yellow, the other elements have different shades of grey to distinguish them, but all plates are of the same transparent Perspex material. (b) Drawing in perspective of a set of 4 arena's and connected cages, in parasitoid loading position, with the gate closed. The left row has the bottom sliding door opened, and a parasitoid can be loaded from the bottom using the parasitoid transfer device. The right row has the bottom sliding door closed, and the parasitoid is in the cage compartment. (c) After loading of all parasitoids, the gate is opened allowing the parasitoids to enter the arenas from their cages and start exploring the two fields of odourised agarose. (d-h) The 5 different plates of Perspex that together form the block with $36 \mathrm{~T}$-mazes, from top to bottom. The grey shades correspond to those used in fig 1a.

Furthermore, the central circular opening in the bridge of the arena was $6 \mathrm{~mm}$ instead of $5 \mathrm{~mm}$ (Fig. 1d). The design of the arenas was exactly the same. The system was closed with a top plate $(180 \times 180 \times 2 \mathrm{~mm})$ where the area above each arena was cut out and covered with gauze for ventilation (Fig. 1d). The stack with all plates was aligned and kept together in a holder with an opening of $180 \times 180 \times 24 \mathrm{~mm}$ to prevent movement of plates and ensure exact alignment of the $5 \mathrm{~mm}$ openings of the cage, gate and central opening of the arenas through which parasitoids could walk.

For $N$. vitripennis a prior model of the high-throughput individual T-maze was used, where the top plate (Fig. 1d) had no opening for ventilation, where parasitoids were loaded from the top into the bottom compartment (the cage, Fig. 1b) instead of from the bottom and only 32 instead of 36 could be loaded in the system.

\section{Use of the High-throughput Individual T-maze}

Before experiments, odourised agarose solutions were prepared and $0.5 \mathrm{ml}$ was pipetted into the lateral zones of each arena after which it was left to dry at room temperature for 30 minutes. Odourised agarose was prepared with either vanilla, chocolate or coffee extract at different concentrations $(0.5,1,2$ and $4 \%)$ or control agarose, where no odour was dissolved in the agarose. Combinations of two odour pairs in different concentrations were used according to results obtained with unexperienced and experienced parasitoids as described in sections 3.2 and 3.3. The lateral zones of each arena were always filled with two different odour solutions to present a two-choice situation. The location of a specific odour was alternated in every other arena. Once the agarose had dried, 36 parasitoids were taken from their cage using a transfer device (Fig. 1b). This transfer device consisted of an outer glass 
tube (outer diameter $8 \mathrm{~mm}$, inner diameter $6 \mathrm{~mm}$, length $6 \mathrm{~cm}$ ) in which an inner tube capped with cotton wool was placed (outer diameter $5 \mathrm{~mm}$, inner diameter $4 \mathrm{~mm}$ length $6.5 \mathrm{~cm}$ ). With this device parasitoids could gently be pushed forward out of the transfer device and loaded into the bottom compartment. Hereafter the highthroughput individual T-maze was placed underneath the camera set-up. Upon opening of the gate of the system, to allow the simultaneous release of the parasitoids into the two-choice arenas, behaviour was recorded for 10 minutes. Per recording 36 C. glomerata parasitoids, 12 parasitoids per treatment, were tested. For $N$. vitripennis groups of 29-32 vanilla or coffee conditioned parasitoids were tested.

All experiments were repeated on at least three different days, and treatment groups were loaded in a single plate in a randomized block design for $C$. glomerata. After testing, agarose was removed and plates were cleaned with soap (Bosmanite AL-42, Rogier Bosman Chemie B.V., Dinteloord, the Netherlands) and warm water.

\section{Camera set-up}

The complete high-throughput individual T-maze was placed on a backlight (FL tubes, $5000 \mathrm{~K}$ ) on $15 \mathrm{~mm}$ spacers, in a camera set-up (Fig. 2), which consisted of a digital camera (GigE, Basler acA2040-25-gc) with a varifocal lens (Kowa LM35HC 1" 35mm F1.4 manual iris c-mount). The entire set-up was shielded from daylight during recording by a black curtain with a white inner liner facing the set-up. Behaviour in the high-throughput individual T-maze was recorded using Debut Video Capture Software (v 1.88, ( NCH Software) at a 2046 x 2046 pixel resolution, a frame rate of $12.76 \mathrm{fps}$ and .mp4 file format. 


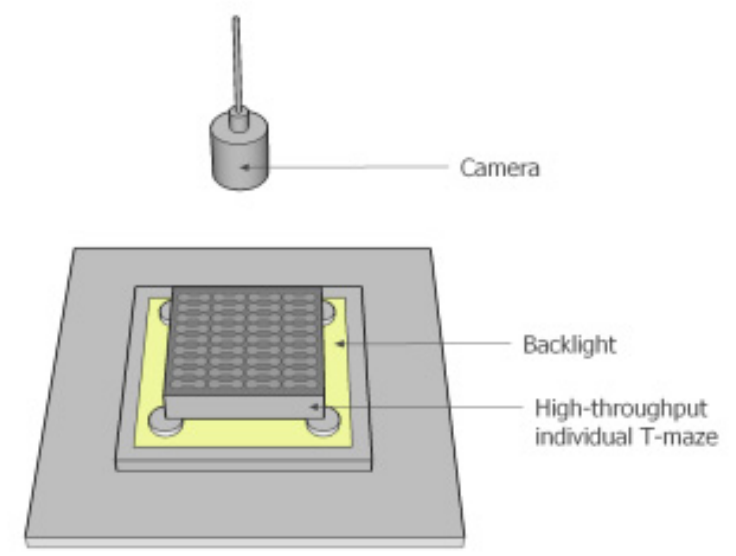

Figure 2. The camera set-up with the high-throughput individual T-maze in place. The high-throughput individual T-maze is placed on top of a backlight. The camera was positioned directly above the centre of the bioassay for an optimal view of all arenas.

\section{$\underline{\text { Video analysis }}$}

Video recordings were analysed with EthoVision ${ }^{\circledR}$ XT version 11.5 (Noldus Information Technology B.V., Wageningen, The Netherlands). Each arena was defined in EthoVision as consisting of 3 zones, two lateral zones in which the two odour sources were present, and a neutral zone, which consisted of the bridge and entry hole. Walking behaviour of the individual parasitoids was tracked using Ethovison's differencing method at a detection sensitivity value of 13. Parasitoids were not tracked when in the bridge zone or when their velocity dropped below 0.21 $\mathrm{cm} / \mathrm{s}$, and tracking started again above $0.25 \mathrm{~cm} / \mathrm{s}$. Tracking started once a parasitoid entered one of the lateral zones and paused when the parasitoid either stopped moving, or when it was present in the neutral zone. Behaviour was recorded until the total time spent moving in the lateral zones accumulated to 30 seconds. From the Ethovision ${ }^{\circledR}$ XT data output the following behavioural parameters were used; latency until first zone entry, latency until first zone exit (zone alteration), residence time and frequency of zone entry. Latency until first zone entry consisted of the time from parasitoid release, till its first entry in the lateral zone. Latency until first zone exit, defined as zone alteration in Ethovision ${ }^{\circledR} \mathrm{XT}$, consisted of the time from parasitoid release till the first time it exited a lateral zone. Residence time was defined as the total time a parasitoid spent moving in a lateral zone. Frequency of zone entry consisted of the number of times a parasitoid entered a lateral zone in the total recorded time. With this data we created the behavioural parameters first choice and 
giving-up time. First choice was determined by selecting the zone with the lowest latency until first zone entry. Giving-up time was determined by subtracting latency until first zone entry from latency until first zone exit (zone alteration). Residence time and zone entries (frequency of zone entry data) were used directly from Ethovision ${ }^{\circledR}$ XT. Parasitoids that did enter a lateral zone, but did not have 30 seconds of movement in the lateral zones in the 10 minutes recording, were only included in the analysis of first choice data. Their data for the other parameters was discarded.

\section{T-maze for group testing}

In order to compare the results obtained from high-throughput individual T-maze for memory retention in $N$. vitripennis with the previously used T-maze for groups (Hoedjes et al., 2012), we compared the two methods, following the same protocol and set-up as used by Hoedjes et al. (2012). Briefly, the T-maze consisted of three Plexiglas tubes, a central tube with a small opening in which the parasitoids were introduced and two lateral tubes through which an airflow of $100 \mathrm{ml} / \mathrm{min}$ was blown towards the central tube, where it could leave the system through ventilation slits covered by gauze. Odour was provided by placing two capillaries filled with either pure vanilla or coffee odour extract, in the airflow lateral to each arm of the T-maze. Groups of 9-12 parasitoids were released in the central tube and after 10 minutes the final choice was recorded by counting the number of parasitoids in each lateral tube. Parasitoids that did not make a choice, by remaining in the central tube, were regarded as non-responding. A total of 12 groups was tested for memory retention, 6 groups with vanilla as CS+ and 6 with coffee as CS+. Note that the final choice behavioural parameter, which was obtained from this bio-assay, cannot directly be compared with the first choice parameter measured in the high-throughput individual T-maze, since we only used the choice after 10 minutes. Furthermore, the size dimensions of the T-maze for group testing are much larger, and as a consequence, parasitoids are expected to switch between the two odours at a much lower frequency than in the high-throughput individual T-maze. Thus, final choice in the T-maze for group testing, as recorded after 10 minutes, may not necessarily be the first choice, but rather results from both choice behaviour, residence time and patch leaving tendency in the two lateral tubes of the T-maze. 


\section{Statistics}

For $C$. glomerata the relative EAG responses were analysed by a two-way ANOVA using SPSS, version 23 (IBM, Armonk, NY, USA), to test for differences in EAG response between the four odours and for concentration effects. Normality and equal variance assumptions were checked with normality and residual plots, after which pairwise comparisons were made using a Tukey's LSD.

First choice results of the odour preference experiment were statistically analysed using a binomial test. For memory retention testing in the high-throughput individual T-maze, Performance index (PI) scores of all four behavioural parameters were based on two parasitoids, one CS1+ (conditioned with odour 1, vanilla) parasitoid and one CS2+ (conditioned with odour 2, coffee) parasitoid. Their scores were combined to form one PI, as described below. The two corresponding parasitoids that contribute to one PI score were tested in a two-choice arena at the same position in plates analysed directly after each other. No PI score was formed if one of the two parasitoids did not respond during recording. In case of the binomial first choice results, the PI was calculated for each parasitoid pair as 100 if both the CS1+ parasitoid would first enter the conditioned agarose zone with odour 1 and the CS2+ parasitoid first entered the zone with odour 2. If one of the two parasitoids entered the alternative zone first, then the PI would be 0 , if both would enter the alternative zone first, the PI was -100. No PI score was formed if one of the two parasitoids did not respond during recording. For giving up time and total residence time, PI scores were calculated per parasitoid pair by subtracting the percentage of active searching time that the $\mathrm{CS} 2+$ parasitoid spent on the CS1 zone from the percentage of time the $\mathrm{CS} 1+$ parasitoid spent on the CS1 zone (PI $=\%$ time CS1+ parasitoid on CS1 - \% time CS2+ parasitoid on CS1). The same was done for zone entry data, but here instead of the percentage of time, the percentage of visits to either zone was used (PI $=\%$ visits $\mathrm{CS} 1+$ parasitoid to $\mathrm{CS} 1-\%$ visits CS2+ parasitoid to CS1). All datasets from these calculations consisted of PI values ranging between -100 to 100, where a value of -100 represented a negative effect of conditioning, 0 represented no effect of conditioning and 100 a maximum effect of conditioning. More details on how these PI scores were calculated can be found in the supplementary information. Since not all datasets were normally distributed, all average PI scores were statistically analysed with a one-sample Wilcoxon's signed rank test, to test if their values where significantly higher than 0 , which would indicate memory retention (Hoedjes et al., 2012). 
For the T-maze for group testing, we used performance index (PI) scores for memory retention experiments as described in Hoedjes et al. (2012), with data of reciprocally tested groups. One group was given a conditioning trial in combination with odour 1 as CS (CS1+ parasitoids), the other with odour 2 as CS (CS2+ parasitoids). After testing, the percentage of $\mathrm{CS} 2+$ parasitoids that had chosen odour 1 was subtracted from the percentage of $\mathrm{CS} 1+$ parasitoids that had chosen odour 1 . These PI scores were also statistically analysed with a one-sample Wilcoxon's signed rank test. In all cases, we used an alpha value of 0.05 as cut-off for significance.

\section{Results}

\section{Electroantennogram recordings of $C$. glomerata}

EAG analysis (Fig. 3) showed a significant effect of odour, odour concentration and the interaction between odour and odour concentration (odour: $F=110.612, p<$ 0.001; concentration: $F=87.678, p<0.001$; odour x concentration: $F=24.273, p<$ $0.001)$. Pairwise comparisons show that parasitoids were more sensitive to almond than to any of the other odours (Tukey's LSD, $p<0.001$, table 1 ). Sensitivity to vanilla, chocolate and coffee was not different. Due to the high sensitivity to the almond extract, this odour was not selected for further experiments.

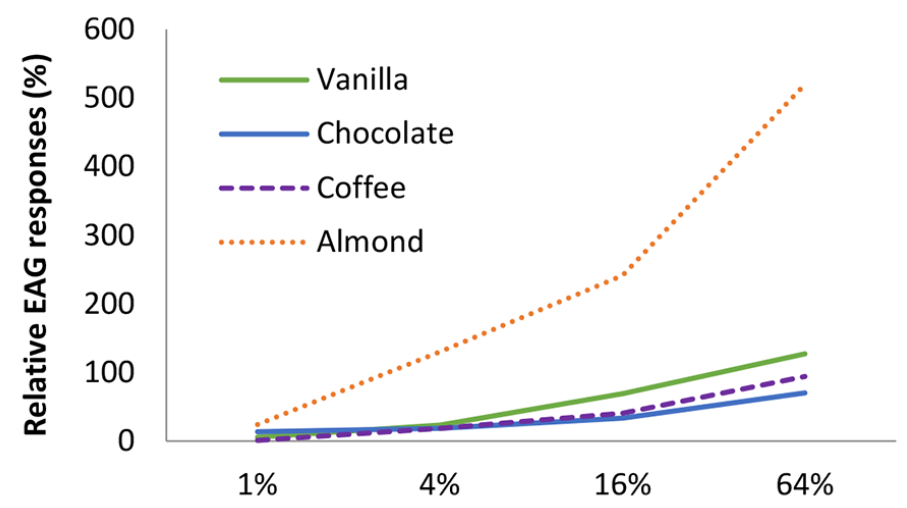

Figure 3. Relative EAG responses of C. glomerata with various concentrations of vanilla, chocolate, coffee and almond odours. Results were calculated by using $4 \%$ almond odour as a standard and by correcting with control odour results. There was a significant effect of both odour and concentration and their interaction, with sensitivity to almond being significantly different from vanilla, chocolate and coffee. 
Table 1. Pairwise comparison results of the different odours of the EAG of $C$. glomerata. Since a significant effect of odour was found in the EAG experiment with C. glomerata, the various odours were compared to find out which odours differed from each other.

\begin{tabular}{|l|l|l|}
\hline Odour 1 & Odour 2 & $\boldsymbol{p}$-value \\
\hline Almond & Chocolate & $<0.001$ \\
\hline & Coffee & $<0.001$ \\
\hline & Vanilla & $<0.001$ \\
\hline Chocolate & Coffee & 0.720 \\
\hline & Vanilla & 0.078 \\
\hline Coffee & Vanilla & 0.160 \\
\hline
\end{tabular}

\section{Odour preference of $C$. glomerata}

The selection of the odour pair for conditioning of $C$. glomerata was based on odour preference of unconditioned parasitoids (Fig. 4). First, we tested the preference for each type of $1 \%$ odourised agarose vs. unscented control agarose in the highthroughput individual T-maze. Three groups of 12 parasitoids were tested for each type of odourised agarose. First choice data showed that unconditioned parasitoids have an aversion to $1 \%$ chocolate $(\mathrm{F} 1=30 \%, p=0.043)$, whereas there was no preference for vanilla $(\mathrm{F} 1=46 \%, p=0.839)$ or coffee $(\mathrm{F} 1=42 \%, p=0.487)$ over the control agarose. Therefore, the chocolate extract was excluded from further testing. Combining the two remaining odours, and testing three groups of 36 unconditioned parasitoids with $1 \%$ vanilla vs. $1 \%$ coffee, showed no preference for either odour (Fig. 4, F1 vanilla $=47 \%, p=0.649$ ).

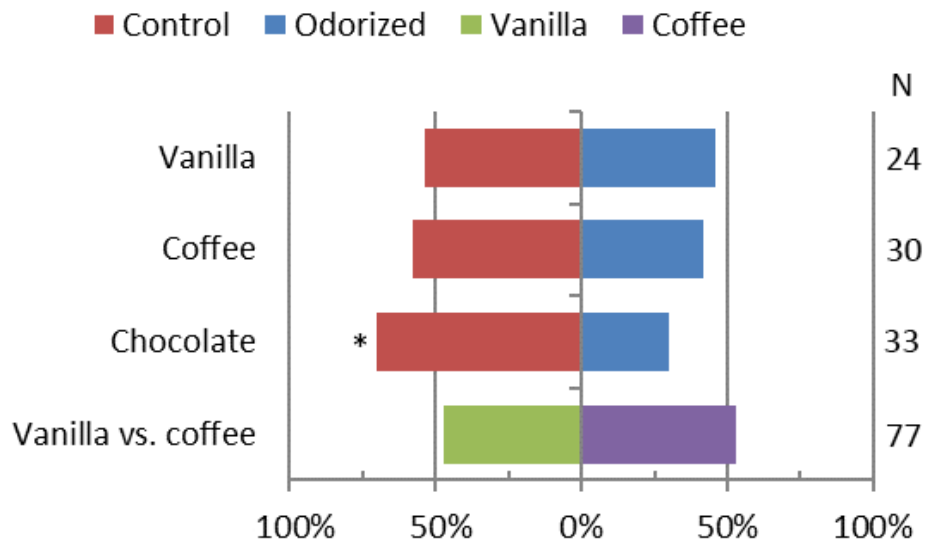

Figure 4. First choice results of unconditioned $C$. glomerata parasitoids with differently odorized agarose. Parasitoids were either tested with $1 \%$ odorized vs. control agarose, where vanilla, coffee and chocolate were the different odour options, or with $1 \%$ vanilla vs. $1 \%$ coffee agarose. 
Chapter 2

\section{Memory retention in $C$. glomerata}

Vanilla and coffee extracts were selected for conditioning (concentration 4\%) and memory retention testing (concentration 1\%) in accordance with above results. PI values of all tested parameters showed significant memory retention (Table 2, Fig. 5). Response levels of vanilla conditioned parasitoids were $68 \%(\mathrm{n}=23)$ and for coffee conditioned parasitoids $75 \%(\mathrm{n}=27)$. Underlying odour preference scores show a clear preference for vanilla with vanilla conditioned parasitoids, but no preference for coffee with coffee conditioned parasitoids (Table 2).

Since no preference was found for coffee with coffee conditioned parasitoids, different odour concentrations were tested to optimize the system; $1 \%$ vanilla vs. $0.5 \%$ coffee and $2 \%$ vanilla vs. $1 \%$ coffee. Testing with $1 \%$ vanilla and $0.5 \%$ coffee improved the preference of coffee conditioned parasitoids, but at the expense of vanilla conditioned parasitoids (Table 2). Response levels of vanilla conditioned parasitoids were $78 \%(\mathrm{n}=28)$ and for coffee conditioned parasitoids $86 \%(\mathrm{n}=31)$. PI values of all except first choice dropped and the giving up time parameter was no longer significant (Table 2, Fig. 5). Testing with $2 \%$ vanilla and $1 \%$ coffee resulted in low P-values for both vanilla and coffee conditioned parasitoids, though not all significant (Table 2 , vanilla $67 \%$ with $n=24$, coffee $72 \%$ with $n=26$ ). PI scores, however, were high and significant for all behavioural parameters (Table 2, Fig. 5). 
Table 2. Memory retention results of $C$. glomerata for the different behavioural parameters in the highthroughput individual T-maze, given different test odour concentrations. A One-Sample Wilcoxon's Signed Rank Test was used to test memory retention. Vanilla and coffee conditioned odour preference results were analysed using a $X^{2}$ test for first choice and a Wilcoxon's Signed Ranks Test for other behavioural parameters. The number (n) of PI scores used in the analysis is represented in the table.

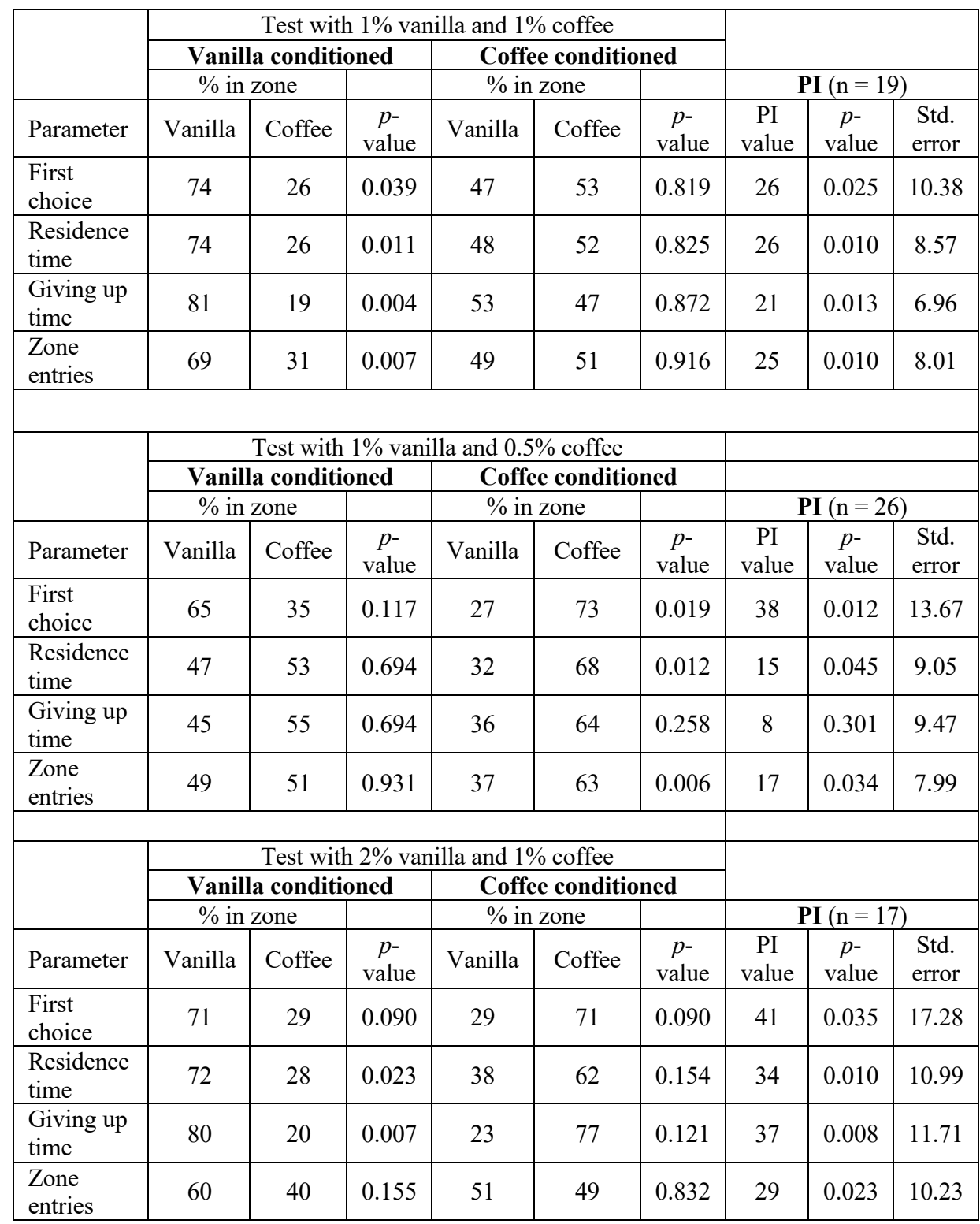


Table 3. Memory retention results of Nasonia vitripennis for the different behavioural parameters in the high-throughput individual T-maze and the T-maze for group testing. A One-Sample Wilcoxon's Signed Rank Test was used to test memory retention. Vanilla and coffee conditioned odour preference results were analysed using a $X^{2}$ test for first choice and final choice. For other behavioural parameters a Wilcoxon's Signed Ranks Test was used.

\begin{tabular}{|c|c|c|c|c|c|c|c|c|c|c|}
\hline \multirow[b]{5}{*}{ Parameter } & \multirow{2}{*}{\multicolumn{6}{|c|}{$\begin{array}{l}\text { High throughput individual T-maze } \\
\text { Test with } 1 \% \text { vanilla and } 1 \% \text { coffee agarose }\end{array}$}} & & & & \\
\hline & & & & & & & & & & \\
\hline & \multicolumn{3}{|c|}{ Vanilla conditioned } & \multicolumn{3}{|c|}{ Coffee conditioned } & & & & \\
\hline & \multicolumn{2}{|c|}{$\%$ in zone } & \multirow[b]{2}{*}{$\begin{array}{c}p- \\
\text { value }\end{array}$} & \multicolumn{2}{|c|}{$\%$ in zone } & \multirow[b]{2}{*}{$\begin{array}{c}p- \\
\text { value }\end{array}$} & \multicolumn{4}{|c|}{ PI } \\
\hline & Vanilla & Coffee & & Vanilla & Coffee & & $\begin{array}{c}\text { PI } \\
\text { value }\end{array}$ & $\begin{array}{c}p- \\
\text { value }\end{array}$ & $\begin{array}{l}\text { Std. } \\
\text { error }\end{array}$ & $\mathrm{n}$ \\
\hline $\begin{array}{c}\text { First } \\
\text { choice }\end{array}$ & 63 & 37 & 0.069 & 8 & 92 & $\begin{array}{c}< \\
0.001\end{array}$ & 55 & $<0.001$ & 8.07 & 51 \\
\hline $\begin{array}{l}\text { Residence } \\
\text { time }\end{array}$ & 71 & 29 & $\begin{array}{c}<< \\
0.001\end{array}$ & 15 & 85 & $\begin{array}{c}< \\
<.001\end{array}$ & 56 & $<0.001$ & 5.25 & 48 \\
\hline $\begin{array}{l}\text { Giving up } \\
\text { time }\end{array}$ & 75 & 25 & $\begin{array}{c}< \\
0.001\end{array}$ & 21 & 79 & $\begin{array}{c}< \\
0.001\end{array}$ & 52 & $<0.001$ & 6.27 & 48 \\
\hline \multirow[t]{5}{*}{$\begin{array}{c}\text { Zone } \\
\text { entries }\end{array}$} & 58 & 42 & 0.005 & 22 & 78 & $\begin{array}{c}< \\
0.001\end{array}$ & 45 & $<0.001$ & 5.29 & 48 \\
\hline & \multicolumn{6}{|c|}{ T-maze for group testing } & & & & \\
\hline & \multicolumn{6}{|c|}{ Test with vanilla and coffee extract capillaries } & & & & \\
\hline & \multicolumn{3}{|c|}{$\begin{array}{l}\text { Vanilla conditioned } \\
(\mathrm{n}=55)\end{array}$} & \multicolumn{3}{|c|}{$\begin{array}{c}\text { Coffee conditioned } \\
(\mathrm{n}=56)\end{array}$} & & & & \\
\hline & \multicolumn{2}{|c|}{$\%$ in zone } & & \multicolumn{2}{|c|}{$\%$ in zone } & & \multicolumn{4}{|c|}{ PI } \\
\hline Parameter & Vanilla & Coffee & $\begin{array}{c}p- \\
\text { value }\end{array}$ & Vanilla & Coffee & $\begin{array}{c}p- \\
\text { value }\end{array}$ & $\begin{array}{c}\text { PI } \\
\text { value }\end{array}$ & $\begin{array}{c}p- \\
\text { value }\end{array}$ & $\begin{array}{l}\text { Std. } \\
\text { error }\end{array}$ & $\mathrm{n}$ \\
\hline $\begin{array}{l}\text { Final } \\
\text { choice }\end{array}$ & 69 & 31 & 0.005 & 25 & 75 & $\begin{array}{c}<< \\
0.001\end{array}$ & 43 & 0.028 & 8.63 & 6 \\
\hline
\end{tabular}

\section{Memory retention in $N$. vitripennis}

Testing $N$. vitripennis in the high-throughput individual T-maze resulted in highly significant PI scores and significant results for almost all odour preference parameters (Table 3, Fig. 6). Testing in the T-maze for group testing resulted in a significant PI score for final choice (Table 3, Fig. 6). Response levels in the highthroughput individual T-maze ranged from $75 \%$ to $84 \%$, response levels of the Tmaze for group testing ranged from 80 to $81 \%$. 


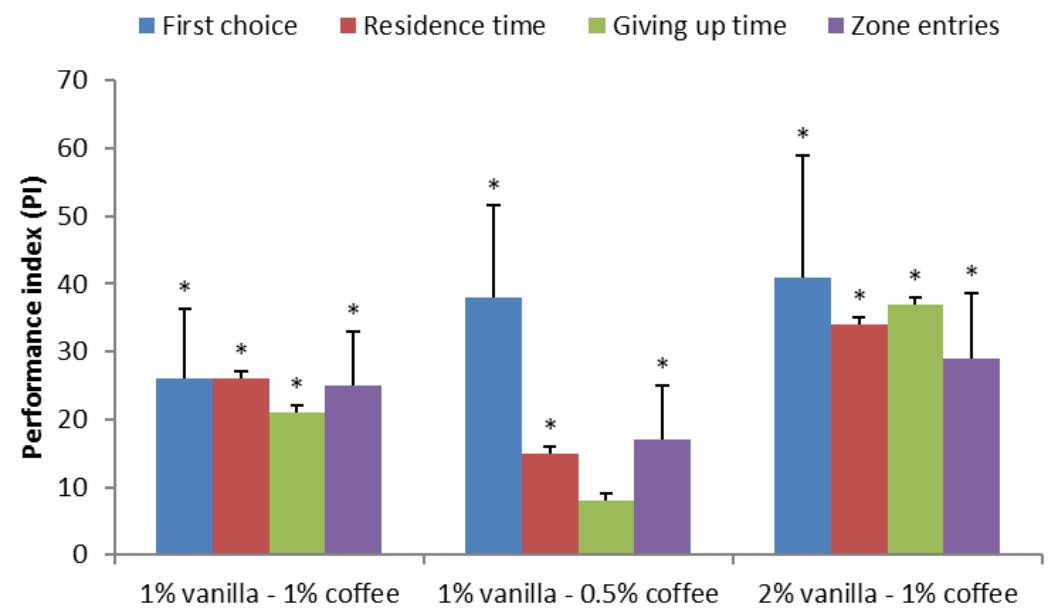

Figure 5. PI scores of the behavioural parameters of $C$. glomerata in the high-throughput individual Tmaze with different test odour concentrations. C. glomerata was tested in the high-throughput individual T-maze with test odour concentrations of $1 \%$ vanilla and $1 \%$ coffee $(n=19), 1 \%$ vanilla and $0.5 \%$ coffee $(n=26)$ and $2 \%$ vanilla and $1 \%$ coffee ( $\mathrm{n}$ first choice $=17$, other parameters $\mathrm{n}=16$ ). Significant PI scores $(p<0.05)$ are indicated with an asterisk $(*)$.

\section{घ High-throughput individual T-maze $\square$ T-maze for group testing}

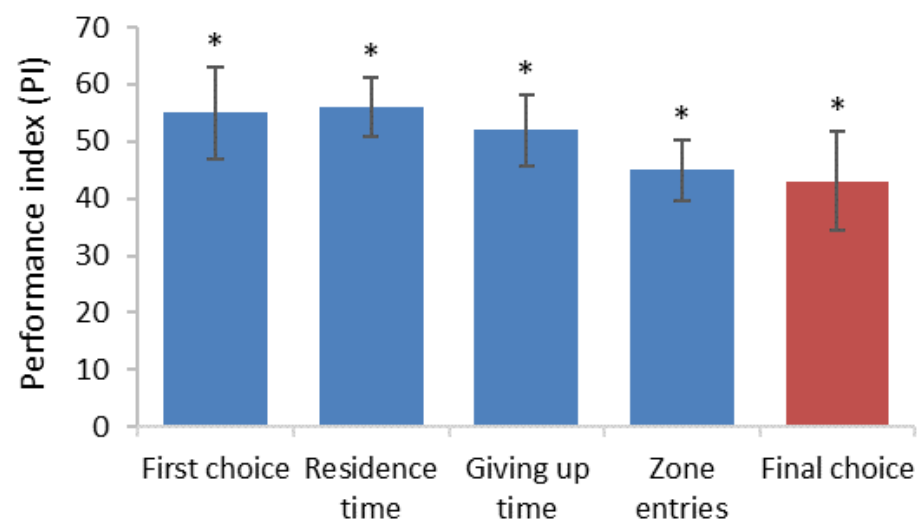

Figure 6. PI scores of the behavioural parameters of $N$. vitripennis in the high-throughput individual T-maze and the T-maze for group testing. $N$. vitripennis was tested with $1 \%$ vanilla and $1 \%$ coffee agarose in the high-throughput individual T-maze ( $\mathrm{n}$ first choice $=51$, other parameters $n=48$ ). With the T-maze for group testing only final choice could be assessed (Final choice $n=6$ ). Significant PI scores $(p<0.05)$ are indicated with an asterisk $(*)$. 


\section{Discussion}

Behavioural assays for insects have undergone a clear technological evolution in the past two decades. Time consuming methods, using observations of individual insects, have been redesigned with the latest advances in video tracking technology (Faucher et al., 2006; Beshel and Zhong, 2013; Reza et al., 2013; Smith and Burden, 2014; Lin et al., 2015; Jiang et al., 2016). Whereas various of these studies still test single insects (Faucher et al., 2006; Reza et al., 2013; Smith and Burden, 2014), our high-throughput individual T-maze makes it possible to load 36 parasitoids in individual cages from which they can simultaneously be released into their own twochoice arena. The camera set-up was combined with commercially available video software and multiple arena tracking software (Noldus et al., 2001), which allows for tracking of many individual parasitoids. Though simultaneous tracking of multiple insects in one arena has been reported previously (Beshel and Zhong, 2013; Lin et al., 2015; Jiang et al., 2016), individual identities of insects are often lost when walking tracks cross one another and social interactions may influence the results. The multiple arena tracking module of EthoVision makes it possible to assign many arenas in which individual parasitoids can be tracked. This allows for both highthroughput and recording of detailed individual behaviours, without social interactions and the need for massive amounts of insects. We showed that our system was able to detect multiple behavioural parameters suitable for measuring memory retention levels, thereby providing robust datasets in an efficient manner.

The conditioning and test protocols we used were designed to make them easy to standardize and reproduce with commercially available, natural odour blends. Our results emphasize that odour selection for conditioning and testing should be done carefully with both EAG and preference tests. Even though three natural odour extracts (vanilla, coffee and chocolate) showed an equal sensitivity in the EAG experiment, and are known to be used for conditioning parasitoid parasitoids (Lewis and Tumlinson, 1988; Lewis and Takasu, 1990; Gutiérrez-Ibáñez et al., 2007; Hoedjes et al., 2012; Zhou et al., 2015), our odour preference results of unconditioned parasitoids showed a clear aversion for the agarose odourised with chocolate vs. control agarose, whereas this was not the case for vanilla and coffee. An equal preference level of these odours to unconditioned parasitoids makes is easier to detect effects of conditioning. A final round of fine-tuning was performed by testing different concentration of odourised agarose in the individual T-maze. 
In order to find the best memory retention results it is important to assess if it is possible to induce a preference with each of the two odours used in the bioassay. Our results show that PI scores could be substantially increased when both odours showed significant conditioning effects. The reciprocal design of treatments eliminates any remaining odour bias and allows for the creation of performance index (PI) scores. These PI's are commonly used in studies on learning and memory formation as a parameter to measure conditioned behaviour, but usually these PI's are based on groups of insects (Hoedjes et al., 2012; Kohn et al., 2013; Jiang et al., 2016). With the development of a high-throughput individual T-maze, we were able to calculate PI scores based on two individual parasitoids, which increased sample sizes and therefore statistical power compared to PI's based on groups of insects. Robust PI scores based on each of 4 behavioural parameters for both C. glomerata and $N$. vitripennis were obtained in this study, demonstrating the suitability of this set-up for testing of memory retention. Using $N$. vitripennis, we compared the highthroughput individual T-maze with the T-maze for group testing, which showed comparable PI scores, but substantially better $p$-values, using a similar number of insects. This suggests that the required number of insects for the individual T-maze per experiment may be lower than for the T-maze for group testing

The high-throughput individual T-maze is a strong tool to advance knowledge of learning and memory dynamics in ecologically diverse groups such as parasitoid parasitoids. Results of $C$. glomerata and $N$. vitripennis show the system is likely to be suitable for a broader range of parasitoid wasp species and possibly also for other model insect species like D. melanogaster. Furthermore, due to the use of commercially available, natural odour blends, which are unrelated to odours parasitoids are exposed to in nature, it is possible to design comparative experiments with different species. Many types of preferences can be measured in this bioassay, such as food, colour and odour preferences, but also other types of behaviour such as mate choice and courtship behaviour, in line with what was done by Reza et al. (2013). The system allows for the selection of the most relevant and statistically strong behavioural parameters, allowing users to make species-specific selections to record various kinds of behaviours. Adaptations to the bioassay, to meet specific requirements of species, can be implemented easily, because of the flexibility of the laser-cutting methodology for manufacturing of the arenas and the low cost of the Perspex plates.

The selected behavioural parameters of the high-throughput individual T-maze; first choice, residence time, giving up time and zone entries, are all highly relevant for 
foraging success (Wajnberg, 2006). Although the conditions in the set-up described here are artificial, the fact that significant PIs were obtained from these 4 different behavioural parameters show that learning affects different aspects of foraging behaviour that contribute to foraging success for hosts, and thereby to realized fitness of the parasitoids. Our Cotesia model system provides excellent opportunities to validate how the results from our current high-throughput bio-assay translate into natural or agricultural situations, since $C$. glomerata is a well-known model species for behavioural studies in field, semi-field and wind tunnel situations (Geervliet et al., 1998a; Geervliet et al., 1998b; Benson et al., 2003; Bleeker et al., 2006; Smid et al., 2007; Kruidhof et al., 2012; Lucas-Barbosa et al., 2014; De Rijk et al., 2018). In addition, the set-up could be useful for efficient screening of relevant behavioural parameters of candidate species for biological control.

In conclusion, the high-throughput individual T-maze combines the benefits of highthroughput and individual testing. It provides us with a standardized highthroughput, labour-efficient and cost-effective method to test various kinds of behaviour and offers excellent opportunities for comparative studies of various aspects of insect behaviour.

\section{Acknowledgements}

This work was supported by the Netherlands Organisation for Scientific Research [grant number 824.14.023]. 


\section{Supplementary information}

\section{PI calculations}

For memory retention testing in the high-throughput individual T-maze, Performance index (PI) scores of all four behavioral parameters were based on one $\mathrm{CS} 1+$ (vanilla conditioned) parasitoid and one CS2+ (coffee conditioned) parasitoid. Their scores were combined to form one PI. The two corresponding parasitoids that contribute to one PI score were tested in a two-choice arena at the same position in plates analyzed directly after each other. No PI score was formed if one of the two parasitoids did not respond during recording.

\section{PI calculation for first choice data}

In case of the binomial first choice results, the PI was calculated for each parasitoid pair as 100 if both the $\mathrm{CS} 1+$ parasitoid would first enter the conditioned agarose zone with odor 1 and the CS2+ parasitoid first entered the zone with odor 2 . If one of the two entered the alternative zone first, then the PI would be 0 , if both would enter the alternative zone, the PI was -100 .

The formula of this calculation is:

$\mathrm{PI}=(\mathrm{CS} 1+$ zone choice $-\mathrm{CS} 2+$ zone choice $) * 100$

With the following assigned scores based on zone choice:

$\mathrm{CS} 1+$ choosing the CS1 zone is assigned a score of 1

$\mathrm{CS} 1+$ choosing the $\mathrm{CS} 2$ zone is assigned a score of 0

$\mathrm{CS} 2+$ choosing the $\mathrm{CS} 1$ zone is assigned a score of 1

$\mathrm{CS} 2+$ choosing the CS1 zone is assigned a score of 0

Leading to the following PI values for first choice data:

\begin{tabular}{|l|l|l|}
\hline CS1+ parasitoid & CS2+ parasitoid & PI calculation \\
\hline CS1 & CS2 & $(1-0)^{*} 100=100$ \\
\hline CS1 & CS1 & $(1-1)^{*} 100=0$ \\
\hline CS2 & CS2 & $(0-0)^{*} 100=0$ \\
\hline CS2 & CS1 & $(0-1)^{*} 100=-100$ \\
\hline
\end{tabular}


Chapter 2

\section{PI calculation for giving up time, total residence time and frequency of zone entry}

For giving up time, total residence time and frequency of zone entry, PI scores were calculated per parasitoid pair by subtracting the percentage of active searching time (or visits for frequency of zone entry) that the CS2+ parasitoid spent on the CS1 zone from the percentage of time the CS1+ parasitoid spent on CS1 zone.

First a percentage was calculated:

Giving up time

$\%$ time on CS1 $=($ time on CS1 $/($ time on CS1 + time on CS2 $)) * 100$

$\%$ time on $\mathrm{CS} 2=($ time on $\mathrm{CS} 2 /($ time on $\mathrm{CS} 1+$ time on CS2 $)) * 100$

Residence time

$\%$ time on CS1 $=($ time on CS1 / 30 $) * 100$

$\%$ time on $\mathrm{CS} 2=($ time on $\mathrm{CS} 2 / 30) * 100$

\section{Zone visits}

\% visits to $\mathrm{CS} 1=($ visits to $\mathrm{CS} 1 /($ visits to $\mathrm{CS} 1+$ visits to $\mathrm{CS} 2)) * 100$

$\%$ visits to $\mathrm{CS} 2=($ visits to $\mathrm{CS} 2 /($ visits to $\mathrm{CS} 1+$ visits to $\mathrm{CS} 2)) * 100$

All time units are in seconds, the division by 30 for residence time is based on the total residence time of 30 seconds.

Based on these percentages PI scores were calculated:

Giving up time (time based on the first visit only)

$\mathrm{PI}=\%$ time of a $\mathrm{CS} 1+$ parasitoid on $\mathrm{CS} 1-\%$ time of a $\mathrm{CS} 2+$ parasitoid on $\mathrm{CS} 1$

Total residence time

$\mathrm{PI}=\%$ time of a CS1 $1+$ parasitoid on $\mathrm{CS} 1-\%$ time of a $\mathrm{CS} 2+$ parasitoid on $\mathrm{CS} 1$

Frequency of zone entry

$\mathrm{PI}=\%$ visits of a CS1+ parasitoid to $\mathrm{CS} 1-\%$ visits of a $\mathrm{CS} 2+$ parasitoid to CS1 




\title{
Chapter 3
}

\section{Costs of persisting unreliable memory: Reduced foraging efficiency for free-flying parasitoid wasps in a wind tunnel}

\author{
Jessica A.C. de Bruijn ${ }^{1}$ \\ Louise E.M. Vet ${ }^{1,2}$ \\ Hans M. Smid ${ }^{1}$
}

${ }^{1}$ Laboratory of Entomology, Plant Sciences Group, Wageningen University, Wageningen, The Netherlands

${ }^{2}$ Department of Terrestrial Ecology, Netherlands Institute of Ecology (NIOO-KNAW), Wageningen, The Netherlands

Published in a slightly different form in Frontiers in Ecology and Evolution (2018) 6 


\begin{abstract}
Parasitoid wasps are known to improve their foraging efficiency after learning of herbivore-induced plant volatiles (HIPVs) upon encountering their hosts on these plants. However, due to spatial and temporal variation of herbivore communities, learned HIPV cues can become unreliable, no longer correctly predicting host presence. Little is known about the potential fitness costs when memories holding such unreliable information persist. Here we studied how persistent memory, containing unreliable information, affects the foraging efficiency for hosts in Cotesia glomerata. Parasitoids were conditioned to associate one of two types of HIPVs with either $P$. brassicae frass, 1 single oviposition in $P$. brassicae, 3 ovipositions in $P$. brassicae spaced in time or they were kept unconditioned. The following day, parasitoids were allowed to forage in a wind tunnel, in an environment that either conflicted or was congruent with their learned plant experience. The foraging environment consisted of host ( $P$. brassicae) and non-host (Mamestra brassicae) infested plants. The conflicting environment had non-hosts on the conditioned plant species and hosts on the non-conditioned plant species, whereas the congruent environment had hosts on the conditioned plant species and non-hosts on the unconditioned plant species. Parasitoids had to navigate through five non-host infested plants to reach the host-infested plant. Since $C$. glomerata parasitoids do not distinguish between HIPVs induced by host and non-host caterpillars, the conflicting foraging situation caused a prediction error, by guiding parasitoids to non-host infested plants. Especially parasitoids given 3 spaced oviposition experiences, tested in a conflicting situation, spent significantly more time on non-host infested plants and showed a high tendency to oviposit in the non-hosts. As a result, they took significantly more time to find their hosts. Conditioned parasitoids, which were tested in a congruent situation, were more responsive than unconditioned parasitoids, but there was no difference in foraging efficiency between these two groups in the parasitoids that showed a response. We conclude that persistent memories, such as formed after 3 experiences spaced in time, can lead to maladaptive foraging behaviour if the contained information becomes unreliable.
\end{abstract}

Keywords: Cotesia glomerata, learning, foraging efficiency, unreliable information, non-host, oviposition, prediction error, memory 


\section{Introduction}

A high degree of spatial and temporal variation exists in herbivore communities, which makes it challenging for predators to find suitable prey. The way parasitoids use environmental cues to find resources, such as hosts, is of great importance for their realized lifetime reproductive success, a measure of fitness (van Baalen and Hemerik, 2008). An inexperienced female parasitoid is attracted by a range of environmental cues, which have proven their reliability for host finding over generations through natural selection (Stephens, 1993; van Alphen and Bernstein, 2008; Hoedjes et al., 2011). Due to the high degree of both spatial and temporal variation within and between generations in the availability, distribution and abundance of both host and host plant species, these cues can be insufficient to guide parasitoids to their hosts (Stephens, 1993; Vet, 2001). Parasitoids can, however, acquire and process information as they forage, thereby learning how to become more efficient foragers. Parasitoids are known to use a wide variety of olfactory, visual, auditory and tactile cues to obtain and store information on local host presence, distribution and abundance (Vet and Dicke, 1992; Turlings et al., 1993; van Alphen and Bernstein, 2008; Ishii and Shimada, 2009). Acquisition of this information can be achieved through learning, in particular through associative ovipositional learning, where an oviposition in a host becomes associated with various environmental cues, such as herbivore-induced plant volatiles (HIPVs), resulting in associative memory (Bleeker et al., 2006). (Bleeker et al., 2006). Even an encounter with host traces, such as silk and faeces (frass), without the host themselves, results in learning of HIPV's (Geervliet et al., 1998b), albeit that such memories are generally less persistent than after an oviposition experience (Lewis and Martin, 1990; Takasu and Lewis, 2003). With these memories, parasitoids can temporarily adapt their foraging strategy to current local host and host plant availability.

In general, only when multiple learning events occur spaced in time, the learned information is considered reliable enough to adapt foraging behaviour accordingly for a prolonged time. It is then stored in robust long-term memory (LTM), which can last for days (Menzel, 1999; Hoedjes et al., 2011). Moreover, LTM formation is costly in terms of energy expenditure (Menzel, 1999; Mery and Kawecki, 2004), because it depends on protein synthesis (Tully et al., 1994), which is another reason why single learning events usually results in the formation of energetically inexpensive, short lasting memory, naturally decaying within minutes to hours (Menzel, 1999; Hoedjes et al., 2011).

The generalist larval parasitoid Cotesia glomerata, is well known for its ability to learn in both laboratory and (semi-)field studies (Geervliet et al., 1998b; Perfecto 
and Vet, 2003; Smid et al., 2007; De Rijk et al., 2018; Vosteen et al., 2019). Unlike general theory, it consolidates LTM for oviposition events on certain host plants within 4 hours after only a single oviposition in its host Pieris brassicae (Smid et al., 2007). This direct LTM induction is most likely due to the spatial distribution and gregarious nature of this host, since a single encounter with a gregarious host reliably predicts many oviposition opportunities. Indeed, when this parasitoid species encounters a solitary host, $P$. rapae, it does not form LTM, but a less persistent memory type, anaesthesia-resistant memory (Kruidhof et al., 2012). While LTM of a single oviposition wanes over five days, spaced conditioning with 3 ovipositions leads to even more persistent LTM, lasting for more than five days (van Vugt et al., 2015). Thus, experiences with only frass, a single oviposition or three ovipositions spaced in time, each induce different memories with increasing levels of persistence. This memory guides $C$. glomerata to subsequent host patches, but due to the high similarity of HIPV of host and non-host species, these parasitoids are often unable to discriminate between them (Geervliet et al., 1996; Vos et al., 2001; Bukovinszky et al., 2012), even after oviposition experience (Vosteen et al., 2019). The presence of non-host on host plant species has been found to lead to reduced foraging efficiency (Vos et al., 2001; Bukovinszky et al., 2012; de Rijk et al., 2016; Desurmont et al., 2018; Vosteen et al., 2019).

Since environments keep changing, assessment of the reliability of the learned information is a continuous process. Non-hosts might occur on plants previously associated with host presence, where the non-host encounter leads to a predication error; the learned cues do not predict host presence, they have become unreliable. To optimize foraging efficiency, information needs to be processed in an adaptive and integrative way (Hilker and McNeil, 2008), continuously updating memories and acting according to the most reliable information available.

The different levels of memory persistence described above make these parasitoids an ideal model to study the risk of maladaptive foraging behaviour due to persistent unreliable information. Here we conducted a wind tunnel experiment to study how foraging efficiency is affected in the parasitoid C. glomerata, when foraging in an environment, which was either conflicting or congruent with previously learnt information varying in persistence. We confronted the parasitoids with non-hosts on the plant species on which had they previously found their hosts, and hosts on the plant species not encountered before (conflicting foraging situation) or vice versa (congruent foraging situation). We expect that with higher levels of memory persistence, parasitoids will increasingly suffer from reduced foraging efficiency in the conflicting foraging situation, and benefit on the other hand from improved foraging efficiency in the congruent foraging situation. 


\section{Materials \& methods}

\section{Insects}

Pieris brassicae (Lepidoptera: Pieridae) and Mamestra brassicae (Lepidoptra: Noctuidae) caterpillars were reared on Brussels sprouts plants (Brassicae oleracea L. var. gemmifera cultivar Cyrus). Females of the parasitoid wasp Cotesia glomerata (Hymenoptera: Braconidae) were obtained from a yearly re-established culture, and reared on $P$. brassicae caterpillars, to maintain natural foraging behaviour. All insect cultures were maintained at the Laboratory of Entomology, Wageningen University and were reared under the same conditions in a climate-controlled greenhouse with natural light conditions, $21 \pm 1{ }^{\circ} \mathrm{C}$ and $50-70 \%$ humidity. First instar P. brassicae caterpillars were used for parasitoid rearing. Upon emergence of the parasitoid larvae, cocoons were collected and kept in Petri dishes which were put in a climate cabinet $\left(21 \pm 1{ }^{\circ} \mathrm{C}\right.$, L16:D8 photoperiod and $50-70 \%$ humidity). Just prior to emergence the cocoons were transferred to cages $(40 \times 30 \times 30 \mathrm{~cm}$, Bugdorm-1 Insect rearing cage, type DP1000, Megaview Science, Taiwan) with honey and water. Two-day-old females were selected from these cages and kept with honey and water until the start of experiments, when females were 3-5 days old.

\section{Plants}

For experiments three to four weeks old Brassicae nigra L. and Sinapis arvensis $L$. plants were used. Plants were watered daily and were supported by a small green wooden stick and a metal ring to ensure upright growth. Induction of both plant species was accomplished by placing 2 batches of $5 \mathrm{M}$. brassicae $48 \mathrm{~h}$ prior to experiments, or 2 batches of 5 P. brassicae caterpillars $24 \mathrm{~h}$ prior to experiments, on the fourth true leaf of a plant with clip cages. Clip cages were kept upright by attaching each of them to a small green wooden stick ( $30 \mathrm{~cm}$ long, $4 \mathrm{~mm}$ diameter) to prevent the leaf from breaking due to the weight of the clip cage. Besides the clip cages, some cotton wool was wrapped around the base of the leaf to prevent the spread of caterpillars to other leaves once the clip cages were removed. Early first instar $P$. brassicae and late first instar M. brassicae caterpillars were used to infest plants. The difference in age was to obtain similar caterpillar body sizes. $M$. brassicae, however, caused less feeding damage and the induced plants were less attractive to parasitoids after $24 \mathrm{~h}$ induction (personal observation), therefore $M$. brassicae was kept on the plant $24 \mathrm{~h}$ longer than $P$. brassicae to obtain similar damage and attractiveness of plants. After every 3 hours of experiments, plants were replaced. 
Conditioning: learning to associate HIPVs with hosts

Type A :

$20 \mathrm{~s}$ contact with

host frass on

S. arvensis

or

B. nigra

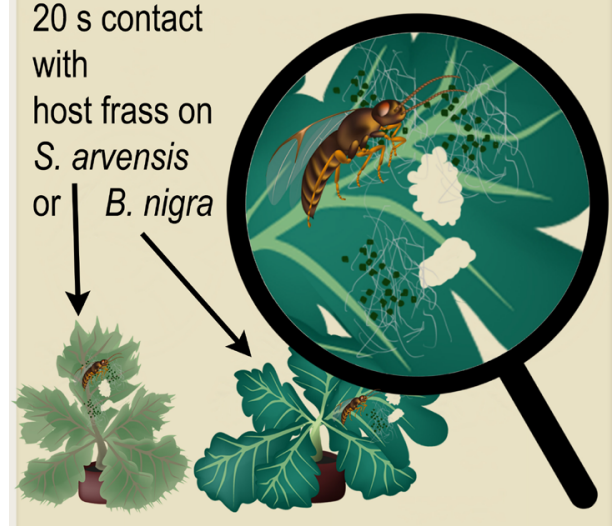

Type B: 1 single host

oviposition, or

C: 3 ovipositions spaced in time

on

S. arvensis

or B. nigra

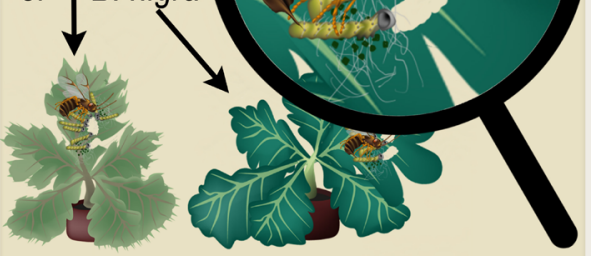

Associative memory 24 hrs after conditioning

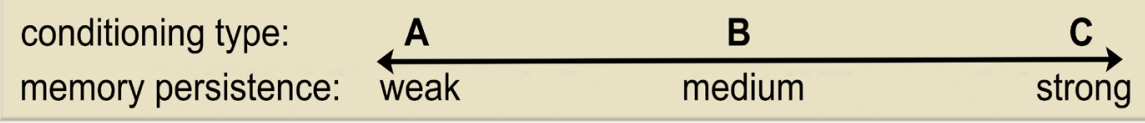

\section{Two test situations for foraging behavior in a wind tunnel:}

Hosts on 1 B. nigra, non-hosts on 5 S. arvensis Hosts on $1 \mathrm{~S}$. arvensis, non-hosts on 5 B. nigra

-neutral for unconditioned wasps

- congruent when conditioned on B. nigra

- conficting when conditioned on S. arvensis

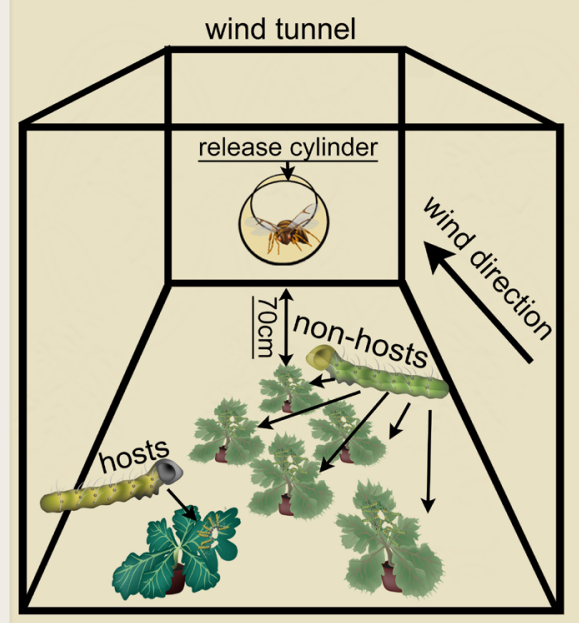

-neutral for unconditioned wasps

- conflicting when conditioned on B. nigra

-congruent when conditioned on $S$. arvensis

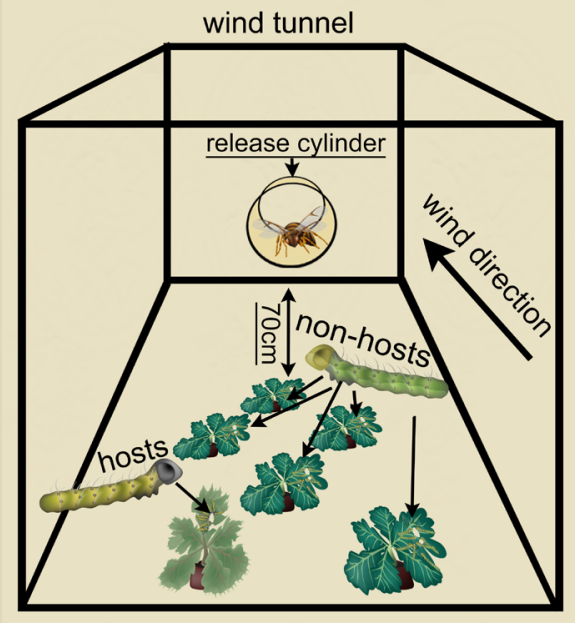


Figure 1. Overview of the conditioning and test procedures. Parasitoids were given a learning experience (top panel), in which they learned to associate herbivore-induced plant volatiles (HIPVs) of Brassicae nigra (dark green plants) or Sinapis arvensis (light green plants) with either a 20 second host frass (Pieris brassicae) exposure, 1 single host oviposition or 3 host ovipositions spaced in time. These conditioning treatments resulted in increasing levels of memory persistence (mid panel, indicated with weak, medium or strong), for either $B$. nigra or $S$. arvensis as predictor for the presence of $P$. brassicae hosts. The next day these conditioned parasitoids were tested in foraging situations created in a wind tunnel (bottom panel), which were either congruent or conflicting with their memory. A conflicting situation consisted of non-hosts (Mamestra brassicae) on five of the plant of the species on which the parasitoids previously experienced hosts or host-frass, and hosts on only one plant of the alternative plant species, located most upwind from the release point. The congruent situation had the same array of 5 plants with non-hosts and one plant with hosts, but in this case the hosts were present on the same plant species on which the previously were conditioned. Unconditioned parasitoids were also tested, for which both foraging situations were considered as neutral. Altogether, this results in 14 different treatments.

\section{Parasitoid conditioning}

A day before conditioning, a $B$. nigra and a $S$. arvensis plant were induced with approximately 200-300 P. brassicae caterpillars spread in batches of approximately 50 caterpillars over the plant leaves. A classical (Pavlovian) conditioning procedure was used, which excludes the host-searching phase, adopted from Bleeker et al. (2006), to give parasitoids an associative learning experience (Fig 1). This procedure consists of giving parasitoids oviposition experience on a plant leaf, where parasitoids learned to associate plant odours as the conditioned stimulus (CS) with suitable hosts as the unconditioned stimulus (US). This type of conditioning is considered a form of classical (Pavlovian) conditioning, where the host-searching phase is excluded.

A total of 7 different conditioning treatments were conducted; parasitoids were kept unconditioned or were given conditioning experience on either the induced B. nigra or $S$. arvensis plant. Conditioning on these leaves consisted of (A) a single leaf damage experience where a parasitoid was transferred from a glass vial to a leaf with host feeding damage. The first instar $P$. brassicae host caterpillars had been removed, but their frass was still present. The parasitoid was allowed to contact the host frass for $20 \mathrm{~s}$, after which it was gently removed with the glass vial. (B) A single oviposition in a first instar $P$. brassicae caterpillar, which was performed as under (A), but now with host caterpillars present. After a single oviposition, the parasitoid was removed with a glass vial. (C) Spaced conditioning consisting of 3 ovipositions spaced in time. It was performed as 3 sequences of single ovipositions, as described for (B), spaced by intervals of 10 minutes, during which the parasitoid remained in the glass vial. Parasitoids were conditioned individually and only ovipositions 


\section{Chapter 3}

lasting longer than 2 seconds were considered successful (Coleman et al., 1999). Fig. 1 shows an overview of these conditioning procedures. While both 1 and 3 ovipositions are expected to induce LTM, spaced conditioning with 3 ovipositions leads to longer lasting, more robust LTM (Smid et al., 2007; van Vugt et al., 2015), with stronger memory persistence (Fig. 1). After conditioning, parasitoids from all treatment groups were placed in small cages $(17.5 \times 17.5 \times 17.5 \mathrm{~cm}$, Bugdorm type 41515, Megaview Science, Taiwan) supplied with water and honey until testing in the wind tunnel the next day.

\section{Wind tunnel set-up}

The experiment was conducted in a wind tunnel as described in Geervliet et al. (1994) with wind speed set to $10 \mathrm{~cm} / \mathrm{s}$, a temperature of $24 \pm 1{ }^{\circ} \mathrm{C}$ and a relative humidity fluctuating between $50 \%$ and $70 \%$. A glass cylinder $(30 \mathrm{~cm}$ long, diameter $15 \mathrm{~cm}$ ) was used as release site and was placed $70 \mathrm{~cm}$ upwind from the first plant. Six plants were placed $15 \mathrm{~cm}$ apart and $10 \mathrm{~cm}$ from the walls of the wind tunnel, five plants infested with the non-host $M$. brassicae and one with host $P$. brassicae, the latter being placed upwind from the five non-host infested plants. Two different foraging situations were created, with either the non-host M. brassicae on B. nigra and the host $P$. brassicae on $S$. arvensis, or vice versa. On a single experimental day both foraging situations were used, each running for 3 hours. Both the order of the foraging situations and the position of the $P$. brassicae plant were alternated daily. The order of the 7 conditioning treatments was randomized, on each experimental day 2 parasitoids were tested per treatment. The 7 conditioning treatments and the 2 foraging situations lead to a total of 14 treatments, each treatment was replicated 15 times. An overview of the conditioning and test procedure of these various treatments is shown in Figure 1.

The wind tunnel was turned on $1-1.5 \mathrm{~h}$ prior to experiments to create stable temperature and humidity values. Just prior to the start of the experiment, plants were positioned in the wind tunnel and clip cages, and their supporting sticks, were removed. Caterpillar movement was restricted to the leaf due to the cotton wool wrapped around the base and caterpillars were counted to make sure 10 live caterpillar would be available. Dead caterpillars were replaced by caterpillars of the same size and age.

Upon the start of the experiment a single parasitoid was capture in a glass test tube $(12 \times 75 \mathrm{~mm})$ and transported from its cage into the glass release cylinder in the wind tunnel. Each parasitoid was given 5 minutes to initiate flight and leave the cylinder. Those that did not fly out of the glass cylinder were taken out of the experiment. 
Parasitoids which directly flew to the ceiling of the wind tunnel were re-released once.

\section{Behavioural observations}

Parasitoid behaviour was recorded on a hand-held computer with The Observer XT 10 software (Noldus Information Technology B.V., Wageningen, The Netherlands) for 15 minutes or until first host oviposition. We used the following behavioural parameters for statistical analysis: foraging time (total time of the behavioural recording), time on non-host patches, number of non-host patch visits and non-host oviposition occurrences. Only behaviour on the actual infested leaves was considered. Furthermore, direct flight (the percentage of parasitoids which only landed on the host plant after flight initiation) and parasitoid response (the percentage of parasitoids initiating flight and orientation to the HIPVs) were also used for statistical analysis.

\section{Statistics}

All statistical analyses were done in $\mathrm{R}$ version 3.4.3 (R Development Core Team 2017). Foraging time was analysed using survival analysis with a cox regression analysis (coxph from the survival package, Therneau and Lumley (2015)), where censored data consisted of parasitoids not finding their host within 900 seconds. Data on time on non-host patches and number of non-host patch visits were analysed using linear mixed models (lme from the nlme package, Pinheiro et al. (2014)) with experimental day as a random factor. Data on the number of non-host patch visits was $\log$ transformed to account for equal variance, time on non-host patches was square root transformed. Presence/absence data on non-host oviposition, direct flight and response was analysed with a Bernoulli glmm (glmer from the lme4 package, Bates et al. (2014)) with day as a random factor.

The statistical models used foraging situation, test plant species and conditioning treatment as fixed factors. Due to an incomplete factorial design, models including unconditioned parasitoids/neutral foraging situation were run without conditioning treatment and vice versa. Differences between groups were analysed with a leastsquare means post hoc comparison with error correction (lsmeans from the lsmeans package, Lenth (2016)). 


\section{Chapter 3}

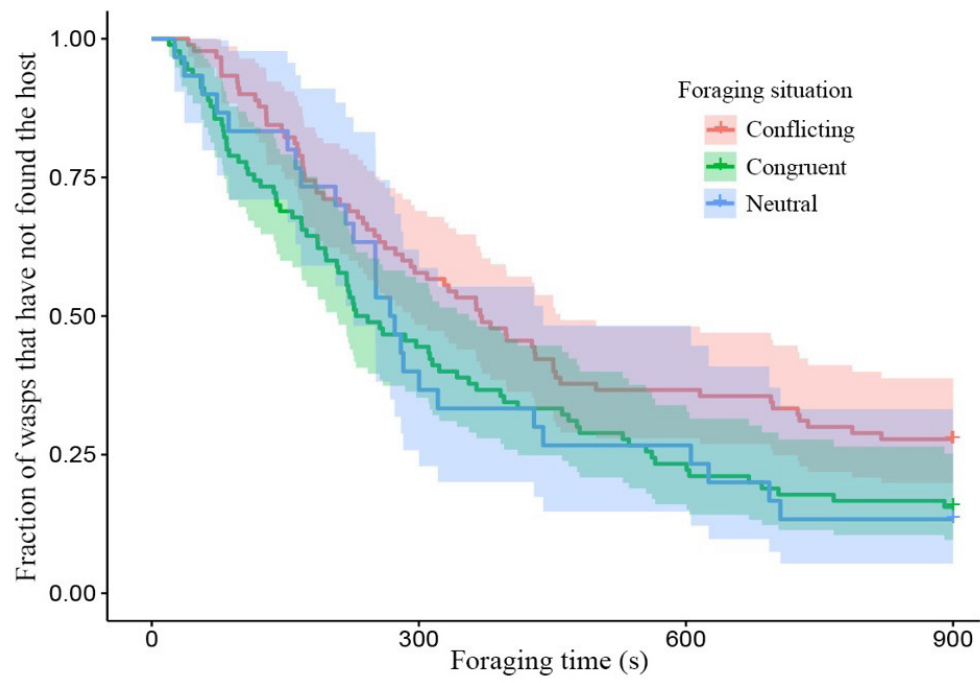

Figure 2. Survival plot of the fraction of parasitoids which have not found the host with foraging time in different foraging situations. Coloured areas around the lines show the $95 \%$ confidence interval (neutral $\mathrm{n}=30$, conflicting and congruent $\mathrm{n}=90$ ). Conflicting and congruent survival curves were significantly different $(z=-2.38, p=0.046)$.

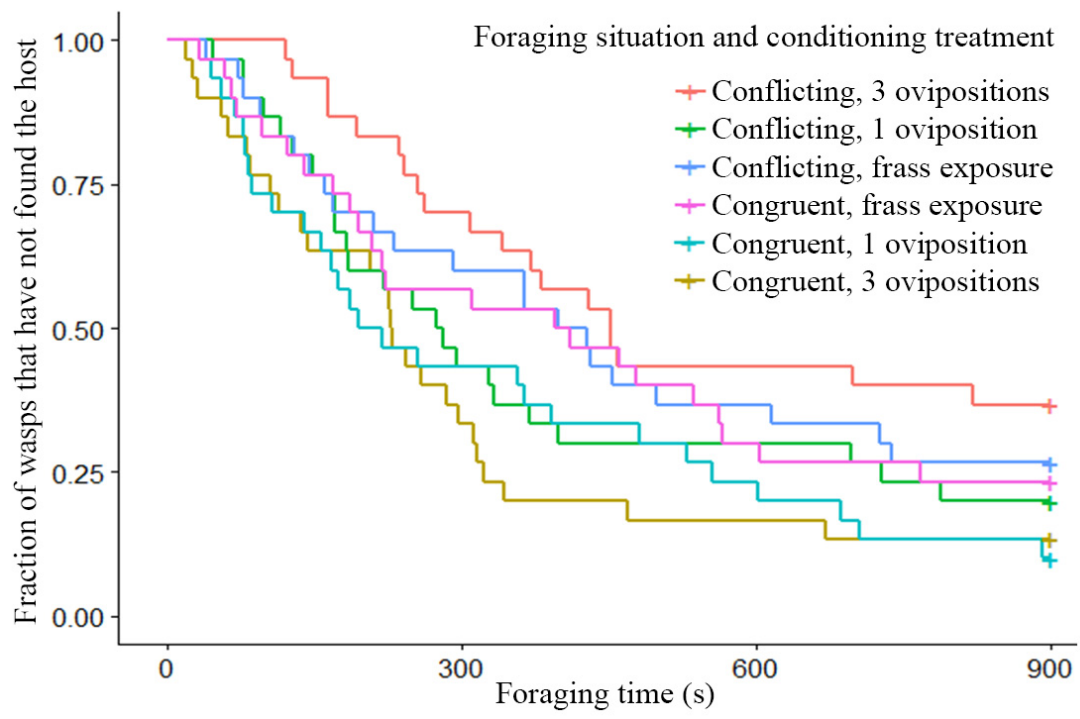

Figure 3. Survival plot of the fraction of parasitoids which have not found the host within the 900 seconds foraging time, given different conditioning treatments and foraging situations $(n=30)$. Parasitoids given 3 spaced ovipositions in combination with conflicting information were significantly different from parasitoids given 3 spaced oviposition in combination with congruent information $(z=-$ $2.96, p=0.036$ ). 


\section{Results}

\section{Effect of foraging situation and conditioning treatment on foraging behaviour}

Parasitoids given conflicting information had more difficulty finding hosts, than parasitoids given congruent information as can be seen by the clear divergence of their survival curves in Figure 2. While conditioning treatment did not have a strong effect on its own, the combination of foraging situation and the conditioning treatment shows clear effects of spaced conditioning with 3 ovipositions (Fig. 3). While 3 ovipositions with congruent information made them the fastest group to find the host, 3 ovipositions with conflicting information resulted in parasitoids being the slowest group to find the host. Since the congruent and conflicting survival curves of frass and a single oviposition show a high degree of overlap, the overall difference between congruent and conflicting foraging situations is mainly explained by the effect of spaced conditioning with 3 ovipositions (Fig. 3).

Assessment of the underlying behavioural components during the foraging period revealed significant differences in the time parasitoids spent on non-host patches. Parasitoids given spaced conditioning with conflicting information stayed more than twice as long on non-host patches, than parasitoids given spaced conditioning with congruent information (Fig. 4). The same pattern was observed for non-host oviposition, where parasitoids given spaced conditioning with conflicting information oviposited 3 times as often in non-hosts, but here the difference between the two spaced conditioning groups had a $p$-value of 0.063 (Fig. 5).

Survival analysis of unconditioned parasitoids, foraging in a neutral situation, switched between the congruent and conflicting conditioned parasitoids within the first 250 seconds (Fig. 2). Thereafter, the unconditioned parasitoids behaved very similar to congruently conditioned parasitoids. Overall, parasitoids foraging in a neutral situation did not behave significantly different from parasitoids foraging in a conflicting $(z=1.76, p=0.183)$ or congruent situation $(z=0.031, p=1.000)$, due to high behavioural variation show in the 95\% confidence interval in Figure 2. Unconditioned parasitoids did make fewer visits to non-host patches, than parasitoids given a conflicting experience $(F=3.04, p=0.049$, Fig. 6a). Furthermore, fewer unconditioned, than conditioned parasitoids responded to HIPVs in the wind tunnel $(z=-5.19, p<0.001$, Fig. 6b). 


\section{Chapter 3}

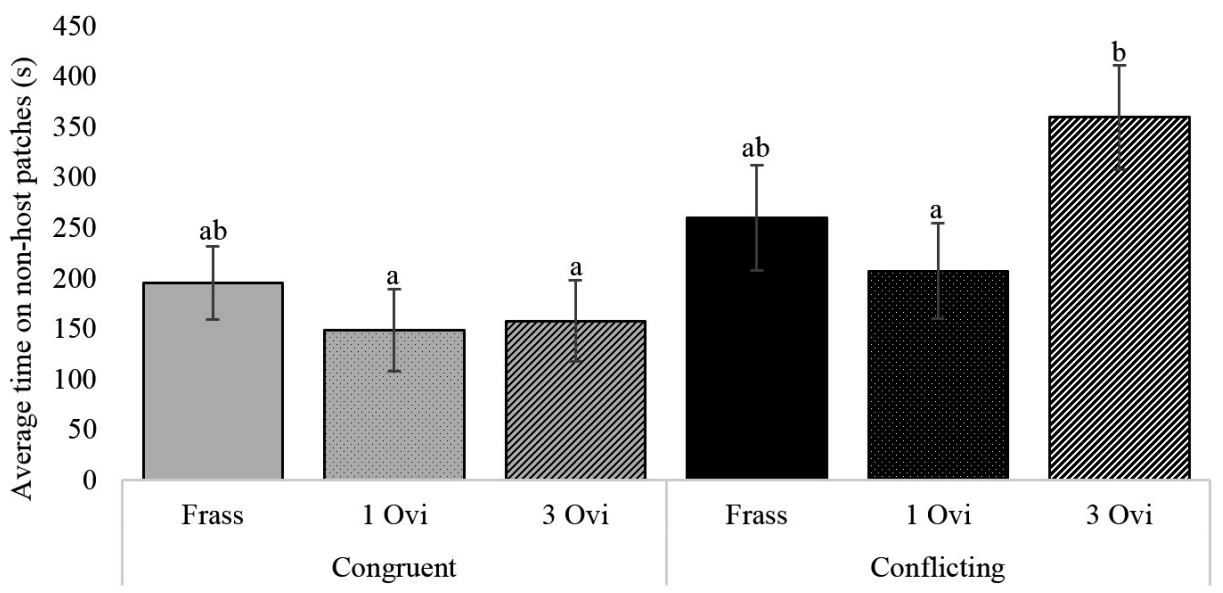

Figure 4. The influence of foraging situation and conditioning treatment on the average time parasitoids spent on non-host patches. Conditioning treatments consisted of a 20 second host frass exposure (Frass), 1 oviposition (1 Ovi) or 3 spaced ovipositions (3 Ovi). Bars with different letters are significantly different $(n=30, \alpha=0.05)$, error bars show the s.e.

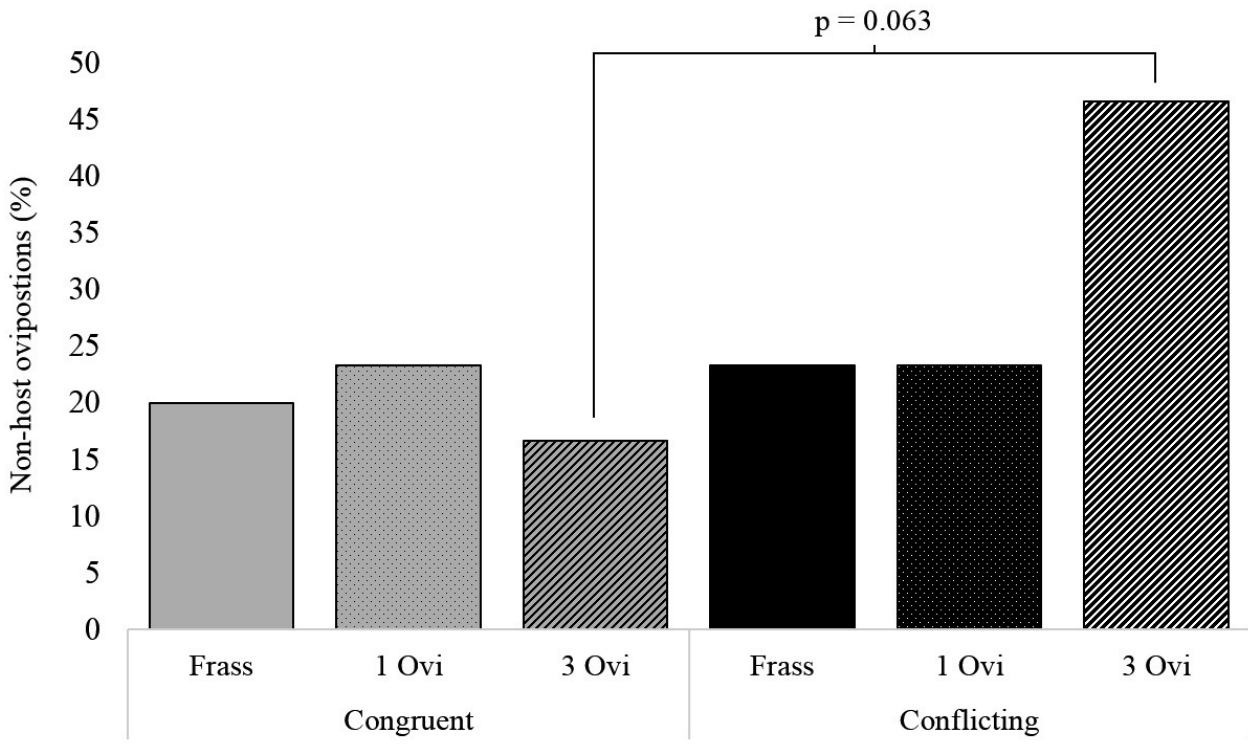

Figure 5. The percentage of parasitoids ovipositing in non-host when given either conflicting or congruent experience with either a 20 second frass experience (Frass), a single oviposition (1 Ovi) or 3 spaced ovipositions (3 Ovi) $(\mathrm{n}=30)$. 
a) 5

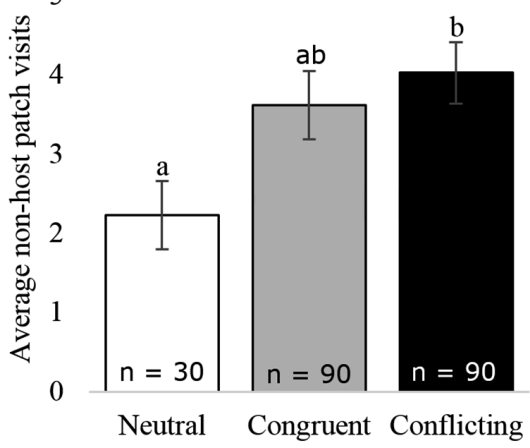

b)

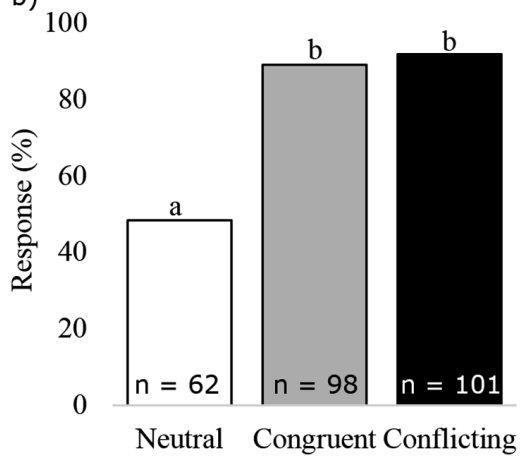

Figure 6. Parasitoid behaviour under different foraging situations: a) average non-host patch visits, b) the percentage of parasitoids responding to the HIPVs in the wind tunnel. Bars with different letters are significantly different $(\alpha=0.05)$, error bars show the s.e.
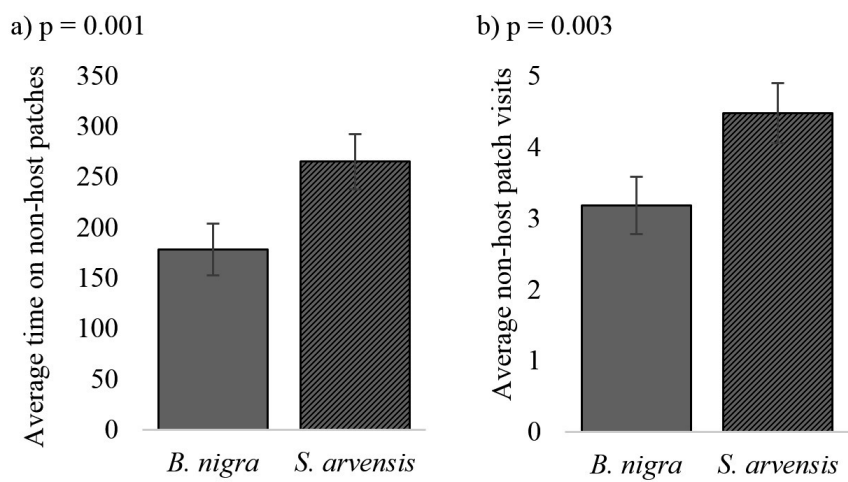

c) $\mathrm{p}=0.002$

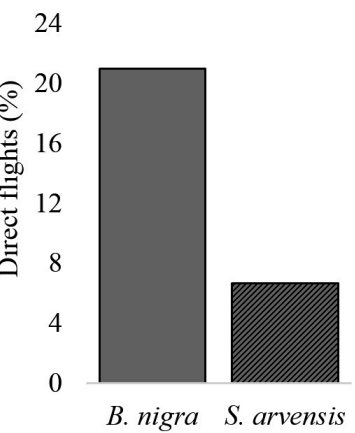

Figure 7. The effect of different host plant species on a) the average amount of time parasitoids spent on non-host patches, $b$ ) the average number of visits to non-host patches and c) the number of direct flights, when the host is found on either Brassica nigra or Sinapis arvensis. Error bars show the s.e. (n $=105)$.

\section{Test plant species effects on foraging behaviour}

The plants species offered during the foraging trail also greatly influenced foraging behaviour. In foraging situations when non-hosts were present on B. nigra and the hosts on $S$. arvensis, parasitoids took longer to find the host $\left(X^{2}=4.87, p=0.027\right)$, they spent more time on non-host leaves $(t=-3.38, p=0.001$, Fig. 7a) and visited non-host leaves more often $(t=-2.61, p=0.010$, Fig. $7 \mathrm{~b})$, than when the host was on $B$. nigra and the non-host on $S$. arvensis. Furthermore, parasitoids performed more 
direct flights when the host was found on B. nigra, than when it was on S. arvensis $(z=2.79, p=0.005$, Fig. $7 \mathrm{c})$.

\section{Discussion}

Natural environments are ever changing, and as a consequence learned information can become outdated and should be forgotten. Since most parasitoids are timelimited, they should utilize their time as efficiently as possible, since the way they exploit their environment directly determines their realized fitness (Baalen and Hemerik 2007). While most studies, focusing on the effect of learning on foraging efficiency, provide the parasitoid with a foraging situation highly similar to what they have been trained in (Geervliet et al., 1998b; Takasu and Lewis, 2003; Bleeker et al., 2006; Smid et al., 2007; Kruidhof et al., 2015; De Rijk et al., 2018; Desurmont et al., 2018), we tested how both reliable and unreliable information affects foraging efficiency in a foraging situation with attractive odour plumes of both hosts and nonhosts. As expected, we found maladaptive foraging behaviour after providing parasitoids with conflicting information, especially when the information has previously proven to be reliable through spaced conditioning. It seems that 3 spaced oviposition experience do not only result in longer lasting memory (Smid et al., 2007; van Vugt et al., 2015), but also results in a stronger focus on the memory content during foraging as the information is considered more reliable. This was reflected in parasitoids taking more time to find hosts and spending more time on non-host patches.

Though learning is generally expected to result in an increase in foraging efficiency, finding more hosts and increasing realized fitness, this most likely only applies if the obtained information is correct. Learning is known to be costly in various ways (Mery and Kawecki, 2004) and our study confirms that persistent unreliable memory involves costs primarily associated with time. However, it is still unclear how the parasitoid will overcome long-term negative effects of this unreliable information. The encounter of a non-host, upon the response to HIPV's previously associated with a host, causes a prediction error and can be considered as a memory extinction event. This event might trigger the formation of additional memory traces, which will diminish the response to the learned cues faster than by natural memory decay (Exton-McGuinness et al., 2015).

Hoedjes et al. (2011) suggested that high cue variability and low cue reliability within a generation should favour the formation of short-lasting memory forms such as STM and ARM rather than LTM. Since LTM is formed after a single oviposition in P. brassicae it seems likely to assume that the HIPVs cue learned in this 
experiment are considered to be of high cue reliability under natural conditions. However, P. brassicae and M. brassicae have overlapping host plant species ranges and share the same habitats. Co-occurrence of these species occurs under natural conditions, on plants in close proximity, but also on the same plant and even the same leaf (Vos et al., 2001). Therefore, it seems likely that non-hosts such as $M$. brassicae are regularly encountered and the cue reliability would be rather low. However, a single encounter with the gregarious $P$. brassicae caterpillars consists of such a high reward value, due to multiple oviposition opportunities, that this might outweigh potential negative effects of cue variability and still facilitates LTM formation after a single oviposition. As mentioned in Koops (2004), if the benefit of correct information is high relative to the cost of the information being unreliable, then the parasitoids should still respond, even if the reliability of the information is relatively low.

The observed foraging behaviour also varied with plant species. When hosts were present on B. nigra and non-host on S. arvensis, parasitoids found the host-infested plants faster, performed more direct flights and spent less time on non-host-infested plants compared with the reciprocal situation. Sinapis arvensis and Brassica nigra are considered sister species (Agerbirk et al., 2008), yet they are apparently different enough to cause substantial differences in foraging behaviour, depending on which plant species contained the hosts or non-hosts. Possibly, B. nigra HIPVs are easier to detect, or are more attractive, than HIPVs of $S$. arvensis, making it easier for parasitoids to find the attractive $B$. nigra host-infested plant among the less attractive HIPVs of $S$. arvensis, than vice versa.

The observation that $C$. glomerata is less efficient at finding hosts in the presence of non-hosts has already been shown in various studies (Bukovinszky et al., 2012; de Rijk et al., 2016), yet so far there has been no mentioning of non-host acceptance under (semi-) natural foraging conditions. Under laboratory conditions, however, Vosteen et al. (2019) and Bukovinszky et al. (2012) found occasional non-host oviposition by C. glomerata in M. brassicae with flight assays. Vosteen et al. (2019) found non-host acceptance levels up to $27 \%$, which seems comparable with our findings. Currently we are investigating to which extent $M$. brassicae is truly a nonhost, if these findings are a side-effect of the test set-up and which circumstances favour non-host acceptance.

In contrast to what we expected, we found that congruently conditioned parasitoids behaved very similar to unconditioned parasitoids. While the study of Kruidhof et al. (2015) showed higher foraging efficiency after associative learning of HIPVs with $C$. glomerata, we did not find this in this study. The main reason why we do not find this positive effect of associative learning is most likely since we discarded 
parasitoids which did not respond within 5 minutes. While response levels of the conditioned parasitoids were around $90 \%$, only $48 \%$ of the unconditioned parasitoids responded within 5 minutes. Oviposition experienced parasitoids are known to be more responsive to HIPVs in general. Giving parasitoids oviposition experience or exposing them to host frass prior to testing is a general way to increase the responsiveness of parasitoid to HIPVs (Geervliet et al., 1998b; Takasu and Lewis, 2003; Bleeker et al., 2006; Peñaflor et al., 2017).

Overall, we conclude that learning unreliable information causes maladapted foraging behaviour, which reduces foraging efficiency under the conflicting test conditions, compared to the congruent test situation. However, parasitoids do not only learn to associate environmental cues with host while foraging, but also with food (Tertuliano et al., 2004; Wäckers et al., 2006). Hungry parasitoid will primarily respond to cues associated with food, while fed parasitoids will primarily respond to cues associated with hosts (Lewis and Takasu, 1990; Luo et al., 2013). Their environment in combination with their physiological state will determine their foraging behaviour and the way they use learned cues. The effect of unreliable memory in relation to food learning and foraging behaviour has not been researched in parasitoids so far, but has been researched in honeybees with colour learning with a food reward. Similar negative effects of persistent unreliable memory were found; 3 learning events led to longer lasting memory than 1 learning event (Menzel, 1968), and bees with 3 learning events returned more often to the previously rewarding colour, which now only supplied tap water, than parasitoids given 1 learning event (Couvillon and Bitterman, 1980).

By learning how parasitoids integrate different kinds of information from their environment to optimize foraging efficiency, we can greatly advance spatial movement models and biological control efforts (van Alphen and Bernstein, 2008; Ishii and Shimada, 2009; Wajnberg et al., 2016). Furthermore, the higher response of parasitoids to local HIPVs after learning is interesting for biological control practices (Prokopy and Lewis, 1993; Giunti et al., 2015; Kruidhof et al., 2019).

\section{Acknowledgements}

This study was supported by NWO open competition grant nr. 824.14.023 to LEMV. 




\title{
Chapter 4
}

\section{Monitoring the effects of information reliability on parasitoid foraging behaviour with a multi-camera system in the field}

\author{
Jessica A.C. de Bruijn ${ }^{1}$ \\ Ilka Vosteen $^{1}$ \\ Louise E.M. Vet ${ }^{1,2}$ \\ Hans M. Smid ${ }^{1}$ \\ Jetske G. de Boer ${ }^{2}$
}

${ }^{1}$ Laboratory of Entomology, Plant Sciences Group, Wageningen University, Wageningen, The Netherlands

${ }^{2}$ Department of Terrestrial Ecology, Netherlands Institute of Ecology (NIOO-KNAW), Wageningen, The Netherlands 


\begin{abstract}
Changing conditions in nature have led to the evolution of behavioural traits that allow animals to use information on local conditions and adjust their behaviour accordingly, for example through learning. Although learning can improve foraging efficiency, the learned information can become unreliable as the environment continues to change. This could lead to potential fitness costs when memories holding such unreliable information persist. We showed previously that persistent unreliable memory, i.e. memory containing unreliable information, reduces the foraging efficiency of the parasitoid Cotesia glomerata under laboratory conditions. Here, we evaluated the effect of such persistent unreliable memory on the foraging behaviour of $C$. glomerata in the field. This is a critical step in studies of foraging theory, since animal behaviour evolved under the complex conditions present in nature. Existing methods for field testing provide little detail on how parasitoids interact with their environment, therefore we developed a novel multi-camera system that allowed us to trace parasitoid foraging behaviour in detail in a semi-field set-up. With this multi-camera system, we studied how persistent unreliable memory affected the foraging behaviour of C. glomerata when these memories led parasitoids to plants infested with non-host caterpillars. Our results demonstrate that persistent unreliable memory not only affected the foraging behaviour of $C$. glomerata under laboratory conditions, but also under field conditions and that different plant species can lead to context-dependent results. Furthermore, persistent unreliable memory made C. glomerata more likely to oviposit in the non-host Mamestra brassica. Overall, we conclude that the multi-camera set-up provided us with new insights into parasitoid foraging behaviour and that persistent unreliable memory can lead to maladaptive foraging behaviour, even under field conditions.
\end{abstract}

Keywords: associative learning, Cotesia glomerata, foraging efficiency, Mamestra brassicae, memory reliability, non-host, plant species 


\section{Introduction}

In nature, environmental conditions are constantly changing. This has led to the evolution of behavioural traits that allow animals to adapt to a dynamic environment (Stephens and Krebs, 1986). By gathering and using information from their environment, animals can adjust their foraging behaviour to local conditions and improve their foraging efficiency (Vet, 2001; Dall et al., 2005; van Baalen and Hemerik, 2008). A high foraging efficiency is expected to improve fitness by increasing lifetime reproductive success, but in most animal species there is no direct link between foraging efficiency and fitness, making it difficult to test this prediction experimentally (van Baalen and Hemerik, 2008). However, in insect parasitoids, which forage for host insects to lay their eggs, foraging behaviour is directly linked to fitness and they are ideal organisms in studies of foraging theory (van Baalen and Hemerik, 2008; Thiel and Hoffmeister, 2009).

Parasitoids lay their eggs in or on other organisms, which function as a host for the developing offspring, ultimately resulting in the death of the host (Godfray, 1994). Due to this intimate relationship, hosts have evolved inconspicuousness and mechanisms to kill deposited parasitoid eggs (Vinson, 1998; van Baalen and Hemerik, 2008). This challenges parasitoids to find hosts that are suitable for offspring development. Due to the direct fitness link between host searching behaviour and offspring production, this behaviour is expected to be under strong selection pressure (Thiel and Hoffmeister, 2009).

Parasitoid host searching behaviour is determined by interactions between external factors, such as host availability and distribution, and internal factors, such as genetics, physiological status and prior experience (Vet et al., 1995; van Baalen and Hemerik, 2008). Learning through prior experience is known to be important in shaping parasitoid foraging behaviour, as it allows the parasitoid to gather, store and use information on local environmental conditions (Vet et al., 1995; Vet, 2001; Hoedjes et al., 2011). When emerging, parasitoid females are genetically adapted to respond to cues that have proven to be reliable over many generations. This information might, however, be insufficient to find hosts due to temporal and spatial variation of host presence in the current environment (Vet, 2001; van Baalen and Hemerik, 2008). Behavioural flexibility, such as the ability to adapt to local conditions through learning, thus provides a benefit in these changing environments. 
Parasitoid can alter their foraging behaviour through learning, by forming an association between environmental cues, i.e. volatile, tactile and visual cues from the host and its habitat (e.g. the host plant), and a host oviposition. During this associative learning process, local information on host identity, density, quality and distribution are stored as memory (Vet, 2001; Hoedjes et al., 2011). Short-term memory is formed directly after an experience, but fades quickly. More persistent, i.e. longer lasting, memory forms, such as long-term memory, can be formed when the value (e.g. host quality) or the encounter frequency of the experience is high (Hoedjes et al., 2011; Smid and Vet, 2016). Both memory forms facilitate temporal adaptation of parasitoid host searching behaviour to local conditions (Smid and Vet, 2016).

Studies on associative learning often focus on a single learning experience, or repetitive experiences, with a single cue and reward (Lewis and Tumlinson, 1988; De Jong and Kaiser, 1991; Bleeker et al., 2006; Smid et al., 2007; Hoedjes et al., 2012; Kawamata et al., 2018) to demonstrate, for example, that a host oviposition on a certain plant species increases the response of parasitoid females to herbivoreinduced volatiles from this plant (Geervliet et al., 1998b). However, in nature, parasitoids gather information from their environment throughout their lifetime. Especially in environments with a high degree of within-lifetime variation, obtained information needs to be continuously re-evaluated to validate its reliability and to maintain a high foraging efficiency. For example, obtained information can become unreliable when plant species that were previously associated with suitable hosts, now contain unsuitable host stages or non-host species. Little is known about how such unreliable memory influences parasitoid foraging behaviour. A recent laboratory study with the parasitoid Cotesia glomerata has shown that persistent unreliable memory, i.e. memory containing information that no longer correctly predicted host presence, caused parasitoids to be highly attracted to non-host infested plants and led to a lower foraging efficiency (de Bruijn et al., 2018b).

Here, we extend this study to the field because a controlled laboratory environment is not representative for natural complex and dynamic foraging conditions, under which learning has evolved (Vet, 2001). Our semi-field study allowed us to evaluate foraging theory in a natural complex environment, with natural background vegetation and insect communities, at a larger spatial and temporal scale. Assessing foraging efficiency is difficult in such experiments and has generally been 
constrained to measuring parasitism levels after a set amount of time (Bukovinszky et al., 2012; Kruidhof et al., 2015; Rijk et al., 2018). Although this shows the final result of the foraging process, it provides no information on how parasitoids spend their time and energy during foraging, how they interact with their environment (Casas et al., 2003), and how experiences shape their behaviour. We therefore developed a novel multi-camera set-up to trace parasitoids during foraging in a complex environment. This set-up allowed us to record detailed foraging behaviour of C. glomerata on host and non-host infested plants. We used this system to test whether persistent unreliable memory, i.e. unreliable information acquired during 3 oviposition experiences spaced in time, leads to decreased foraging efficiency of $C$. glomerata, as observed in the laboratory (de Bruijn et al. 2018b). Because we previously found that plant species had a profound effect on conditioned behaviour, we used a reciprocal approach to test our prediction, with two foraging situations where host and non-host infested plant species were switched.

\section{Materials and methods}

\section{Insects}

Insect cultures originated from individuals collected in Brussel sprouts fields near Wageningen and were refreshed yearly. All insects were cultured at the Laboratory of Entomology, Wageningen University, in a climate-controlled greenhouse at $21 \pm$ $1{ }^{\circ} \mathrm{C}, 50-70 \%$ relative humidity and a L16:D8 photoperiod with both natural and artificial light. Caterpillars of Pieris brassicae (Lepidoptera: Pieridae) and Mamestra brassicae (Lepidoptera: Noctuidae) were reared on Brussels sprouts plants (Brassicae oleracea L. var. gemmifera cultivar Cyrus). Cotesia glomerata (Hymenoptera: Braconidae) was reared on its main host Pieris brassicae. Once $C$. glomerata larvae emerged and cocoons had formed, they were collected in a Petri dish, which was placed in a mesh cage (Bugdorm-1 Insect rearing cage, $30 \times 30 \times 30$ $\mathrm{cm}$, type DP1000, Megaview Science, Taiwan) with water and honey in a climate cabinet $\left(21 \pm 1{ }^{\circ} \mathrm{C}, 50-70 \%\right.$ humidity and L16:D8). For experiments, we used female parasitoids 3 to 5 days after adult emergence. 


\section{Plants}

We used 4-5 weeks old Brassica nigra and Sinapis arvensis plants for experiments. Plants were watered daily and upright growth was facilitated with a wooden stick (30 $\mathrm{cm}$ long, $4 \mathrm{~mm}$ diameter). Host and non-host infested plants were prepared as described in detail in de Bruijn et al. (2018b). Non-host infested plants were infested $48 \mathrm{~h}$ prior to a field trial by placing 5 first instar Mamestra brassicae caterpillars in the bottom of a clip cage and attaching two of these clip cages to the underside of a leaf. Host-infested plants were infested $24 \mathrm{~h}$ before a field trial by placing 2 batches of 5 first instar Pieris brassicae caterpillars on top of a leaf, containing each batch in a clip cage. Fully expanded straight leaves of similar sizes and at similar heights were selected for infestation. Clip cages were supported with a wooden stick. At the start of a field trial, clip cages were removed and cotton wool was wrapped around the stalk of the leaf to prevent caterpillars from dispersing to other leaves.

\section{Field tent set-up}

To observe foraging behaviour of parasitoids, four large mesh tents $(12 \times 12 \times 2.5$ $\mathrm{m})$ were placed in a field near the campus of Wageningen University. The field consisted of natural grass and herb vegetation, with various species of Poaceae, Juncaceae and Cyperaceae and plants such as Bellis perennis, Rumex acetosa and Trifolium pratense. We confirmed that brassicaceous plants were absent. Within each tent, 16 test plants were positioned in an $8 \times 8$ matrix, presented in Figure 1 . On one side of the tent a single host-infested plant was placed, on the opposite side we positioned the parasitoid release plant (always an uninfested B. oleracea plant). All other positions in the matrix were occupied by non-host-infested plants. This layout ensured that parasitoids would encounter several non-host-infested plants after their release, before reaching the host-infested plant. Pots with infested plants were placed on top of the soil within the dense natural vegetation. The height of this vegetation was adjusted to the height of the infested plants, it was kept at approximately $30 \mathrm{~cm}$ through trimming with hedge shears every two weeks.

Since foraging behaviour of parasitoids may be influenced by the plant species used, two reciprocal foraging situations were created. Foraging situation 1 consisted of 14 non-host-infested $S$. arvensis plants, a single host-infested B. nigra plant, and an 
uninfested $B$. oleracea release plant. Foraging situation 2 consisted of 14 non-hostinfested $B$. nigra plants, a single $S$. arvensis host-infested plant and a $B$. oleracea release plant (see Fig. 1). Once plants were placed in the field, clip cages were removed and the number of caterpillars on each plant was checked. Missing caterpillars were replaced to ensure that 10 caterpillars were present on each plant at the start of the experiment. Below each infested plant, a camera was placed that was focussed at the underside of the damaged leaf with caterpillars. Wooden sticks of 15 $\mathrm{cm}$ and $30 \mathrm{~cm}$ were used when necessary to support the horizontal position of the leaf to allow for a good view of the lower leaf surface. Each week, both foraging situations were tested once on different days, with the order of the two foraging situations randomized per week. Plants and cameras were removed from the tent after each trial, and each tent was used only once every 2 weeks. Although we expected parasitoids would die within a week, because no food was provided and very few flowering plants were present, yellow sticky traps (Horiver ${ }^{\circledR}$, Koppert Biological Systems, Berkel en Rodenrijs, the Netherlands) were placed in the tent after each recording to make sure no live parasitoids were present during the next trial.

\section{Colour marking}

To ensure that individual $C$. glomerata females could be recognized during the field trials, parasitoids were given one of 12 colour markings. We used glossy email paints (Revell GMBH, Germany) in the colours white (\#4), yellow (\#12), red (\#31), orange $(\# 30)$, blue (\#50) and green (\#61), which were applied in different 1 to 3 dot patterns to create 12 different colour markings. Due to the fast-drying property of the paint, a thinner (Revell Thinner, Revell GmbH \& Co. KG) was used to maintain a proper consistency of the paint. For colour marking, females were captured individually in a glass vial and briefly placed on ice to anesthetize them, after which an immobilized individual was placed under a microscope and the colour pattern was applied on top of the thorax (see Fig. 1, top panel) with a fine nylon brush. After colour application, the parasitoid was transferred to a small mesh cage (Bugdorm type 41515, $17.5 \mathrm{x}$ $17.5 \times 17.5 \mathrm{~cm}$, Megaview Science, Taiwan) with water and honey and this cage was stored in a climate cabinet until conditioning the following day. 


\section{Conditioning procedure}

Parasitoids were either kept unconditioned or were conditioned with three oviposition experiences spaced by 10 minutes to form persistent memory, as described in de Bruijn et al. (2018b) and depicted in Figure 1. Plants for conditioning were infested $24 \mathrm{~h}$ prior with 200-300 first instar $P$. brassicae caterpillars, distributed in groups of 50 over the leaves of either a $B$. nigra or a $S$. arvensis plant. Colour marked females were allowed to oviposit in host caterpillars on a leaf of either an infested B. nigra or S. arvensis plant, allowing for the formation of an association between oviposition and the plant's volatiles. A female parasitoid was first captured in a glass vial and then transferred to the leaf with hosts. The parasitoid was allowed to oviposit once in a caterpillar, after which it was recaptured in a vial. This procedure was repeated twice at 10-minute intervals. After giving parasitoids three oviposition experiences, they were kept in a small cage with honey and water in a climate cabinet until the start of the field trial the following day.

For parasitoids conditioned on B. nigra, foraging situation 1 (a B. nigra host plant and $S$. arvensis non-host plants) was congruent with the information they had obtained, i.e. they had reliable memory, while for parasitoids conditioned on $S$. arvensis it conflicted with what they had learned, i.e. they had unreliable memory. Foraging situation 2 (a $S$. arvensis host plant and B. nigra non-host plants) conflicted with the information obtained by parasitoids conditioned on $B$. nigra, while for parasitoids conditioned on $S$. arvensis it was congruent. For unconditioned parasitoids both foraging situations were neutral. This reciprocal approach resulted in 6 different treatments, the 3 different experience types (conflicting, congruent and unconditioned), which were tested in each of the 2 foraging situations.

\section{Video set-up}

In order to observe the activity of the parasitoids assigned to these 6 treatments, we used a multi-camera set-up (Cabled 16-dome-system PLUS, Bascom cameras bv, Nieuwegein, The Netherlands), which included 16 dome camera's and a 16-channel hard disk recorder (recorder type PR16K, with a 1 TB hard drive) for data and power transfer (Power over Ethernet, PoE). The cameras (type bsm-pd20) had manual focusing and zoom function $\left(92^{\circ}\right.$ to $\left.28^{\circ}\right)$ and a resolution of $2048 \times 1536$ pixels. A 
UTP cable connected each camera to the hard disk recorder for data transfer and power supply (Figure 1). The video recorder and a 24-inch screen (Phillips 221B) were placed on a table outside the tent. The recorder was connected to the screen via a $16 \mathrm{~m}$ long VGA cable, to allow bringing the monitor inside the tent for manual focusing of each camera during set-up. During each trial, we used 15 cameras that were displayed as live view windows on the screen. A single view window could be used when parasitoid activity was detected by the observer. Since the cameras were directed towards the sky, there was substantial contrast in the recordings, which reduced the saturation of the colour codes on the parasitoids, making it difficult to distinguish these codes reliably. Parasitoid identification was therefore confirmed by an observer inside the tent, upon request of the observer at the screen.

\section{Behavioural observations}

Each experimental day, twelve $C$. glomerata females were transferred from a cage to a glass vial $(28.5 \times 95 \mathrm{~mm}): 4$ conflictingly and 4 congruently conditioned parasitoids and 4 unconditioned parasitoids. This vial was placed directly next to the stem of the release plant. Parasitoids were left to acclimatize for 5 minutes, after which they were released and video recording was started. Parasitoids could forage for a maximum of 5 hours, during which parasitoid presence on host and non-host plants was monitored continuously on the screen. Once a parasitoid was observed to land on a plant (on the monitor outside the tent), its colour marking was checked by the observer in the tent and the time of this first sighting and the associated video channel were noted. Parasitoids had completed the foraging experiment when they found the host caterpillars, after which they were captured and removed from the tent. After 5 hours, the recording was terminated. All trials were done between 7:00 and 17:30, where we planned the 5-hour recording during favourable weather conditions, i.e. $18-28^{\circ} \mathrm{C}$ and no rain. The experiment ran between May and September 2018, with each foraging situation tested 8 times (32 parasitoids per treatment). 


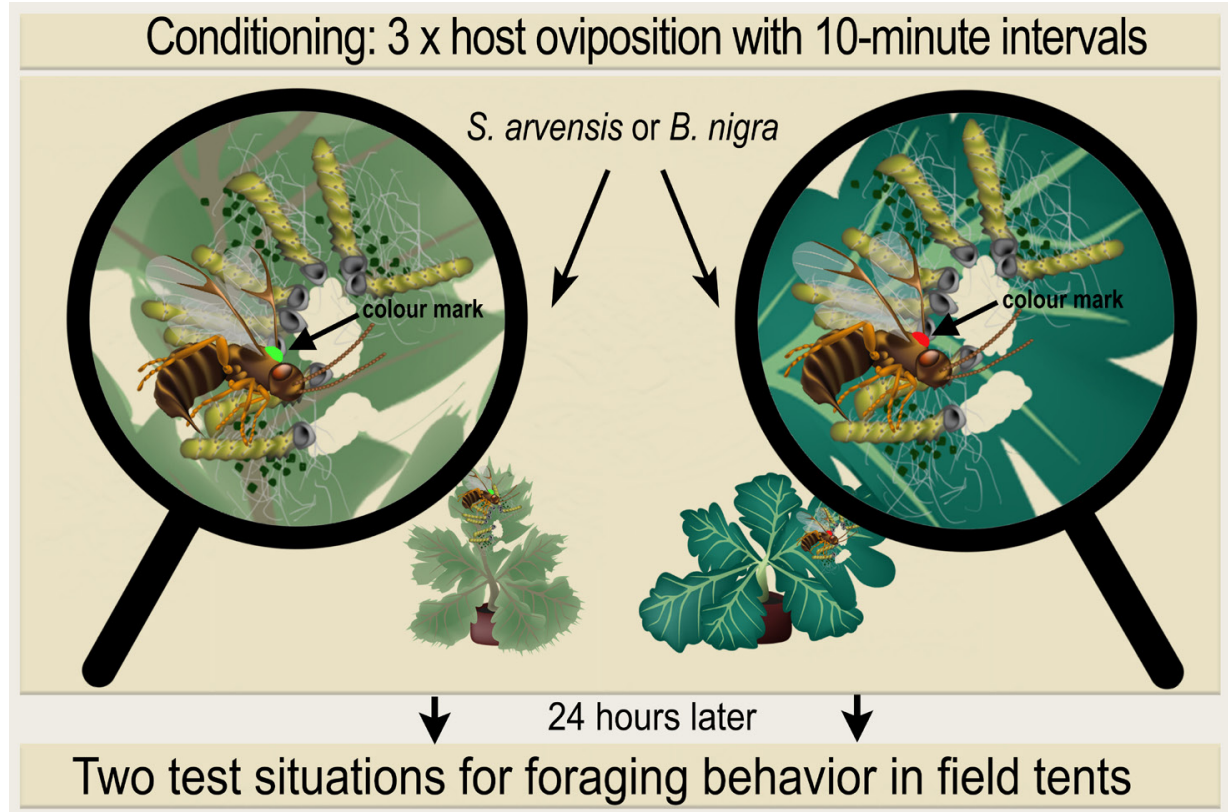

Hosts on $1 \mathrm{~S}$. arvensis, non-hosts on $14 \mathrm{~B}$. nigra Hosts on $1 \mathrm{~B}$. nigra, non-hosts on $14 \mathrm{~S}$. arvensis (shown below in semi-field situation)

-neutral for unconditioned wasps (not shown)

- conflicting when conditioned on B. nigra

-neutral for unconditioned wasps

- congruent when conditioned on S. arvensis

- congruent when conditioned on B. nigra

- conficting when conditioned on S. arvensis

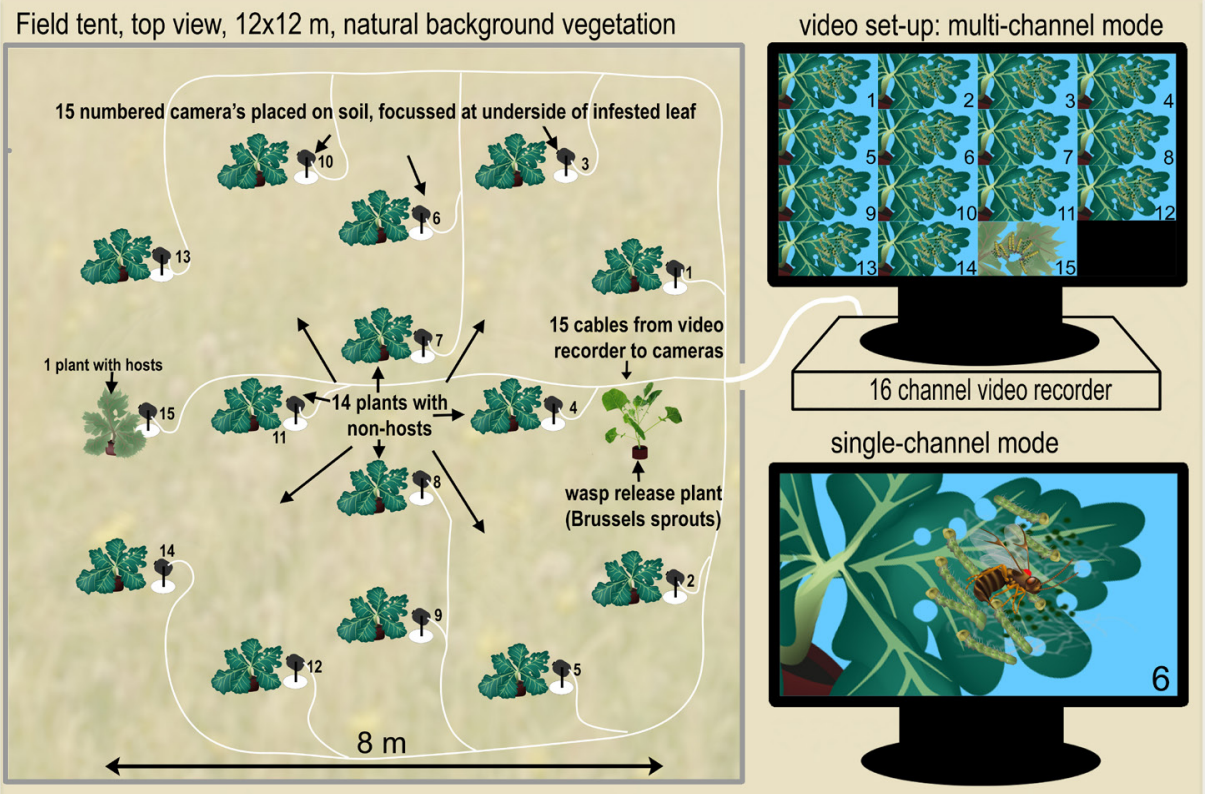


Figure 1. Overview of the experimental approach, including the conditioning method of the colour marked Cotesia glomerata parasitoids (top panel) and the semi field set-up for the observation of parasitoid foraging behaviour with the multi-camera set-up (bottom panels). Colour marked parasitoids were given 3 spaced oviposition experiences on either a Brassica nigra or Sinapis arvensis host-infested plant (top panel) or were kept unconditioned (not shown). They were released in the two test situations $24 \mathrm{hrs}$ after conditioning, as described in the middle panels. The bottom panels show the layout of the field tent, with the locations of the host and non-host infested plants, their associated video cameras, their connection to the video recorder and the display of the 15 video channels, with the multi-channel mode to observe parasitoid activity on all plants and the single-channel mode for a more detailed view. See text for a more detailed description.

\section{Video data collection and processing}

Video files were retrieved from the recorder and stored on a hard disk, after which behavioural data was manually retrieved from these recordings with Windows Media Player (version 12.0.7601.24312, (c) 2009 Microsoft Corporation). Records on the time of first sighting and the associated video channels were used as a starting point to collect data from the video recording on the foraging behaviour of each individual parasitoid. With backtracking, we first determined when the parasitoid landed on the plant for the first time. Since parasitoids would frequently depart from the plant and then land again within a few seconds, often hovering around the infested leaf, backtracking was done until the parasitoid was not seen for 2 minutes. After the first landing, forward tracking was applied to determine all subsequent arrival and departure times to the individual non-host plants and we verified whether, and how often, parasitoids oviposited in the non-host. If the parasitoid was not seen for 2 minutes after the last sighting, it was considered to have left the plant. An arrival and subsequent departure was considered a non-host plant visit when the arrival and subsequent departure times differed by more than 1 second, otherwise it was considered a jump. These jumps were not used in data analyses. Occasionally, more than one parasitoid was observed foraging on a single infested leaf, which led to a confusion of parasitoid identity in a few cases. If there was any doubt about the identity of the parasitoid, its colour code was reported as unknown, and it was not used for further analyses.

These collected data were used, in combination with presence/absence data on whether released parasitoids started to forage and whether they found the host, to determine values of 14 different foraging parameters per individual parasitoid. Each 
foraging parameter is described in Table 1. For the foraging parameter 'time to host' (FP 3), parasitoids were assigned the maximum time of 5 hours when they did not manage to find the host during the recording. These parasitoids may have needed more than 5 hours to find the host, but we analysed this parameter with and without these unsuccessful parasitoids because including them better reflects the difficulty of finding the host.

Table 2. Description of the 14 foraging parameters (FP) and the type of data they consisted of (continuous, count or binary).

\begin{tabular}{|c|c|c|c|}
\hline Code & $\begin{array}{l}\text { Foraging } \\
\text { parameter }\end{array}$ & Type & Description \\
\hline FP 1 & Response & Binary & Whether a released parasitoid was observed foraging \\
\hline FP 2 & Host found & Binary & Whether a foraging parasitoid found the host \\
\hline FP 3 & Time to host & Continuous & $\begin{array}{l}\text { The time from the start of the recording, i.e. time of } \\
\text { release, until the parasitoid landed on the host plant }\end{array}$ \\
\hline FP 4 & $\begin{array}{l}\text { Time until first } \\
\text { landing }\end{array}$ & Continuous & $\begin{array}{l}\text { The time from the start of the recording until the first } \\
\text { landing on an infested plant }\end{array}$ \\
\hline FP 5 & Foraging time & Continuous & $\begin{array}{l}\text { The time the parasitoid was foraging, calculated by } \\
\text { subtracting time until first landing (FP 4) from time } \\
\text { to host (FP 3) }\end{array}$ \\
\hline FP 6 & $\begin{array}{l}\text { NH plant visit } \\
\text { residence time }\end{array}$ & Continuous & $\begin{array}{l}\text { The residence time of a single non-host plant visit, } \\
\text { calculated by subtracting a departure time from the } \\
\text { prior arrival time * }\end{array}$ \\
\hline FP 7 & $\begin{array}{l}\mathrm{NH} \text { plant } \\
\text { residence time }\end{array}$ & Continuous & $\begin{array}{l}\text { The time a parasitoid spent on an individual non-host } \\
\text { plant, where all revisits were stacked * }\end{array}$ \\
\hline FP 8 & $\begin{array}{l}\text { Total NH plant } \\
\text { residence time }\end{array}$ & Continuous & $\begin{array}{l}\text { The sum of all non-host plant visit residence times } \\
\text { of a parasitoid }\end{array}$ \\
\hline FP 9 & Inter-patch time & Continuous & $\begin{array}{l}\text { The time parasitoids used to move from one non- } \\
\text { host plant, i.e. patch, to another, where revisits to the } \\
\text { same plant were ignored * }\end{array}$ \\
\hline FP 10 & Intra-patch time & Continuous & The time it took to revisit the same non-host plant * \\
\hline FP 11 & NH plant visits & Count & $\begin{array}{l}\text { The cumulative number of visits to all non-host } \\
\text { plants }\end{array}$ \\
\hline FP 12 & $\begin{array}{l}\text { NH plants } \\
\text { visited }\end{array}$ & Count & $\begin{array}{l}\text { The number of individual non-host plants that were } \\
\text { visited (out of 14) }\end{array}$ \\
\hline FP 13 & NH oviposition & Binary & $\begin{array}{l}\text { Whether a foraging parasitoid oviposited in a non- } \\
\text { host }\end{array}$ \\
\hline FP 14 & NH ovipositions & Count & $\begin{array}{l}\text { The number of times a parasitoid oviposited in the } \\
\text { non-host }\end{array}$ \\
\hline
\end{tabular}

* Foraging parameters FP 6, FP 7, FP 9 and FP 10 were based on an average time per individual parasitoid. 


\section{Statistics}

$\mathrm{R}$ version 3.5.0 (R Development Core Team 2018) was used for analyses. We used several types of models, which included parasitoid experience as a fixed factor, and when possible, a nested random factor to account for day variation in the different tents (day nested within tent). Separate models were made for the two foraging situations because parasitoid behaviour was clearly context-dependent (see results). Binary data (FP 1,2,13) were analysed using generalized linear mixed-effect models (glmer models, lme4 package, Bates et al. (2014)). Data on unconditioned parasitoids (neutral) were only used in these binary response models because the number of parasitoids that responded was too low to analyse the other parameters (see below). Time to host (FP 3) and time until first landing (FP 4), were analysed using survival analysis with Cox's Proportional Hazard models with frailty (survival package, Therneau \& Lumley,(2015)). Data was censored when parasitoids did not reach the host (FP 3) or when they were not seen landing on an infested plant (FP 4) within 5 hours. Linear mixed-effect models were used to analyse continuous data on foraging time (FP 5, both with and without unsuccessful parasitoids), $\mathrm{NH}$ plant visit residence time (FP 6), NH plant residence time (FP 7), total NH plant residence time (FP 8) and inter- (FP 9) and intra-patch (FP 10) times (nlme package, Pinheiro et al. (2014)). Data was log transformed when residuals were not normally distributed. Count data on NH plant visits (FP 11) was analysed using a glmer model with a Poisson distribution and NH plants visited (FP 12) was analysed with a glmer model with a negative binomial distribution to correct for overdispersion. Data on $\mathrm{NH}$ ovipositions (FP 14) was zero-inflated and therefore analysed with a zero-inflated model with a negative binomial distribution.

\section{Results}

As expected, the two reciprocal foraging situations caused parasitoids to forage in different ways. In foraging situation 1, with the $14 \mathrm{~S}$. arvensis non-host-infested plants and the one B. nigra host-infested plant, only $23 \%$ of the released parasitoids were observed to start foraging on the infested plants (FP 1). In foraging situation 2 , with the $14 \mathrm{~B}$. nigra non-host-infested plants and the one S. arvensis host-infested plant, we observed $42 \%$ of parasitoids foraging. 
Parasitoid response (FP 1), was not significantly influenced by parasitoid experience, although the proportion of unconditioned foraging females (neutral) was lowest in both foraging situations. In foraging situation $1,22 \%$ of parasitoids given conflicting information started to forage, $31 \%$ with congruent information and only $16 \%$ of neutral parasitoids $\left(X^{2}=2.240, p=0.326\right)$. In foraging situation $2,53 \%$ of parasitoids given conflicting information started to forage, $41 \%$ with congruent information and only $32 \%$ of neutral parasitoids $\left(X^{2}=3.203, p=0.202\right)$. Of those parasitoids that started foraging, approximately $50-60 \%$ of parasitoids found the host (FP 2), except in foraging situation 1 , where $80 \%$ ( 4 out of 5 ) of neutral parasitoids found the host. In both foraging situations, the proportion of parasitoids that found the host was not influenced by parasitoid experience (FP 2, Foraging situation 1: $X^{2}=0.109, p=$ 0.947; Foraging situation 2: $X^{2}=1.151, p=0.563$ ).

In foraging situation 1, we observed clear differences in foraging behaviour of parasitoids given conflicting and congruent information (Fig. 2 and 3), although parasitoids given conflicting or congruent information did not differ in how long it took them to find the host on B. nigra (FP 3, Fig. 2A). Parasitoids given congruent information started foraging significantly later (FP 4, Fig. 2C) and their time spent foraging was less than a third of that of parasitoids given conflicting information (FP 5, Fig. 3A). Foraging time was also significantly lower for parasitoids given congruent information, when individuals that did not find the host were excluded (FP $5, F=178.278, p=0.006$ ). Interestingly, parasitoids given conflicting information spent more than 5 times longer on the non-host infested $S$. arvensis plants than parasitoids with congruent information (FP 8, Fig. 3B). They visited twice the number of non-host plants (FP 11, Fig. 3C) and made 3 times more visits to non-host plants (FP 12, Fig. 3D). However, NH plant visit residence time (FP 6, $F=0.135, p=0.724)$ and $\mathrm{NH}$ plant residence time (FP $7, F=2.332, p=0.171) \mathrm{did}$ not differ between parasitoids with conflicting and congruent information. On nonhost infested plants, parasitoids with conflicting information were 5 time more likely to oviposit in the non-host (FP 13, Fig. 3E) and they oviposited significantly more often (FP 14, Fig. 3F) than parasitoids given congruent information. The two groups did not differ with respect to inter-patch time (FP 9, $F=0.079, p=0.790)$ and intrapatch time (FP 10, $F=0.151, p=0.711)$. 


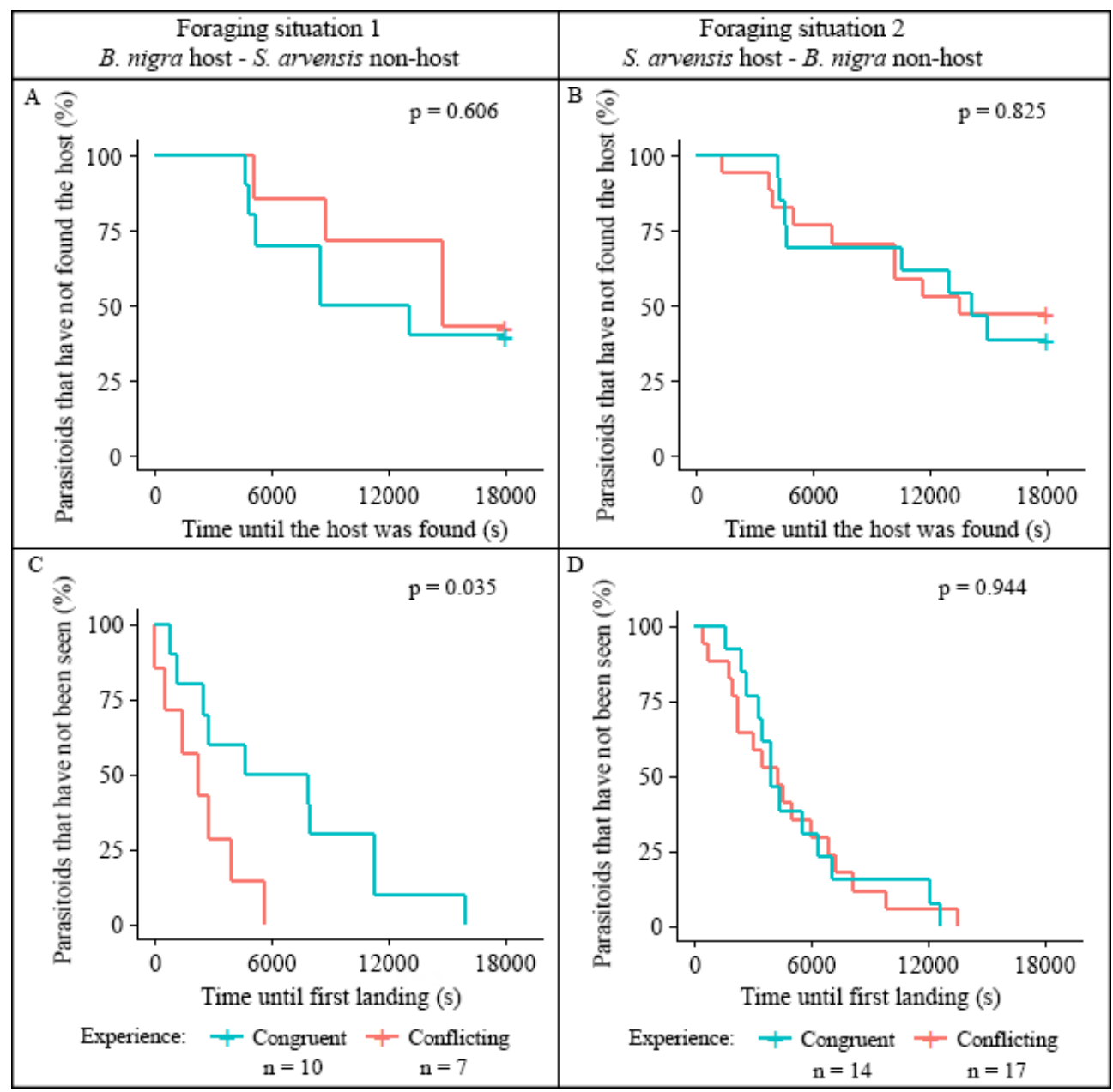

Figure 2. Survival plots of the fraction of C. glomerata parasitoids with congruent and conflicting memory that have not found the host within 5 hours (FP 3) in foraging situation 1 (A) and foraging situation 2 (B), and the fraction of parasitoids that have not been seen, i.e. have not landed on an infested plant (FP 4) in foraging situation 1 (C) and foraging situation 2 (D). These parasitoids foraged in a semi-field set-up with P. brassicae (host) and M. brassicae (non-host) infested plants. P-values are based on Cox's Proportional Hazard models with frailty and show whether the survival curves of parasitoids with conflicting and congruent memory are significantly different. 


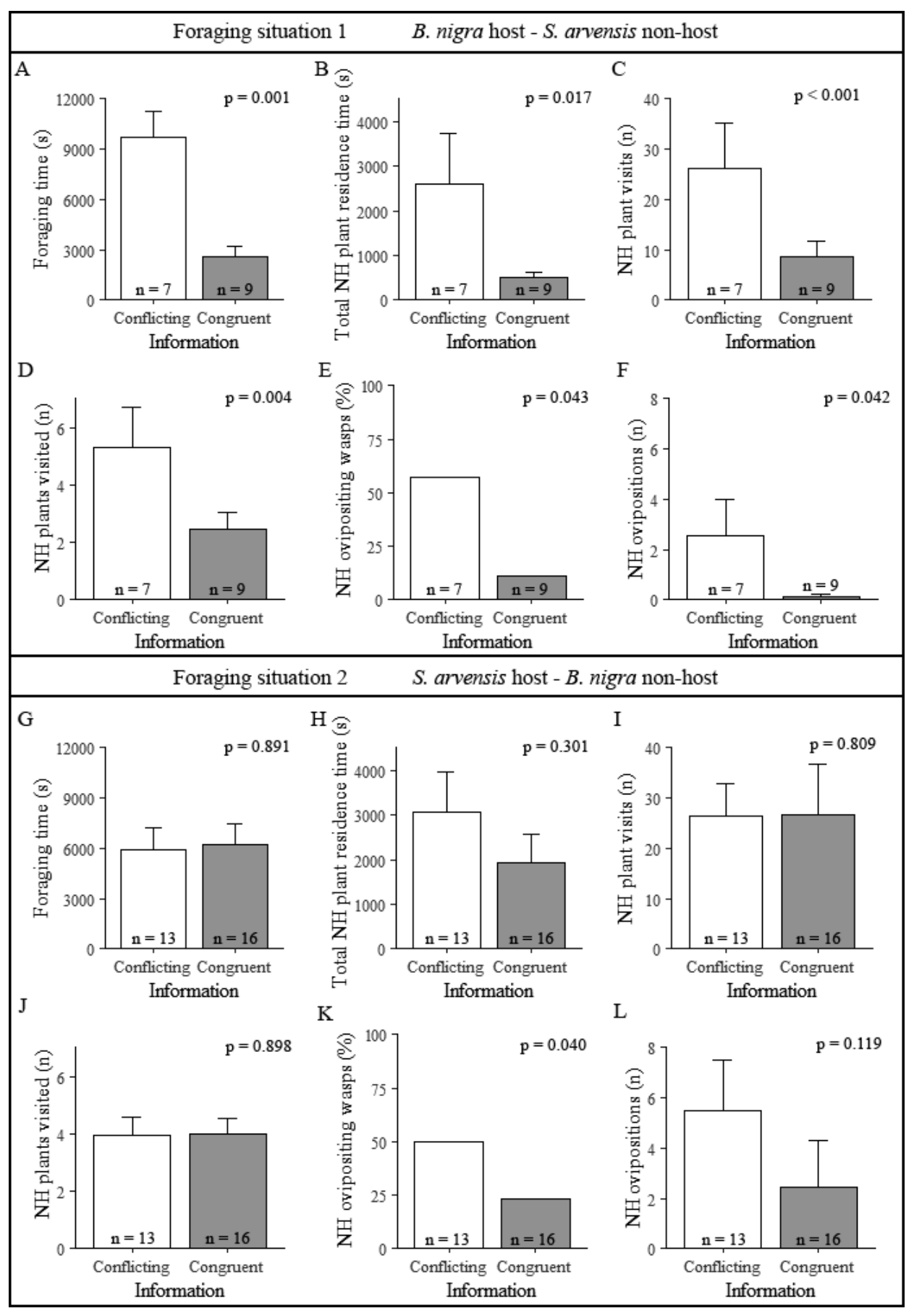


Figure 3. The foraging behaviour of C. glomerata parasitoids with conflicting and congruent memory on and around host- and non-host infested plants. Various behavioural parameters were measured in foraging situation 1 (A-F) and foraging situation 2 (G-L). Panels show how long parasitoids foraged (FP 5, A/G), how much time they spent in total on the non-host (NH) plants (FP 8, B/H), how many visits parasitoids made to NH plants (FP 11, D/J), how many NH plants were visited (FP 12, C/I), the percentage of parasitoids that oviposited in the non-host (FP 13, E/K) and the number of ovipositions in the non-host (FP 14, F/L). Average values are shown with error bars representing the s.e., except for panels $\mathrm{E}$ and $\mathrm{K}$, which show overall percentages. Numbers of parasitoids per treatment are indicated inside bars. P-values show whether the behavioural parameter was significantly different between parasitoids with conflicting and congruent memory.

In foraging situation 2 (Fig. 2 and 3), parasitoids given conflicting and congruent information took equally long to find the host on the infested $S$. arvensis plant (FP 3, Fig. 2B). Both groups started foraging around the same time (FP 4, Fig. 2D) and did not differ in their foraging time (FP 5, Fig. 3G). Parasitoid experience did not influence the number of $\mathrm{NH}$ plant visits (FP 11, Fig. 3J) and number of $\mathrm{NH}$ plants visited (FP 12, Fig. 3I), nor did it influence NH plant visit residence time (FP $6, F=$ 2.204, $p=0.153$ ), NH plant residence time (FP 7, $F=1.268, p=0.273$ ) and total $\mathrm{NH}$ plant residence time (FP 8, Fig. 3H). Furthermore, the two groups also did not differ in inter-patch time (FP 9, $F=0.420, p=0.526$ ) and intra-patch time (FP 10, $F=$ $0.529, p=0.476$ ). Parasitoids given conflicting information were, however, twice as likely to oviposit in the non-host than parasitoids given congruent information (FP 13, Fig. 3K), but the number of ovipositions did not differ (FP 14, Fig. 3L) and was high in both groups.

\section{Discussion}

The multi-camera system provided us with a wealth of information on the foraging behaviour of Cotesia glomerata in relation to information reliability in a complex natural environment. For the first time, we show that persistent unreliable memory can affect foraging behaviour of a parasitoid under field conditions, confirming our laboratory findings (de Bruijn et al., 2018b). Furthermore, we found that the effect of persistent unreliable memory can depend on the foraging situation, i.e. the plant species containing hosts and non-hosts, and that persistent unreliable memory can stimulate non-host oviposition on the conditioned host plant species. In foraging situation 1, where a single Brassica nigra plant was infested with Pieris brassicae 
host caterpillars and 14 Sinapis arvensis plants were infested with Mamestra brassicae non-host caterpillars, parasitoids with persistent unreliable memory (i.e. persistent memory containing unreliable information) spent more time foraging on the non-host plants. These parasitoids encountered a foraging situation that conflicted with their memory, which led to a higher number of non-host plants visited and more non-host plant visits compared to parasitoids with persistent reliable memory, which encountered a congruent foraging situation. Furthermore, 5 times as many parasitoids with persistent unreliable memory oviposited in the non-host, and each parasitoid also oviposited more often in these non-hosts, than parasitoids with persistent reliable memory. Persistent memory, formed by three spaced host oviposition experiences on $S$. arvensis plants, thus proved costly to C. glomerata in terms of time and eggs spent when foraging in an environment where only non-host caterpillars were present on these $S$. arvensis plants. In the reciprocal foraging situation (2), we did not observe time-related effects of persistent unreliable memory, but we did observe that parasitoids with persistent unreliable memory were more prone to oviposit in the non-hosts, as in foraging situation 1. The lack of time-related costs suggests that some aspects of persistent unreliable memory depend on the plant species on which $C$. glomerata forms such memory.

Despite the clear effects of memory reliability on foraging behaviour in our trials, it remains difficult to interpret how foraging efficiency is influenced. When foraging efficiency is defined as the time needed to find a suitable host (from parasitoid release to host found), our results suggest that information reliability does not affect foraging efficiency because parasitoids with conflicting or congruent information did not differ in this respect. However, our multi-camera system revealed clearly that memory reliability had a significant impact on how parasitoids spent their time during foraging. Parasitoids given persistent reliable memory started foraging later on the non-host plants compared to parasitoids given persistent unreliable memory. Even with our advanced multi-camera set-up, we have no information on how these parasitoids spent their time until they were first observed and we do not know whether they were investing time in finding hosts until then. This illustrates the challenge of distinguishing between different parasitoid behaviours, of which host searching behaviour is only one, and makes it difficult to define when such behaviours start and stop, especially under natural conditions. In laboratory studies, initially unresponsive parasitoids are generally excluded when they do not start foraging within 5 minutes (Geervliet et al., 1998b; de Bruijn et al., 2018b), but 
discrimination between actively foraging and initially inactive female parasitoids was not possible in our field set-up. Nevertheless, parasitoids with persistent reliable memory were less active on and around non-host infested plants and were less likely to oviposit in $M$. brassicae than parasitoids with persistent unreliable memory. Furthermore, if time between release and first landing is excluded, clear effects of information reliability on foraging efficiency are observed because parasitoids with persistent unreliable memory spent more time foraging until the host was found.

Plant species, in particular the plant species infested with non-host caterpillars, played an important role when considering the effect of information reliability on parasitoid foraging behaviour, underlining the context-dependency of our findings. In foraging situation 1, it appears that parasitoids with persistent reliable memory, which were conditioned on B. nigra plants, were initially not very attracted to, or even ignored, the non-host infested $S$. arvensis plants because the volatiles of these plants did not match with what they had learned. On the other hand, parasitoids given persistent unreliable memory (conditioned on $S$. arvensis) were directly attracted to the non-host infested $S$. arvensis plants because they had associated host presence with $S$. arvensis volatiles. In foraging situation 2, however, parasitoids of both groups were attracted to the non-host infested B. nigra plants, indicating that the volatiles of these plants were highly attractive to C. glomerata, irrespective of information reliability. We suggest that in this situation the effect of information reliability may be overruled by the high attractiveness, or detectability, of volatiles emitted by B. nigra plants infested with non-host caterpillars. This corroborates previous findings with $C$. glomerata. These parasitoids are known to perceive plant species and cultivars differentially (Geervliet et al., 1996; Poelman et al., 2009), which may be reflected by higher attractiveness or detectability of non-host infested B. nigra and in more time spent or more visits made to these plants in comparison to non-host infested S. arvensis plants (de Bruijn et al., 2018b). It is also possible that the preparedness of parasitoids to learn the volatiles of the two plant species differs (Dunlap and Stephens, 2016). The association formed with B. nigra might have led to a stronger behavioural response to this plant species compared to the association formed with $S$. arvensis. Further experiments with different plant species should reveal whether information reliability is costly in most situations, in terms of foraging time spent on non-host infested plants, or whether our findings are an exception. 
Our experiment, and particularly the use of multiple video cameras, revealed that persistent unreliable memory increased the tendency of $C$. glomerate to oviposit in the non-host $M$. brassicae. Parasitoids given persistent unreliable memory were 2-5 times more likely to oviposit in the non-host in both foraging situations and parasitoids did so significantly more often in foraging situation 1. Although acceptance of this non-host by C. glomerata has been described in several laboratory studies (Bukovinszky et al., 2012; de Bruijn et al., 2018b; Vosteen et al., 2019), this is the first study that clearly shows that associative learning, by means of spaced ovipositional conditioning on a plant, influences the frequency of this seemingly maladaptive behaviour. We were not able to assess the impact of multiple non-host ovipositions on subsequent foraging behaviour and fitness of $C$. glomerata, since our trial ended after five hours. However, based on our findings, we predict that persistent unreliable memory may reduce fitness due to the high number of non-host ovipositions, leading to lower egg loads and energy spent during foraging on plants infested with non-hosts as well as associated risks of predation. Alternatively, subsequent foraging behaviour may be altered by frequent non-host ovipositions, possibly leading to avoidance of these plants. Ultimately, the outcome will depend on plant and caterpillar species composition and distribution in the wider environment, requiring even more complex long-term experiments and more sophisticated tracking of insect behaviour.

Indeed, the application of our multi-camera set-up should be seen as a first step in the development of more advanced methods to track insect movement in the field. The use of cameras, computers and imaging analysis for quantitative studies on insect behaviour has become more popular in recent years and new techniques are being developed rapidly (Reza et al., 2013; Spitzen et al., 2013; Cholé et al., 2015; Zhou et al., 2015; Jiang et al., 2016; de Bruijn et al., 2018a; Gernat et al., 2018; Manoukis and Collier, 2019). The system we used could be further improved by including technologies such as QR code tags and smart cameras that can automatically detect these tags (Gernat et al., 2018), and advanced tracking software that can recognize and track insects despite environmental disturbances, such as wind. Ideally, future technological developments will lead to methods that allow for continuous tracking of insect behaviour in the field, with detailed recordings of their interactions with various organisms in their environment and automated extraction of data from video files. For example, in the present experiment, this could have 
revealed how parasitoids spent their time until they were first observed on a nonhost infested plant.

Overall, we conclude that our approach provided detailed insights in the foraging behaviour of $C$. glomerata in relation to information reliability in a natural foraging situation. We demonstrated that persistent memory, containing unreliable information, can affect the foraging behaviour of $C$. glomerata and that these effects can be context-specific, since volatiles of highly attractive plant species can overrule the effect of information reliability. Furthermore, parasitoid attraction to the nonhost-infested plants and subsequent non-host acceptance behaviour after associative learning is specific to the conditioned host plant species and not a general effect of learning. These findings might have implications for biological control. Many parasitoid species are successfully applied as natural enemies of pest insects, and it has recently been suggested that associative learning prior to parasitoid release may enhance the efficiency of biological control (Giunti et al., 2015; Kruidhof et al., 2019). Future research should focus on testing the effects of information reliability on foraging behaviour of other parasitoid species, further elucidating the influence of plant species, i.e. the context-dependency of our observations, and on further development of more advanced tracking methods, to increase our understanding on how learning shapes insect foraging behaviour.

\section{Acknowledgements}

The NWO open competition grant nr. 824.14.023 awarded to Louise Vet has supported this study. We thank Dr Marjolein Kruidhof for sharing ideas and experience on the use of multiple cameras in a semi field set-up. 



\title{
Chapter 5
}

\section{Memory extinction and spontaneous recovery shaping parasitoid foraging behaviour}

\author{
Jessica A.C. de Bruijn ${ }^{1}$ \\ Louise E.M. Vet ${ }^{1,2}$ \\ Hans M. Smid ${ }^{1}$ \\ Jetske G. de Boer ${ }^{2}$
}

\footnotetext{
${ }^{1}$ Laboratory of Entomology, Plant Sciences Group, Wageningen University, Wageningen, The Netherlands

${ }^{2}$ Department of Terrestrial Ecology, Netherlands Institute of Ecology (NIOO-KNAW), Wageningen, The Netherlands
} 


\begin{abstract}
Insects can alter their foraging behaviour through associative learning, where an encounter with a host or food resource is associated with nearby environmental cues (e.g. plant volatiles), which can improve subsequent foraging efficiency. However, when these associated volatiles are encountered again, the expected food or host resource is not always present. Such an unrewarding experience, termed an extinction experience, can temporarily or permanently change an insect's response to the associated cues. However, little is known about this process in an ecological context and how it shapes foraging behaviour. In this study, we tested whether different types of ecologically relevant extinction experiences would temporarily alter the conditioned host plant preference of the parasitoid wasp Cotesia glomerata, when these parasitoids had learned to associate herbivore-infested plant volatiles with a host oviposition. This was indeed the case when a single extinction experience with host traces was given, while the conditioned behaviour remained unaffected with non-host traces or an oviposition in a non-host in the presence of host traces. Furthermore, by conditioning parasitoids on two different, yet closely related, host plant species, we discovered that the effects of an extinction experience can be host plant species-specific. Our results show that the lack of an oviposition, after contacting host traces, led to the temporary alteration of the conditioned host plant preference in C. glomerata and that effects of extinction experiences can depend on the host plant species used. These results provide novel insights on how different types of extinction experiences, related to hosts and non-hosts, lead to changes in parasitoid foraging behaviour in an ecological context and illustrate the contextdependency of parasitoid foraging behaviour.
\end{abstract}

Keywords: associative learning, Cotesia glomerata, ecology, memory formation, non-host, Mamestra brassicae 


\section{Introduction}

Foraging for resources, such as food and hosts, in a complex environment is a demanding task for insects. Natural environments are highly complex and variable in both time and space, which has led to the evolution of behavioural adaptations to optimize foraging efficiency (Raine et al., 2006; Hilker and McNeil, 2008). One such adaptation is associative learning, where an encounter with a host or food resource is associated with the perception of nearby environmental cues, such as the volatiles and colours of a plant on which the resource is found. These kinds of rewarding experiences can lead to the formation of an associative reward memory, which alters foraging behaviour, and can improve foraging efficiency (Hoedjes et al., 2011). Improved foraging efficiency through associative learning has been shown in various studies, such as learning of landmarks associated with food presence in cockroaches (Durier and Rivault, 2000), food odour learning in fruit flies (Zrelec et al., 2013), floral cue learning in bees (Raine and Chittka, 2008; Russell et al., 2015) and learning of herbivore-induced plant volatiles (HIPVs) to find hosts in parasitoids (Kruidhof et al., 2015). Due to the high complexity and variability of natural environments, associative learning is likely to be common, yet the reliability of the obtained information, i.e. it correctly predicting resource presence, is also likely to be highly variable. Despite the wealth of studies on associative learning, little is known about how variation in information reliability shapes foraging behaviour in insects.

The reliability of an associative reward memory is re-evaluated upon subsequent encounters with the associated cues during foraging. When, after some time, the animal perceives and responds to the associated cues again, and subsequently encounters the expected resources, the learned association will be reinforced, strengthening the reward memory and enhancing the future response to the associated cues (Smid et al., 2007; Lee, 2008). However, insects may also be led to patches containing only resource traces or even unsuitable resources. For example, after associating yellow with nectar presence, bees may be attracted to yellow flowers that no longer contain nectar (Eisenhardt, 2012), or parasitoids may be attracted to non-host infested plants, when non-host induced plant volatiles resemble the volatiles associated with host presence (Bukovinszky et al., 2012; de Bruijn et al., 2018b; Vosteen et al., 2019). When expected resources are not encountered upon 


\section{Chapter 5}

responding to associated cues, the insect gets an extinction experience, i.e. the insect learns that the associated cues do not always reliably predict resource presence.

An extinction experience can change an animal's response to the associated cues (its conditioned behaviour), depending on the strength of the reward memory, and the strength and timing of the extinction experience, as shown in vertebrates (Lee, 2008; Exton-McGuinness et al., 2015) and insects, such as fruit flies (Lagasse et al., 2009) and bees (Eisenhardt, 2012; Eisenhardt et al., 2013). Strong reward memories can be formed by multiple learning experiences spaced in time and/or with resources of high value (e.g. visiting multiple yellow flowers with high quality nectar). These memories tend to be less susceptible to an extinction experience than weaker memories formed with few experiences and/or lower quality rewards (Eisenhardt, 2012; Eisenhardt et al., 2013; Exton-McGuinness et al., 2015).

Insects can form memories that differ in their persistence to account for differences and changes in cue reliability and reward value (Margulies et al., 2005; Hoedjes et al., 2011; Eisenhardt, 2012). This allows for long-term storage of information on highly reliable cues or valuable rewards and short-term storage of cues that have not (yet) proven their reliability or rewards with a low value. As described in Eisenhardt et al. (2006; 2012), memory is first formed as a short-term memory (STM) trace, which disappears within minutes to hours and is relatively sensitive to disturbances such as an extinction experience. STM can then be replaced by more persistent memory traces, such as mid-term memory (MTM) and long-term memory (LTM), which take minutes to hours to consolidate, but last for hours to days and are relatively less sensitive to an extinction experience. The speed of MTM and LTM consolidation can be highly specific to the parasitoid species and the learning experience, which in turn can be host species-specific and potentially plant speciesspecific (Hoedjes et al., 2011; Kruidhof et al., 2012; Hoedjes et al., 2014). These memory dynamics, and the ease with which these associations are formed, are part of a parasitoid's preparedness to learn and is shaped in such a way that it is adaptive in respect to the parasitoid's ecology (Smid and Vet, 2016).

Clearly, the effect of an extinction experience can depend on the timing between the initial conditioning and the extinction experience, because it depends on the sensitivity of the memory trace that is present when the extinction experience is given. Together, the reward memory and the extinction experience either cause no 
behavioural alterations, temporary alterations or permanent alterations (Ecker, 2015; Exton-McGuinness et al., 2015). In case of a strong reward memory or a weak extinction experience, the conditioned behaviour is unlikely to change, while the opposite can permanently alter the conditioned behaviour. Temporary alterations are an intermediate scenario, where the behaviour is altered through the formation of an extinction memory. This extinction memory undergoes the same formation process as the reward memory (as described above), but tends to decay faster, resulting in a spontaneous recovery of the response to the associated cues (Stollhoff et al., 2005).

To date, little is known about these processes in an ecologically relevant context, with foraging insects. Often, synthetic cues and artificial rewards, or punishments, are used to study how extinction experiences shape insect foraging behaviour. For example, in Lagasse et al. (2009), the synthetic volatiles 3-octanol and 4methylcyclohexanol were used in combination with mechanical shock in fruit flies to study the effect of extinction experiences (Lagasse et al., 2009) and synthetic volatiles and/or artificial food rewards have often been used in studies with honeybees and parasitoids (Kaiser et al., 2003; Eisenhardt et al., 2013). It is questionable whether insect foraging behaviour is affected in the same way when natural environmental cues and rewards are used, because synthetic cues and artificial rewards lack natural variation and are not meaningful in an ecological context.

Parasitoid wasps are ideal organisms to study how extinction experiences shape foraging behaviour in an ecological context. They are well known to use environmental cues to forage for their inconspicuous hosts and especially use cues related to the food of their hosts (Vet et al., 1991). The use of herbivore-induced plant volatiles (HIPVs) by parasitoids has been extensively studied, also in respect to associative learning and memory formation (Kaiser et al., 2003; Bleeker et al., 2006; Costa et al., 2010; Hoedjes et al., 2011; Kruidhof et al., 2015; Smid and Vet, 2016; de Bruijn et al., 2018b; Vosteen et al., 2019). Furthermore, parasitoid host searching behaviour is under strong selection pressure, since it is directly related to offspring production and therefore to fitness. This has led to an extensive evolutionary history with various host and host plant-related cues, which has shaped the parasitoid's preparedness to learn (Vet et al., 1990; Smid and Vet, 2016).We expect that it may also affect their response to extinction experiences. Due to the high fitness value of oviposition-related memories, such memories might be more 


\section{Chapter 5}

resistant to extinction experiences than memories related to food or mechanical shock. Extinction experiences are then likely to only lead to temporary alterations of the foraging behaviour, or even no changes at all.

In nature, parasitoids may encounter different types of extinction experiences. Herbivore-induced plant volatiles previously associated with a host oviposition might lead them to plants with non-hosts, or traces of a host or non-host that is no longer present. We expect that an extinction experience with host traces does not lead to changes in behaviour, because host traces are tightly connected with host presence, and contact with host traces can lead to the formation of a reward memory (Hoedjes et al., 2011). Instead of representing an extinction experience, encountering host traces may therefore rather strengthen the reward memory. It may confirm the correctness of the association between HIPVs and hosts, regardless of the absence of the hosts themselves. In contrast, we expect that an extinction experience with non-hosts and their traces will lead to the formation of extinction memory, especially when an associative reward memory is still in the sensitive STM phase. Encountering non-host traces clearly indicates that the learned information does not reliably predict host presence, thus representing a stronger extinction experience. To test these hypotheses, we used Cotesia glomerata, a larval endoparasitoid of caterpillars of the large cabbage white Pieris brassicae (Laing and Levin, 1982). Cotesia glomerata has an innate response to HIPVs of certain host plants, but its preference can be altered through associative learning of HIPVs of other plant species (Geervliet et al., 1996; Geervliet et al., 1998b), which can result in the formation of LTM (Smid et al., 2007). Here, we exposed C. glomerata to different types of extinction experiences 10 minutes after conditioning, when an STM memory trace is present but LTM is not consolidated yet (van den Berg et al., 2011), to test for the disappearance and spontaneous recovery of the conditioned host plant preference. Furthermore, we tested whether these extinction experiences can lead to plant species-specific results, by conditioning parasitoids on two different, yet closely related, host plant species. 


\section{Materials and methods}

\section{Insects}

All insect colonies were reared at the Laboratory of Entomology, Wageningen University. Insects were kept in a greenhouse with a photoperiod of L16:D8 (both natural and artificial light), at $21 \pm 1{ }^{\circ} \mathrm{C}$ and $50-70 \%$ relative humidity. Colonies were re-established each year with individuals collected from cabbage fields around Wageningen. Cotesia glomerata (Hymenoptera: Braconidae) was reared on first instar caterpillars of Pieris brassicae (Lepidoptera: Pieridae). Both P. brassicae and Mamestra brassicae caterpillars were reared on Brussels sprouts plants (Brassica oleracea $\mathrm{L}$. var. gemmifera cultivar Cyrus).

Newly formed C. glomerata cocoons were collected from the rearing and transferred to mesh cages $(30 \times 30 \times 30 \mathrm{~cm}$, Bugdorm-1 Insect rearing cage, type DP1000, Megaview Science, Taiwan) with water and honey, which were placed in a climate chamber set at $21 \pm 1{ }^{\circ} \mathrm{C}, 50-70 \%$ relative humidity and a photoperiod of L16:D8. Two-day-old females were collected from these cages and transferred to a clean cage with water and honey. Females were used in experiments when they were 3-5 days old.

\section{Plant induction}

We used black mustard (Brassica nigra) and red cabbage (Brassica oleracea var. rubra (DC) cv. Langedijker bewaar) plants in experiments when they were four weeks old. To induce emission of herbivore-induced plant volatiles, plants were infested 24 hours before use with either $1^{\text {st }}$ instar host caterpillars (P. brassicae) or $1^{\text {st }}$ instar non-host caterpillars (M. brassicae). Plants used to test host plant preference were infested by placing a group of 20 host caterpillars on the third leaf of a black mustard or red cabbage plant. Plants used for conditioning were infested with a group of approximately 50 host caterpillars on each of the four youngest fully expanded leaves. Black mustard and red cabbage leaves used during the extinction phase were infested with either a group of 30 host caterpillars or 30 non-host caterpillars, placed on the two youngest fully expanded leaves of a plant. The non-host caterpillars were 


\section{Chapter 5}

placed in two clip cages, each with 15 non-host caterpillars, which were attached to a single leaf.

\section{Wind tunnel assay}

Parasitoids were tested in a wind tunnel as described by Geervliet et al. (1994) and depicted in Figure 1c, at $24 \pm 1{ }^{\circ} \mathrm{C}$ with a relative humidity between $50-70 \%$ and a wind speed of $10 \mathrm{~cm} / \mathrm{s}$. Two test plants, a host-induced black mustard and hostinduced red cabbage plant, were placed $70 \mathrm{~cm}$ upwind from the release point, $30 \mathrm{~cm}$ from each other, and $10 \mathrm{~cm}$ from the walls of the wind tunnel.

Upon testing, a parasitoid was released in the centre of the glass release cylinder (30 $\mathrm{cm}$ long, $15 \mathrm{~cm}$ diameter). The parasitoid was given 5 minutes to depart from the release cylinder and land on an induced plant. Once landed its choice was recorded and it was removed from the wind tunnel. Parasitoids that directly flew to the wind tunnel ceiling, upon departure from the release cylinder, were recaptured and released once more. Parasitoids that did not land on an induced plant within 5 minutes were removed from the experiment. Test plants were used for a maximum of 4 hours, except in the spontaneous recovery experiment (see below), where we used a new set of plants for each time point, to minimize effects of decreased plant volatile release over time.

\section{Parasitoid conditioning}

\section{Unconditioned and conditioned host plant preferences}

We first assessed preference of $C$. glomerata for black mustard and red cabbage plants by testing unconditioned parasitoids, after which we tested conditioned parasitoids. Conditioning was done as described in Bleeker et al. (2006) and depicted in Figure 1a, by releasing a single unconditioned parasitoid on an infested leaf of either a black mustard or red cabbage plant, and allowing it to oviposit in a $1^{\text {st }}$ instar host caterpillar once. After the oviposition both the parasitoid and the parasitized caterpillar were removed from the leaf. The parasitoid was then contained in a glass vial for 10 minutes, after which it was tested in the wind tunnel (Fig. 1c). Each day, 8-10 parasitoids were tested, either unconditioned, or black mustard and red cabbage 
conditioned parasitoids. Since unconditioned behaviour is known to be more variable than conditioned behaviour, we performed more replicates with this group to get an accurate estimate of the host plant preference.

\section{Extinction}

For the extinction experiment, parasitoids were individually conditioned with a rewarding experience on either black mustard or red cabbage as described above (Fig. 1a) and then placed in groups of four in a mesh cage $(17.5 \times 17.5 \times 17.5 \mathrm{~cm}$, Bugdorm-41515 Insect Cage, type BD41515, Megaview Science, Taiwan) with water and honey for 10 minutes. They were then each given a different extinction experience on their conditioned plant in a Petri dish $(94 \times 16 \mathrm{~mm}$, Greiner bio-one, Germany) as depicted in Figure 1b. The four extinction treatments included: 1) host traces (HT), where the parasitoid was exposed to host traces (frass and silk) on the conditioned plant species for 15 minutes; 2) non-host traces (NHT), where the parasitoid was exposed to non-host traces on their conditioned plant for 15 minutes; 3) non-host oviposition (NHO), which consisted of two steps, first the parasitoid was given a single oviposition in a non-host caterpillar on a leaf with host traces. After that, the parasitoid was placed in a clean Petri dish for 15 minutes; 4) a control (C), where the parasitoid was directly placed in a clean Petri dish for 15 minutes.

Both the conditioning and the extinction were given on a leaf in a closed Petri dish to limit emanation of HIPVs into the laboratory and to keep the leaves fresh for a longer period. Leaves were detached from the plant just prior to their use. In the conditioning phase, one leaf was used for each group of 4 parasitoids, after which it was discarded. In the extinction phase, two leaves with host traces (treatments 1 and 3) and one with non-host traces (treatment 2) were used. Petri dishes were kept closed at all times, except for a short moment when the parasitoid was introduced. We placed a small (approximately $0.8 \mathrm{~cm}$ ) wet cotton ball in clean Petri dishes in treatments 3 and 4 to reduce the static properties of the Petri dish and elevate relative humidity, to make conditions more comparable to Petri dishes with a leaf. After the extinction experience, parasitoids were transferred to cages with water and honey for 10 minutes before testing. Four parasitoids of each of the 8 treatments (4 treatments per plant species) were tested in the wind tunnel per day. 
a) Conditioning on host-infested leaf in Petri dish

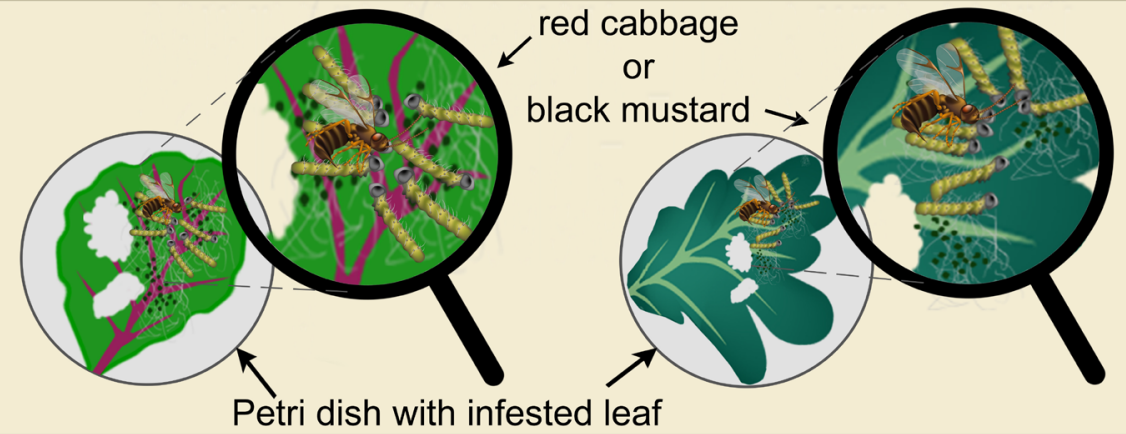

b)
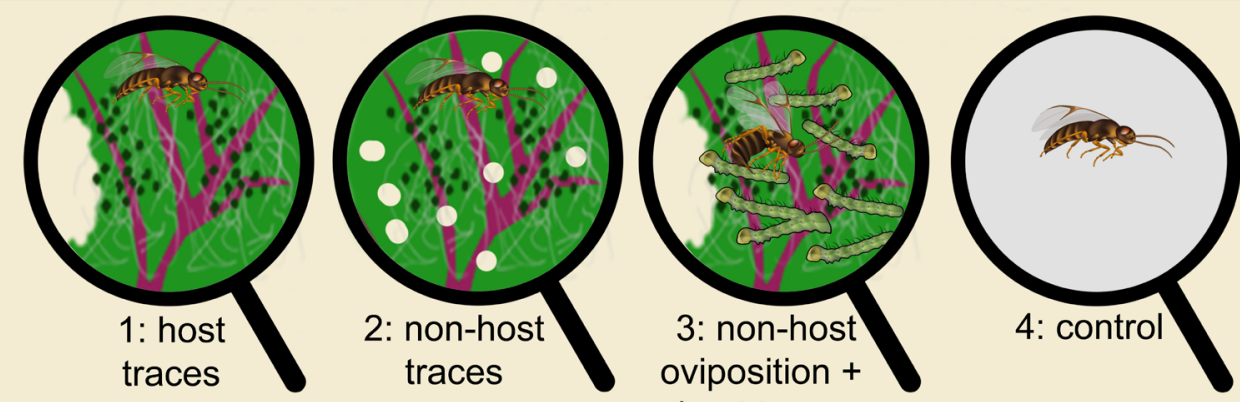

host traces

c)

Memory retention testing in a wind tunnel

Memory formation: test memory retention 10 minutes after conditioning

Memory extinction: test memory retention 10 minutes after extinction type 1, 2, 3 and 4

Spontaneous recovery: test memory retention

10 minutes, 1 hour and 4 hours after extinction type 1 and 4

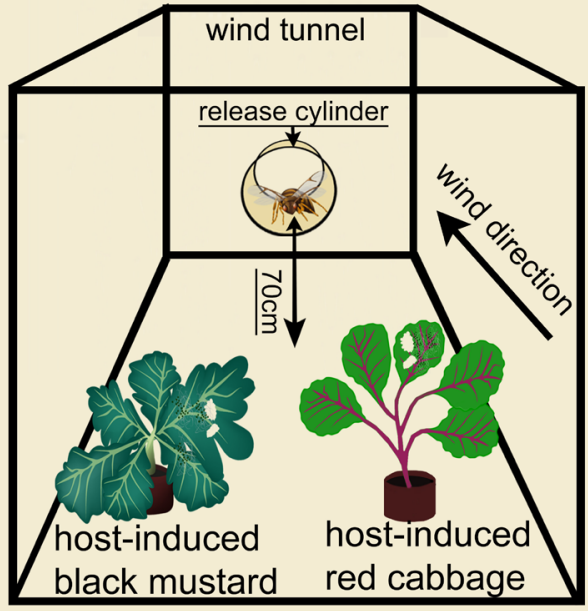


Figure 1. Schematic representation of the experimental approach. The enlarged view, represented by a looking glass, shows all components of the conditioning: the presence of the parasitoid, P. brassicae host caterpillars (yellow with black head), M. brassicae non-host caterpillars (green with yellow head), silk and frass as host traces (white strands and green dots) and feeding damage (gaps in the leaf surface). a) Conditioning of the parasitoids on a host-infested leaf of either red cabbage or black mustard in a Petri dish. b) The 4 different extinction treatments, given in a Petri dish on a leaf of their conditioned plant 10 mins after conditioning: (1) experiencing host traces; (2) experiencing non-host traces (note the difference in feeding pattern of the solitary-feeding non-hosts $M$. brassicae with the gregariouslyfeeding hosts $P$. brassicae caterpillars); (3) oviposition in a non-host placed on a leaf with host traces; (4) control treatment in a clean Petri dish. c) Testing memory retention in a wind tunnel. The wind tunnel (right) is shown in a frontal view, with the host plants with host feeding damage placed upwind, and the release cylinder with a parasitoid placed $70 \mathrm{~cm}$ downwind from the plants. Each parasitoid was tested only once. The same set-up was used for measuring initial memory formation, the effect of the four different memory extinction treatments and spontaneous recovery.

\section{Spontaneous recovery}

For the spontaneous recovery experiment, groups of 12 parasitoids were individually given a rewarding experience on black mustard in a Petri dish, as described before (Fig 1a). They were then kept in a cage for 10 minutes with water and honey, after which 6 parasitoids were given an extinction experience with host traces on a black mustard leaf in a Petri dish for 15 minutes, while the other 6 parasitoids were assigned to the control treatment and given no extinction experience, as described above in extinction treatments 1 and 4 (Fig. 1b). After 15 minutes, parasitoids were transferred to clean cages with water and honey. We determined conditioned host plant preference in a wind tunnel after 10 minutes, 1 hour or 4 hours, by randomly assigning two parasitoids of each treatment to these three test times (Fig. 1c). This procedure was repeated 3 times, resulting in 6 parasitoids per treatment per day.

\section{Statistical analysis}

All statistical analyses were done in R 3.5.0. The binomial data on host plant choice was transformed to continuous data by expressing the binomial data of each experimental day as the percentage of choice for the conditioned host plant, or black mustard in case of unconditioned parasitoids, to form day values. For example, if $70 \%$ of parasitoids chose the conditioned host plant on an experimental day, then the value 70 represented a single replicate. Host plant preferences (within treatments), 


\section{Chapter 5}

were tested with a one-sample t-test in case of normally distributed data and otherwise with a one-sample Wilcoxon signed rank test. In both cases the distribution was compared to a no-preference situation $(\mu=50)$. Due to a lack of variation (identical day values) after conditioning on red cabbage (Fig. 2), 0.000001 was added to one of the day values to allow statistical analysis. Differences between treatments in the experiments on conditioned preferences, extinction and spontaneous recovery, were compared with one-way ANOVAs, after checking for normality and equal variance. Post hoc pairwise comparisons were done with a Tukey's test (lsmeans package, Lenth (2016)), or a Dunnett's test (DescTools package, Signorell (2016)). A Tukey's test was used in the spontaneous recovery experiment to check for memory decay over time in the control group. Dunnett's tests were used to compare treatment groups to controls.

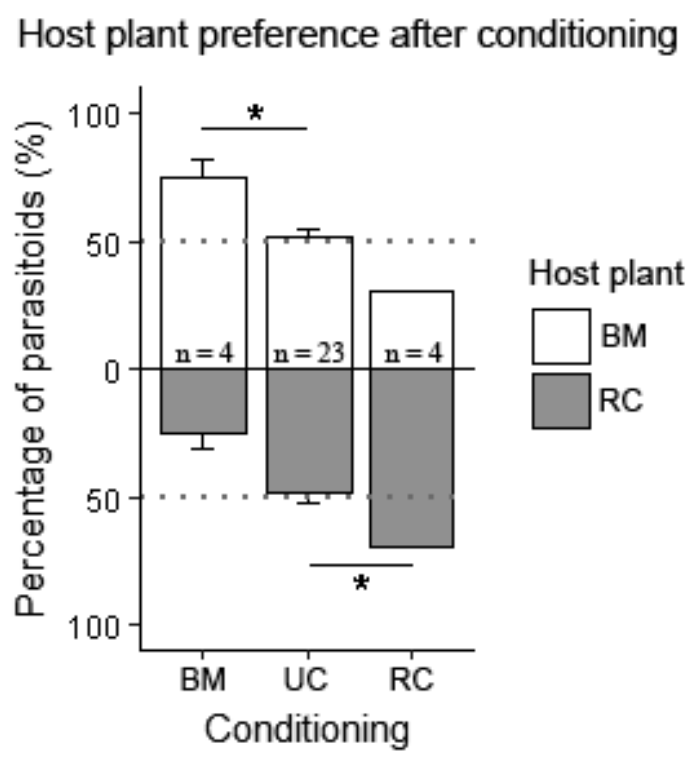

Figure 2. Host plant preference of unconditioned (UC), black mustard (BM) conditioned and red cabbage $(\mathrm{RC})$ conditioned parasitoids. A significant difference $(p<0.05)$ between conditioned and unconditioned parasitoids is indicated with a line and an asterisk above/below the compared bars. 


\section{Results}

\section{Unconditioned and conditioned host plant preferences}

Unconditioned $C$. glomerata females had no preference for either black mustard or red cabbage plants infested with host caterpillars $(t=-0.295, p=0.770)$. Both conditioned groups, showed a significant shift in preference towards the conditioned plants compared to the unconditioned parasitoids (Fig. 2, black mustard: $p=0.012$, red cabbage: $p=0.045$ ).

\section{The effect of different types of extinction experiences}

We determined whether a conditioned preference for one plant species was influenced by an extinction experience 10 minutes thereafter. Parasitoids conditioned on black mustard lost their host plant preference after an extinction experience with host traces $(V=22.5, p=643)$, and the behaviour of these parasitoids was significantly different from the control group (Fig. $3 \mathrm{a}, p=0.032$ ). The host plant preference was maintained when parasitoid were given an extinction experience with non-host traces $(V=28, p=0.019)$ or a non-host oviposition $(t=2.756, p=0.020)$ and their behaviour was comparable to the control group (Fig. 3a, NHO: $p=0.949$, NHT: $p=0.986)$.

In case of conditioning with red cabbage, none of the of extinction groups was significantly different from the control group (Fig. 3b, NHT: $p=0.346$, NHO: $p=$ 0.749). The preference for the conditioned host plant was maintained after an extinction experience with host traces $(t=3.429, p=0.006)$, but it was lost after an extinction experience with non-host traces $(t=0.510, p=0.621)$ or a non-host oviposition $(t=1.297, p=0.224)$.

\section{Spontaneous recovery}

Parasitoids conditioned on black mustard maintained their preference for this plant species up to 4 hours after conditioning (Fig. 4) and this preference did not wane in this period $(F=0.723, p=0.496)$. 
a) Host plant preference after extinction with black mustard

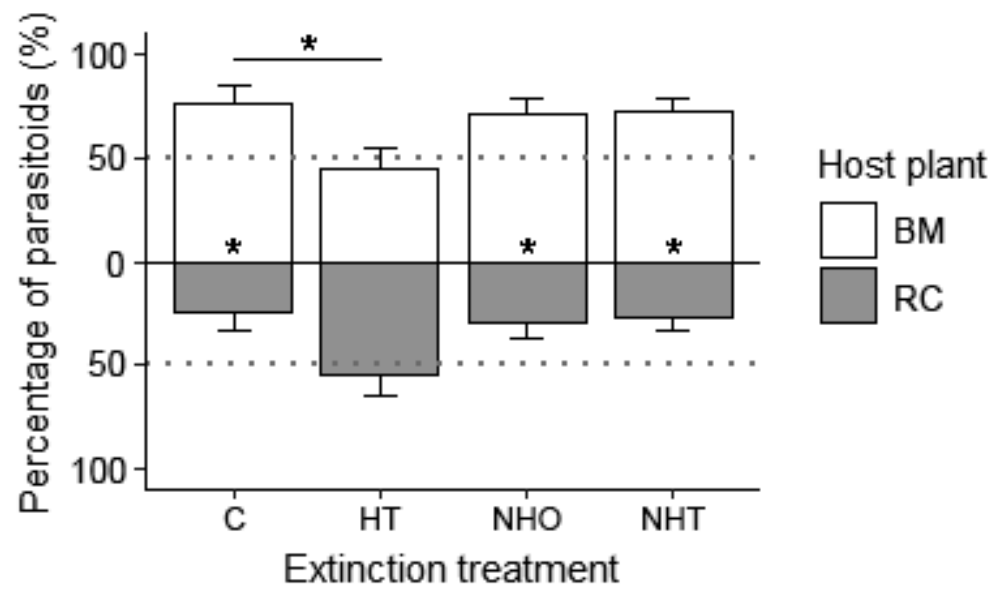

\section{b) Host plant preference after extinction with red cabbage}

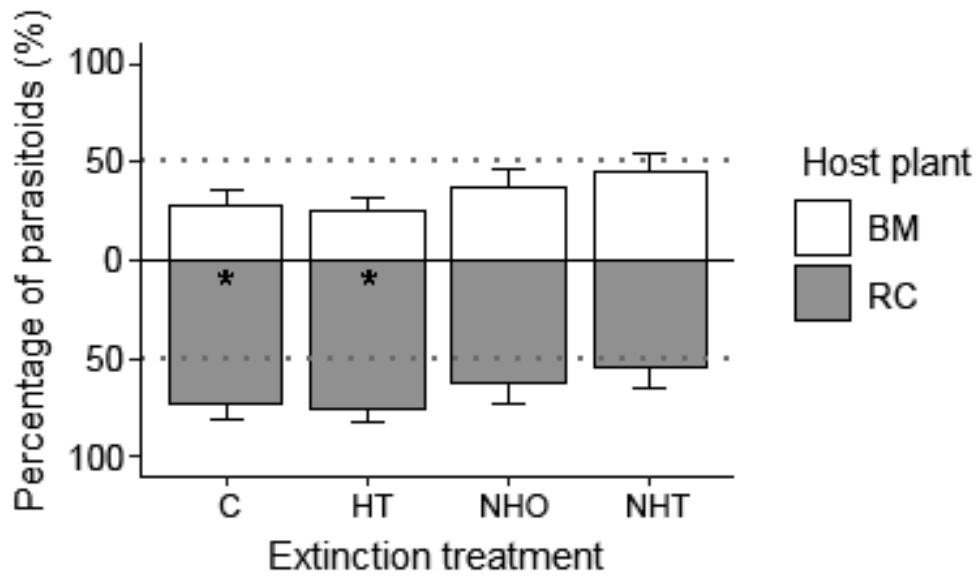

Figure 3. Host plant preference of Cotesia glomerata after an extinction experience following conditioning on either black mustard (a) or red cabbage (b) $(n=9$ per treatment). Parasitoids were given either no extinction experience (control, C), extinction with host traces (HT) or non-host traces (NHT) for 15 minutes, or were given an oviposition in a non-host on a leaf with host traces, after which it was rested for 15 minutes in a clean Petri dish (NHO). A significant difference $(p<0.05)$ between the control (C) and any of the extinction groups is indicated with an asterisk above the bars. Asterisks within bars indicate a significant preference $(p<0.05)$ for a host plant. 
When parasitoids received an extinction experience 10 minutes after conditioning, the conditioned host plant preference was lost $(t=1.267, p=0.241)$, and their preference differed from the control group $(t=2.400, p=0.029)$. One hour after the extinction experience, $C$. glomerata still showed no preference for the conditioned black mustard plant $(t=-0.258, p=0.803)$, although behaviour of the control and extinction groups did not differ significantly $(t=1.904, p=0.075)$. However, 4 hours after the extinction experience, the conditioned preference for the black mustard host plant returned $(t=3.553, p=0.007)$, and behaviour was comparable to the control group $(t=-0.832, p=0.418)$, showing spontaneous recovery.

\section{Extinction and spontaneous recovery of the black mustard host plant preference}
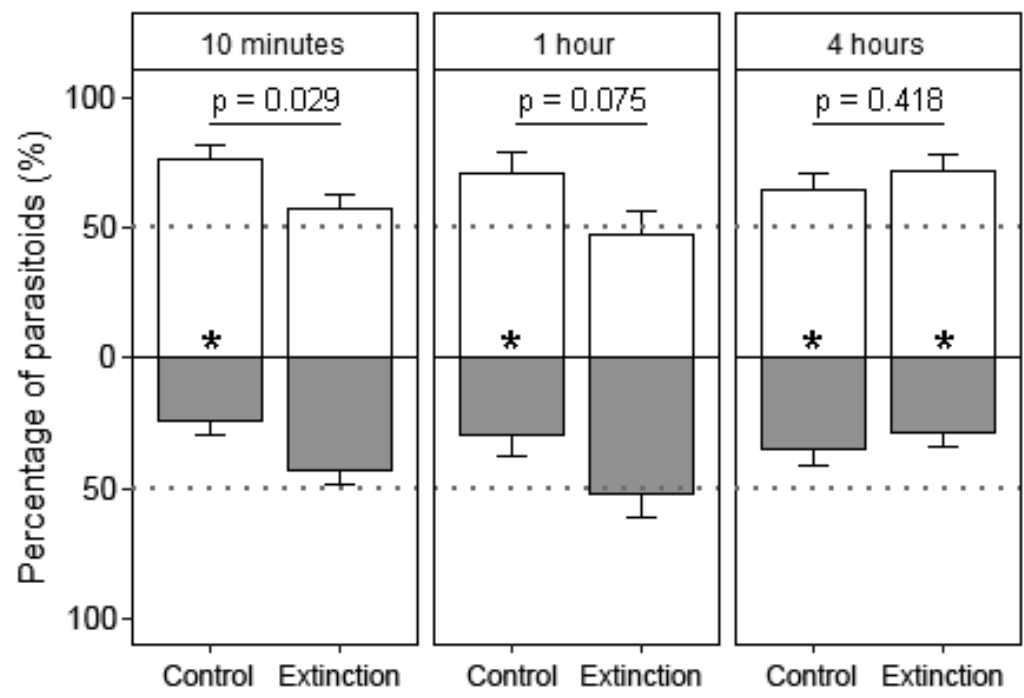

Host plant

Extinction treatment

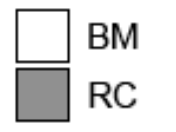

Figure 4. Spontaneous recovery of host plant preference of conditioned C. glomerata after extinction with black mustard. Parasitoids were conditioned on black mustard and then given either no extinction experience (Control) or a 15-minute extinction experience with host traces (Extinction) ( $\mathrm{n}=9$ per treatment). Host plant preference was then tested at different time points; 10 minutes, 1 hour and 4 hours after the extinction experience, to check for extinction and subsequently the spontaneous recovery of the black mustard host plant preference. P-values above bars indicate a significant difference $(p<$ $0.05)$ between control and extinction groups. Asterisks within bars indicate a within-group significant preference $(p<0.05)$ for a host plant. 


\section{Discussion}

Parasitoids need to constantly adapt their behaviour to efficiently locate their hosts in a temporally and spatially dynamic environment. They can store information on local host presence and distribution in their memory (Hoedjes et al., 2011), but this information needs to be re-evaluated upon each encounter of host-associated cues. In this study, we showed that a conditioned host plant preference in Cotesia glomerata can disappear following an extinction experience, and that this conditioned preference can recover over time. Furthermore, we show that the consequences of an extinction experience can both depend on the type of extinction experience and on the host plant species.

Cotesia glomerata has the ability to learn to associate HIPVs with the presence of its host Pieris brassicae after a single oviposition in this host (Geervliet et al., 1998b; Bleeker et al., 2006; Smid et al., 2007), which we have also shown here with black mustard and red cabbage. We subsequently tested how the resulting conditioned host plant preference would be affected by different types of extinction experiences. We hypothesised that an extinction experience with host traces would not lead to the formation of extinction memory, because host traces may still be an indication of host presence and can lead to the formation of a reward memory (Hoedjes et al., 2011). However, parasitoids conditioned on black mustard lost their conditioned preference for this host plant after an extinction experience with host traces for 15 minutes. The conditioned host plant preference spontaneously recovered within 4 hours, which indicates that the retrieval of the reward memory was temporarily blocked by the formation of an extinction memory. Since associated HIPVs and host traces both indicate host presence, parasitoids may have a strong expectation of a host oviposition. Not meeting this expected reward within 15 minutes of host searching may have initiated the formation of the extinction memory, suggesting that alterations in the reliability of host-derived cues can trigger extinction memory formation.

An extinction experience with non-host traces also had the opposite effect of what we expected. We hypothesised that such an experience would trigger the formation of an extinction memory, because encountering non-host traces undermines the reliability of the association between the learned cues and the presence of hosts. However, when parasitoids were given an extinction experience with non-host traces 
of Mamestra brassicae, the conditioned host plant preference did not change. These findings are in line with those described above for host traces, when considering that parasitoids likely have a relatively low expectation to find a host nearby non-host traces. Indeed, non-host traces did not trigger the same excitatory behavioural response in $C$. glomerata as host traces in this study. When the reward expectation is low, a single 15-minute extinction experience is apparently not sufficient to trigger the formation of extinction memory. In this case, the benefits of a change in behaviour might not outweigh the costs of memory formation (Dukas, 1999).

An extinction experience on black mustard, with host traces followed by an oviposition in a non-host, did not lead to a change in the conditioned host plant preference. This suggests that the oviposition in the non-host may cancel out the effect of the encountered host traces. Though speculative, the parasitoid's strong expectation of a host oviposition upon encountering host traces, may have been fulfilled by the non-host oviposition. Either way, these results show that an extinction experience with host-related cues is more likely to cause behavioural alterations than a non-host-related extinction experience. This unchanging behaviour after M. brassicae non-host encounters, has been observed before (Chapter 3 and 4), where $C$. glomerata parasitoids did not stop visiting non-host infested plants, even after ovipositing in non-hosts. The effect of multiple non-host encounters still remains to be investigated, but both in humans and insects, the formation of an extinction memory depends on the number of extinction experiences (Lagasse et al., 2009; Eisenhardt et al., 2013; Exton-McGuinness et al., 2015).

Pieris brassicae caterpillars occur on various brassicaceous plant species in nature (Bell and Muller, 1973; Carter, 1984), and we tested the effect of extinction experience with two of them. Interestingly, we found a plant species-specific effect of an extinction experience with host traces. Parasitoids conditioned on black mustard lost their preference for this host plant after an extinction experience with host traces, while parasitoids conditioned on red cabbage did not. This host plantspecific effect suggest that these plant species may have different consolidation speeds of the reward memory. Conditioning on red cabbage might have led to a faster consolidating reward memory, that was in the MTM phase once the extinction experience was given, hence its stronger resistance to extinction. Prepared learning is known to be host species-specific in C. glomerata (Kruidhof et al., 2012), but these results indicate that they are also plant species-specific. 


\section{Chapter 5}

To our knowledge, this is the first study showing extinction and spontaneous recovery of associated memory with herbivore-induced plant volatiles and a host reward in a parasitoid wasp. Extinction and spontaneous recovery have been demonstrated before in a parasitoid. In Papaj et al. (1994) the parasitoid Leptopilina heterotoma was conditioned on a host-infested mushroom substrate and given an extinction experience on a mushroom substrate without hosts 24 hours after conditioning. The conditioned preference for the mushroom substrate disappeared, and subsequently reappeared within 2 hours. We predicted that memories formed through an association with a host oviposition would be more resistant to an extinction experience than reward memories with synthetic cues and artificial rewards (Kaiser et al., 2003; Lagasse et al., 2009; Eisenhardt et al., 2013), because oviposition-related information can be highly valuable for parasitoid fitness. However, a single extinction experience with an uninfested substrate (Papaj et al., 1994) or host traces can be enough to temporarily suppress the retrieval of the reward memory. This temporary suppression might be adaptive, when the lack of host finding accurately predicts current circumstances, but not future opportunities (Papaj et al., 1994). With the spontaneous recovery of the conditioned host plant preference, parasitoids might still profit from this memory when environments continue to change.

Overall, we conclude that in C. glomerata, an associative memory, formed with HIPVs and a host reward, can be more sensitive to host-related extinction experiences than non-host-related extinction experiences and that the effects of an extinction experience can be plant species-specific. A parasitoid's response to an extinction experience seems to be shaped by its preparedness to learn, which is in turn shaped by its evolutionary history with host and host plant-related cues. These results, provide novel insights on how different types of extinction experiences, related to hosts and non-hosts, lead to changes in parasitoid foraging behaviour in an ecological context and further support the context-dependency of parasitoid foraging behaviour.

\section{Acknowledgements}

The NWO open competition grant nr. 824.14.023 awarded to Louise Vet has supported this study. 




\title{
Chapter 6
}

\section{Non-host oviposition by the parasitoid Cotesia glomerata and its effects on foraging behaviour}

\author{
Jessica A.C. de Bruijn ${ }^{1}$ \\ Louise E.M. Vet ${ }^{1,2}$ \\ Hans M. Smid ${ }^{1}$ \\ Jetske G. de Boer ${ }^{2}$
}

${ }^{1}$ Laboratory of Entomology, Plant Sciences Group, Wageningen University, Wageningen, The Netherlands

${ }^{2}$ Department of Terrestrial Ecology, Netherlands Institute of Ecology (NIOO-KNAW), Wageningen, The Netherlands 


\begin{abstract}
Endoparasitoids share an intimate relationship with their host, because offspring can only develop when they have the ability to overcome internal host defences. Though parasitoids search for host that can support offspring development, they can also encounter unsuitable species, so called non-hosts. Though these non-hosts are generally ignored, parasitoid attacks on non-host species have been reported and sometimes even result in egg deposition. To prevent a further waste of eggs, parasitoids might alter their foraging behaviour, but little is known about whether and how such a non-host oviposition can lead to alterations in parasitoid foraging behaviour. In this study, we investigated the interaction between the endoparasitoid Cotesia glomerata and the presumed non-host caterpillar Mamestra brassicae. In the presence as well as in the absence of Pieris brassicae hosts, we observed $C$. glomerata attacks on $M$. brassicae caterpillars. Eggs were deposited, but encapsulated and killed, confirming that this is truly a non-host. Furthermore, a nonhost oviposition had little effect on the foraging behaviour of $C$. glomerata. Parasitoids did not learn to avoid volatiles encountered during a non-host oviposition and did not alter their behaviour in respect to non-host contacts and ovipositions. $C$. glomerata did become more motivated to forage on a non-host infested leaf, but this is a common phenomenon after a parasitoid's first oviposition. We propose that egg deposition in non-hosts might be due to a high egg load, which makes parasitoids less selective on host quality when they have few reproductive opportunities. As parasitoids continue to forage, they can gain more information about the overall quality of hosts in their environment and may alter their host acceptance strategy accordingly.
\end{abstract}

Key words: Parasitoid-host interactions, Mamestra brassicae, cabbage, herbivoreinduced plant volatiles, learning, egg deposition 


\section{Introduction}

Parasitoids lay one or more eggs in or on the body of another insect, where successful development of their offspring ultimately leads to the death of the host (Godfray, 1994). Parasitoids that deposit eggs inside the host body, i.e. endoparasitoids, share a very intimate relationship with their hosts (Price, 1980). Their host range is generally limited, since they need to overcome internal host defences for successful offspring development (Strand and Obrycki, 1996; Vinson, 1998).

Overcoming host defences may be especially challenging for so-called koinobiont parasitoids that allow their host to continue development after parasitism (Askew and Shaw, 1986), allowing it to also mount a defensive response (Strand and Obrycki, 1996; Kraaijeveld et al., 1998). Whether or not an insect species is a suitable host for a certain parasitoid is partially determined by this defence response to the parasitoid's eggs. Species that are unsuitable as hosts, so-called non-hosts, generally eliminate parasitoid offspring through encapsulation, a process in which haemocytes form an envelope around the offspring, eventually killing them through asphyxiation and the release of necrotizing substances (Strand and Obrycki, 1996; Kraaijeveld et al., 1998). Encapsulation is a very general defence response that is present in most arthropods and related invertebrates (Salazar-Jaramillo et al., 2014). Parasitoids, in turn, can overcome this physiological defence by depositing eggs in locations inaccessible by haemocytes, through molecular mimicry, or by injecting toxins and other substances that temporarily or permanently suppress the host's immune system (Strand and Pech, 1995; Strand and Obrycki, 1996; Kraaijeveld et al., 1998).

Parasitoids predominantly attack hosts that share similar life-history traits and that often occur in the same habitat (Strand and Obrycki, 1996). In a natural environment, these habitats typically consist of a multitude of plant species, harbouring a wide array of herbivorous insects, where it is common that multiple herbivore species feed simultaneously on the same plant individual and even on the same leaf (Vos et al., 2001; Dicke et al., 2009). This can lead to plants that contain a mix of suitable hosts and non-hosts. Godfray (1994) suggested that such non-hosts are generally ignored by parasitoids when co-occurring with a suitable host species and may only be accepted for oviposition when parasitoids are given no other choice. However, various parasitoid species, such as Cotesia glomerata, Aphidius ervi and Praon 


\section{Chapter 6}

pequodorum, were recently reported to frequently attack (presumed) non-hosts, both in the presence and absence of a suitable host species (Meisner et al., 2007; Bukovinszky et al., 2012; Chabaane et al., 2015; de Bruijn et al., 2018b).

Non-hosts can influence parasitoid host searching behaviour during the entire host searching process (Vinson, 1998; de Rijk et al., 2013). Many parasitoids use herbivore-induced plant volatiles (HIPVs) to locate their herbivorous hosts from a distance, because insects are generally inconspicuous and hard to detect (Vet and Dicke, 1992). Although HIPVs can provide information on the identity of the herbivore feeding on a plant, parasitoids cannot always distinguish between volatiles induced by host and non-host insects. For example, Cotesia flavipes does not discriminate between HIPVs of sugarcane infested with the suitable host Diatraea saccharalis or a non-host Spodoptera frugiperda, though both species commonly occur in sugarcane crops (Peñaflor et al., 2017). After arriving on a plant, parasitoids generally assess the proximity of a suitable host species through contact with host frass, silk, honeydew and saliva (Strand and Obrycki, 1996), which can trigger arrestment and probing behaviour (Vinson, 1976). However, the parasitoid Cotesia kariyai also showed arrestment behaviour when contacting traces of Acantholeucania loreyi, which is known as a non-host (Takabayashi et al., 1995). During the final foraging stage, i.e. when a parasitoid stings the host, the female decides whether or not to deposit eggs and how many eggs are laid (Hays and Vinson, 1971; Vinson, 1975; Vinson, 1976; Vinson, 1998). This process of host acceptance is triggered by the presence of certain cues and the absence of deterrents in the host (Vinson, 1998). However, these cues do not always reliably indicate host suitability, since various studies report egg deposition in insects known as non-hosts (Arthur et al., 1969; Arthur et al., 1972; Takasu and Lewis, 2003; Bukovinszky et al., 2012; Chabaane et al., 2015). For example, the ichneumonid parasitoid Itoplectis conquisitor readily attacks and deposits eggs in various Lepidoptera species, including non-host species (Arthur et al., 1969).

Although depositing eggs in a non-host may be maladaptive behaviour, it may allow parasitoids to gain information on their current environment, resulting in changes in subsequent foraging behaviour. Indeed, parasitoids can learn to associate a host oviposition with various environmental cues, such as the HIPVs of the plant on which the host was feeding (Vet et al., 1995; Bleeker et al., 2006; Hoedjes et al., 2011; Takemoto et al., 2011). After this learning experience, the association is stored 
as memory that alters foraging behaviour and can improve foraging efficiency (Papaj and Vet, 1990; Hare et al., 1997; Kruidhof et al., 2015). In contrast to the effects of a host oviposition on parasitoid foraging behaviour, little is known about the influence of a non-host oviposition.

Here, we address non-host oviposition and its effects on foraging behaviour, using the parasitoid Cotesia glomerata and the caterpillar Mamestra brassicae, a presumed non-host species. C. glomerata has been used in many studies on tri-trophic interactions and parasitoid learning, and $M$. brassicae has frequently been used as a non-host (Geervliet et al., 1996; Bukovinszky et al., 2012; de Bruijn et al., 2018b; Vosteen et al., 2019). Interestingly, C. glomerata was previously found to distinguish between HIPVs induced by the suitable host $P$. brassicae and the non-host Spodoptera littoralis (Chabaane et al., 2015), but is strongly attracted to HIPVs of plants infested with M. brassicae (Geervliet et al., 1996; de Rijk et al., 2013; Vosteen et al., 2019) and has been observed to attack and even deposit eggs this species (Bukovinszky et al., 2012; de Bruijn et al., 2018b). Based on these observations, we further investigated the relationship between C. glomerata and M. brassicae. More specifically, we tested the hypotheses that (1) C. glomerata deposits eggs upon attacking $M$. brassicae caterpillars, but these eggs do not survive; (2) C. glomerata can learn to avoid volatiles associated with an oviposition in the non-host $M$. brassicae; and (3) such an oviposition experience subsequently alters C. glomerata's foraging behaviour in the presence and absence of suitable hosts near M. brassicae non-hosts.

\section{Materials and methods}

\section{Insects}

For experiments, $1^{\text {st }}$ instar Pieris brassicae (Lepidoptera: Pieridae) host caterpillars, $1^{\text {st }}$ instar Mamestra brassicae (Lepidoptera: Noctuidae) non-host caterpillars and Cotesia glomerata (Hymenoptera: Braconidae) females of 3-5 days old were used. Brussels sprouts plants (Brassica oleracea var. gemmifera cultivar Cyrus) were used to rear both caterpillar species and for all experiments. Insects were reared at the Laboratory of Entomology, Wageningen University, in a greenhouse with a L16:D8 photoperiod (both natural and artificial light), at $21 \pm 1{ }^{\circ} \mathrm{C}$ and $50-70 \%$ relative 


\section{Chapter 6}

humidity. Each year, insect colonies were re-established with individuals collected from cabbage fields near Wageningen.

Cotesia glomerata females were obtained by transferring cocoons from the laboratory colony to a mesh cage $(30 \times 30 \times 30 \mathrm{~cm}$, Bugdorm- 1 Insect rearing cage, type DP1000, Megaview Science, Taiwan) with water and honey, and placing it in a climate cabinet at $21 \pm 1{ }^{\circ} \mathrm{C}$ and $50-70 \%$ relative humidity. Females of 2 days old were selected from these cages and transferred to a new cage with water and honey, until use in experiments.

\section{Egg deposition and survival}

To test the first hypothesis, we determined whether $C$. glomerata deposits eggs in the presumed non-host M. brassicae, further referred to as Mamestra, and whether these eggs could survive, by dissecting parasitized Mamestra caterpillars. To stimulate parasitoids to oviposit in these non-host, we prepared B. oleracea leaves with fresh host traces, i.e. frass, silk and feeding damage of $P$. brassicae caterpillars further referred to as Pieris caterpillars. A 5-week-old B. oleracea plant was infested with approximately 200 first instar Pieris caterpillars, which were spread over 4 leaves in groups of approximately 50 caterpillars. After 24 hours, these infested leaves were detached from the plant and Pieris caterpillars were removed from the leaves. First instar Mamestra caterpillars were offered on these leaves with fresh traces of the Pieris host, to stimulate oviposition behaviour. A single female parasitoid was released on the leaf with non-hosts and allowed to oviposit in 4 Mamestra caterpillars. Caterpillars were collected directly after a parasitoid was observed to oviposit in it for at least 2 seconds. Attacks that lasted less than 2 seconds were not considered successful and these caterpillars were discarded. Ten groups of 25 parasitized caterpillars were transferred to plastic boxes $(13 \times 17 \times 6.5 \mathrm{~cm})$, where they were provided with 4 fully expanded leaves of 8-week-old B. oleracea plants. Holes were punctured in the plastic lid for ventilation and a layer of absorbent paper was placed under the lid to absorb moisture that condensed on the lid. The plastic boxes were placed in a climate cabinet at $21 \pm 1{ }^{\circ} \mathrm{C}$ and $50-70 \%$ relative humidity. After 0, 24, 48, 72 and 96 hours, 30 caterpillars were randomly selected from these boxes and dissected in a drop of water to count parasitoid eggs. Dissections were done with a small pair of tweezers and an insect pin in a glass Petri dish, using a 
stereomicroscope with backlight illumination. Since encapsulated eggs were assumed to not survive, we only quantified unencapsulated eggs.

\section{Associative learning with a host or non-host oviposition}

For the second hypothesis, we assessed whether an oviposition in the Pieris host caterpillar or Mamestra non-host caterpillar leads to the formation and retention of associative memory 24 hours after the experience, by testing if the parasitoid develops a preference for volatile cues offered during egg deposition. Parasitoids were conditioned using synthetic odours and tested in the high-throughput individual T-maze system with video recording and tracking software, as described in de Bruijn et al. (2018a). In short, parasitoids were given an associative learning experience with a classical conditioning procedure, where they could learn to associated a single Pieris host oviposition experience with either a $4 \%$ vanilla or $4 \%$ coffee odour extract (Nielsen-Massay Vanillas Intl., Leeuwarden, the Netherlands). These extracts were presented as a globular sphere of odorized agarose (A9539-500 g, Sigma) on a micro pestle (SIAL501ZZ0, Sigma-Aldrich), on which Pieris frass and silk were applied. A single parasitoid was collected in a glass vial and brought into contact with the Pieris traces to trigger oviposition behaviour, upon which either a Pieris or Mamestra caterpillar was offered on the tip of a fine paint brush to let the parasitoid oviposit. After an oviposition, parasitoids were placed in a small cage $(17.5 \times 17.5 \times 17.5 \mathrm{~cm}$, Bugdorm-41515 Insect Cage, type BD41515, Megaview Science, Taiwan) with water any honey until testing. Four treatments were used; parasitoids oviposited in either Mamestra or Pieris, on either vanilla or coffee scented agarose. Per treatment, 90 parasitoids were conditioned. This procedure, with a single oviposition in Pieris, is known to result in the formation of associative memory, resulting in a clear preference for the conditioned odour that is maintained for at least 24 hours (de Bruijn et al., 2018a).

Testing for memory retention was done in the high-throughput individual T-maze, consisting of 36 two-choice arenas, offering $2 \%$ vanilla-scented agarose on one side and $1 \%$ coffee-scented agarose on the other side. Per treatment, 9 parasitoids were individually released in each of these 36 two-choice arenas, 24 hours after conditioning, and their behaviour was recorded for 10 minutes. From this recording, we extracted first choice, i.e. whether a parasitoid first walked into the vanilla- or the coffee-scented agarose zone. In each two-choice arena, a vanilla conditioned 
parasitoid and a coffee conditioned parasitoid were tested in two subsequent runs on the same day. Since the ability to retain this information could in theory depend on the number of deposited eggs, we dissected a subset of the Pieris and Mamestra caterpillars. Twenty caterpillars of each species, 10 per conditioning odour, were dissected within 24 hours after the oviposition, to determine the number of parasitoid eggs.

\section{The effect of non-host oviposition experience and host presence on parasitoid foraging behaviour}

For the third hypothesis, we tested how C. glomerata foraged around host and nonhost caterpillars after a non-host oviposition experience in Mamestra (Fig. 1). Parasitoids were either kept unconditioned or were given one Mamestra oviposition experience, hereafter called a Mamestra experience. This Mamestra experience was given by letting a female parasitoid oviposit once in a Mamestra non-host caterpillar on a B. oleracea leaf with host traces in a Petri dish (Fig. 1a). We expected that such an experience would allow the parasitoid to learn to avoid interacting with these nonhosts on the infested leaf. Leaves with host traces were prepared as described above for the egg deposition and survival experiment. Since short-term and long-term effects of a non-host oviposition experience might affect subsequent foraging behaviour differentially, parasitoids were tested either within an hour after the oviposition experience, or on the following day.

Parasitoid foraging behaviour was tested in a round glass Petri dish $(20 \mathrm{~cm}$ diameter, $5 \mathrm{~cm}$ high) with a $B$. oleracea leaf that was infested 24h before, with either 10 Mamestra caterpillars for a no-choice situation, or 5 Mamestra and 5 Pieris caterpillars for a choice situation (Fig. 1b). We infested leaves by placing groups of 5 caterpillars in a clip cage, of which two were attached to a fully expanded leaf of a 5-week-old B. oleracea plant. Just prior to testing, the infested leaf was detached from the plant, its petiole was wrapped in moist cotton wool, and it was placed upside-down in the glass Petri dish. We made sure that 10 caterpillars were present on the leaf and replaced missing caterpillars if needed. 


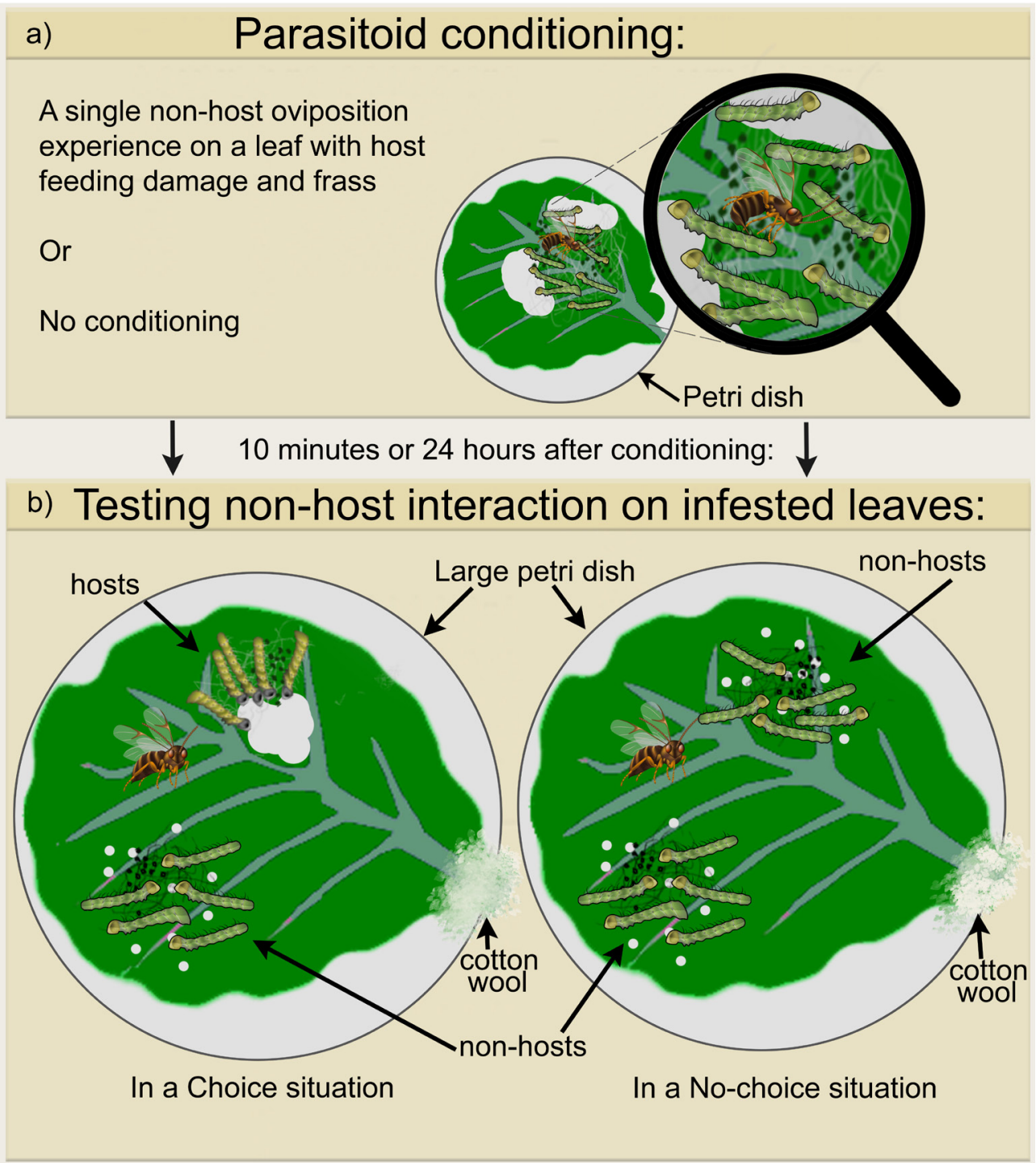

Figure 1. Overview of the experimental procedure to test how Cotesia glomerata parasitoids foraged around Pieris brassicae host and Mamestra brassicae non-host caterpillars after a non-host oviposition experience in a $1^{\text {st }}$ instar $M$. brassicae caterpillar. Parasitoids were conditioned in a Petri dish where they were either allowed to oviposit once in a M. brassicae non-host on a leaf with feeding damage and frass of the host $P$. brassicae, i.e. given a Mamestra experience, or they were kept unconditioned (panel a). Parasitoid foraging behaviour was subsequently tested on a detached Brassica oleracea leaf as depicted in panel $\mathrm{b}$. These leaves were infested 24 hours prior with either 5 host caterpillars and 5 nonhosts caterpillars, representing a choice situation, or with 10 non-hosts, representing a no choice situation. See text for a more detailed description. 
A single parasitoid was then released inside the Petri dish, at the centre of the lid. A hand-held computer (Workabout Pro 3, Zebra Technologies Corp., Illinois, USA) with behaviour recording software (The Observer XT 10, Noldus Information Technology B. V., Wageningen, The Netherlands) was used to record parasitoid foraging behaviour during an observation period of 10 minutes. With this software, we assessed whether a parasitoid responded, i.e. whether it started foraging by contacting the infested leaf, and whether it contacted and subsequently oviposited in the offered host and non-host caterpillars. After 10 minutes, the parasitoid was removed, and caterpillars were replaced if any ovipositions had occurred. A single leaf was re-used 3 times before being discarded, once for each of the 3 corresponding treatments. The order of these treatments was randomized and each of the 6 treatments was replicated 24 times.

\section{Statistics}

All analyses were done in $\mathrm{R}$ version 3.5.0. Egg survival over time, i.e. the number of eggs at different time points, was tested with a zero-inflated generalized linear model with a negative binomial distribution, using R package pscl (Jackman et al., 2007). In the experiment on associative learning, Performance Index (PI) scores of binomial first choice data were used as indices of memory retention as described in de Bruijn et al. (2018a). PI scores were assigned by combining the first choice of a vanilla and a coffee conditioned parasitoid that were tested in the same two-choice arena, in two subsequent runs. If both parasitoids chose the odour on which they were conditioned, a PI of 100 was assigned, one correct choice resulted in a PI of 0 , and if neither parasitoid made a choice for their conditioned odour, a PI of -100 was assigned. If one or both parasitoids did not make a choice within 10 minutes, no PI was formed for this particular pair and the data of the two-choice arena was discarded. One-sample Wilcoxon signed rank tests were used to test if the PI scores of parasitoids that oviposited in Mamestra, and those that oviposited in Pieris, significantly deviated from $0(\mu=0)$, to evaluate the null hypothesis of no memory retention. The number of eggs deposited in Mamestra and Pieris was compared with a Mann-Whitney test. In this test, parasitoids of the two conditioning odours were grouped, since conditioning odour did not affect the number of eggs deposited (Mann-Whitney test, $W=153, p=0.207$ ). 
The effects of oviposition experience and host presence on foraging behaviour were assessed in full factorial generalized linear models with binomial distributions. Separate models were used for each response variable: parasitoid response (the number of parasitoids that started foraging out of total number of parasitoids tested), caterpillar contact (the number of parasitoids that contacted a caterpillar out of those that started foraging) and oviposition (the number of parasitoids that accepted a caterpillar for oviposition out of those that contacted a caterpillar). Experience (Mamestra experience vs. unconditioned) and time after non-host oviposition (10 minutes vs. 24 hours) could not be included in the same models, because unconditioned parasitoids had no time after non-host oviposition, resulting in an incomplete factorial design. We therefore first pooled data of all experienced parasitoids to compare them with unconditioned parasitoids in a first set of overall models. In these models, foraging behaviour was analysed in the two foraging situations by testing the effects of situation (choice vs. no-choice) and experience (Mamestra experience vs. unconditioned) as fixed factors on the three foraging parameters. Caterpillar species-specific foraging behaviour (contact and oviposition) was analysed in the choice situation with caterpillar species and experience as fixed factors. Paired data were used in this case, with parasitoid identity as a random factor, since each parasitoid could contact and accept both caterpillar species for oviposition. Mamestra-specific behaviour was further analysed by testing the effects of situation and experience on caterpillar oviposition (because of unequal numbers of Mamestra caterpillars in the two foraging situations the effects on caterpillar contact could not be tested).

In the second set of time models, the effect of time after non-host oviposition was tested in each of the three types of models described above, using only data of parasitoids with Mamestra experience and replacing experience by time after oviposition (10 minutes vs. 24 hours) as a fixed factor. For all models, a least-square means post-hoc comparison with a Bonferroni error correction was used to test for pairwise differences between groups (Lenth, 2016). 
Chapter 6

\section{Results}

\section{Egg deposition and survival in M. brassicae}

After C. glomerata stung Mamestra (M. brassicae), $90 \%$ of the caterpillars contained parasitoid eggs. The number of eggs decreased rapidly during the first 72 hours and after 96 hours there were no unencapsulated egg left (Fig. 2). This shows that $M$. brassicae has the ability to encapsulate and kill all deposited eggs and is indeed an unsuitable species for offspring development of $C$. glomerata, i.e. a non-host.

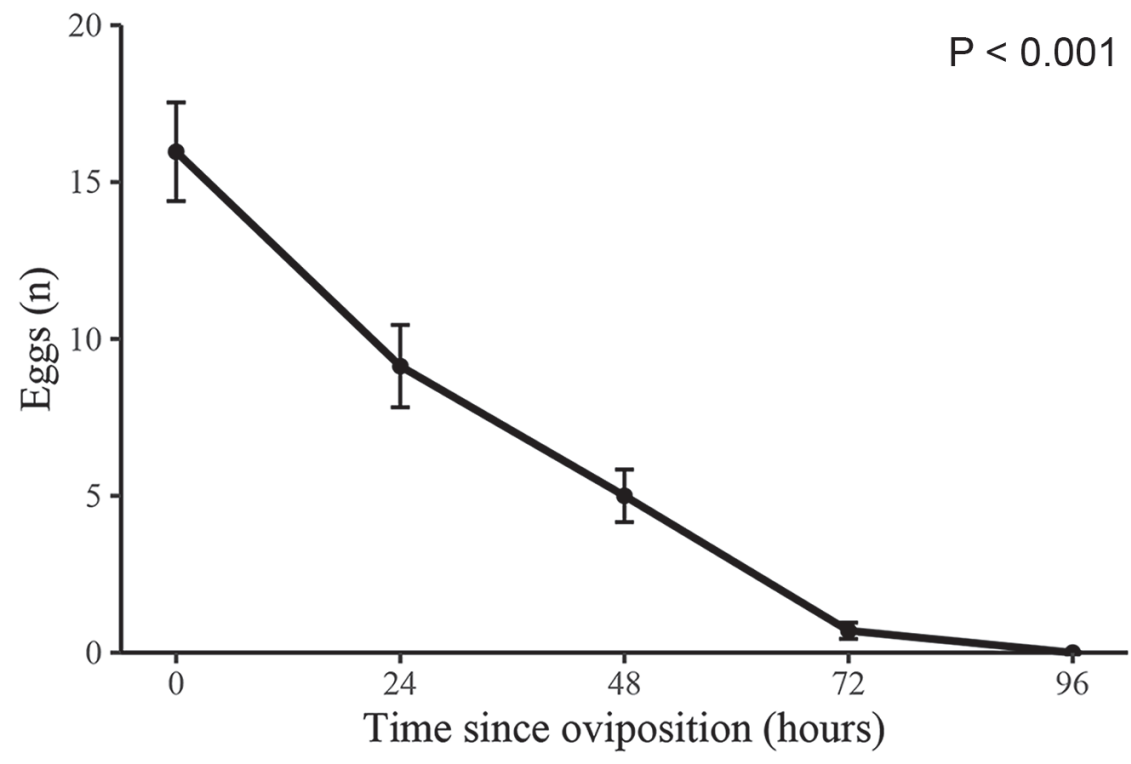

Figure 2. Egg survival of Cotesia glomerata in $1^{\text {st }}$ instar Mamestra brassicae caterpillars at different time points after oviposition. The P-value is based on a negative binomial zero-inflated GLM and indicates a significant difference in egg numbers over time. Per time point 30 caterpillars were dissected and error bars depict the standard error of the mean.

\section{Associative learning with a host or non-host oviposition}

An oviposition in a Pieris host caterpillar ( $P$. brassicae) resulted in significant memory retention, as demonstrated by a clear preference of $C$. glomerata for the conditioned odour $(V=360, p<0.001$, Fig. 3). In contrast to our expectation, 
oviposition in the non-host Mamestra did not result in memory retention after 24 hours $(V=388.5, p=0.322)$ and parasitoids were neither attracted, nor repulsed, by the odours. Furthermore, memory retention did not depend on the number of deposited eggs, since egg numbers in Pieris and Mamestra caterpillars did not differ ( $W=141, p=0.112$ ). In Pieris $95 \%$ of the caterpillars contained parasitoid eggs and $21.4 \pm 2.0$ eggs were laid. Oviposition in Mamestra led to egg deposition in $90 \%$ of the caterpillars, with $18.4 \pm 1.8$ eggs per oviposition.

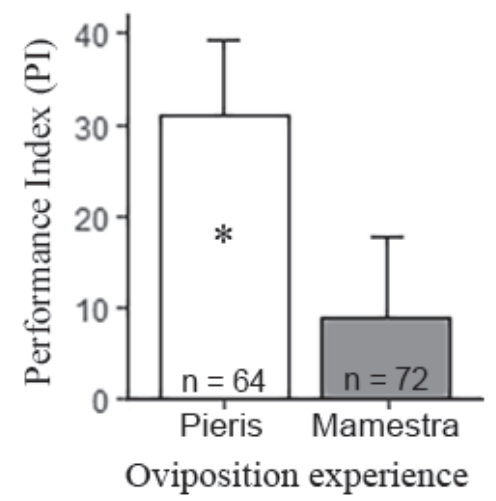

Figure 3. Memory retention 24 hour after associative learning with an oviposition in either $P$. brassicae (Pieris) or M. brassicae (Mamestra). Sample sizes (n) and significant memory retention $\left(^{*}\right)$ are depicted within the bars.

\section{The effect of non-host oviposition experience and host presence on parasitoid foraging behaviour}

Parasitoid response, i.e. the percentage of females that started foraging on the infested leaf, depended on whether they had been given prior non-host oviposition experience and on the situation in which they foraged (Interaction $X^{2}=4.514, p=$ 0.034, Fig. 4A and supplementary table 1). Parasitoids with a Mamestra experience were more motivated to search the infested leaf in the no-choice situation than unconditioned parasitoids, but this was not the case in the choice situation. The percentage of parasitoids contacting a caterpillar (either Pieris or Mamestra) was significantly higher when hosts were present $\left(X^{2}=23.768, p<0.001\right)$, but was not influenced by experience $\left(X^{2}=0.447, p=0.504\right)$, nor by the interaction $\left(X^{2}=1.031\right.$, $p=0.310)$. The same results were found for oviposition: a higher percentage of 
parasitoids accepted a caterpillar for oviposition in the choice situation $\left(X^{2}=27.180\right.$, $p<0.001)$, while experience $\left(X^{2}=0.375, p=0.540\right)$ and the interaction term $\left(X^{2}=\right.$ $1.053, p=0.305)$ had no significant effect.

Parasitoid foraging behaviour was caterpillar species-specific (Supplementary table 2). In the choice situation, parasitoids were more likely to contact Pieris caterpillars than Mamestra caterpillars $\left(X^{2}=14.966, p<0.001\right.$, Fig. 4B). With respect to parasitoid experience, parasitoids with Mamestra experience were just as likely to contact a caterpillar as unconditioned parasitoids, but this effect was marginally insignificant $\left(X^{2}=3.336, p=0.068\right)$. Contact with a Pieris caterpillar led to oviposition in $96-100 \%$ of the parasitoids, while significantly fewer, yet still 50 $71 \%$, of the parasitoids accepted Mamestra non-hosts for oviposition $\left(X^{2}=27.43, p\right.$ $<0.001$, Fig. 4C). We observed this effect in both experience groups, but it was less clear for unconditioned parasitoids, because only a few unconditioned parasitoids contacted Mamestra. Analysis of Mamestra-specific behaviour (supplementary table 3), showed that up to $71 \%$ of the parasitoids oviposited in Mamestra (Fig. 4D) and that more parasitoids oviposited in the non-host in the choice situation than in the no-choice situation $\left(X^{2}=3.859, p=0.049\right)$. Time after Mamestra experience (10 minutes or 24 hours) did not significantly influence any of the foraging parameters. In this second set of models, the effects of foraging situation and caterpillar species on the three foraging parameters were similar as in the first set of models described above. Furthermore, the interactions with time after Mamestra experience were never significant (Supplementary tables 4-6). Parasitoids with a Mamestra oviposition were highly responsive to the infested leaf, both after 10 minutes $(91 \%$ in the choice situation, $96 \%$ in the no-choice situation) and after 24 hours $(87 \%$ in the choice situation, $92 \%$ in the no choice situation). 


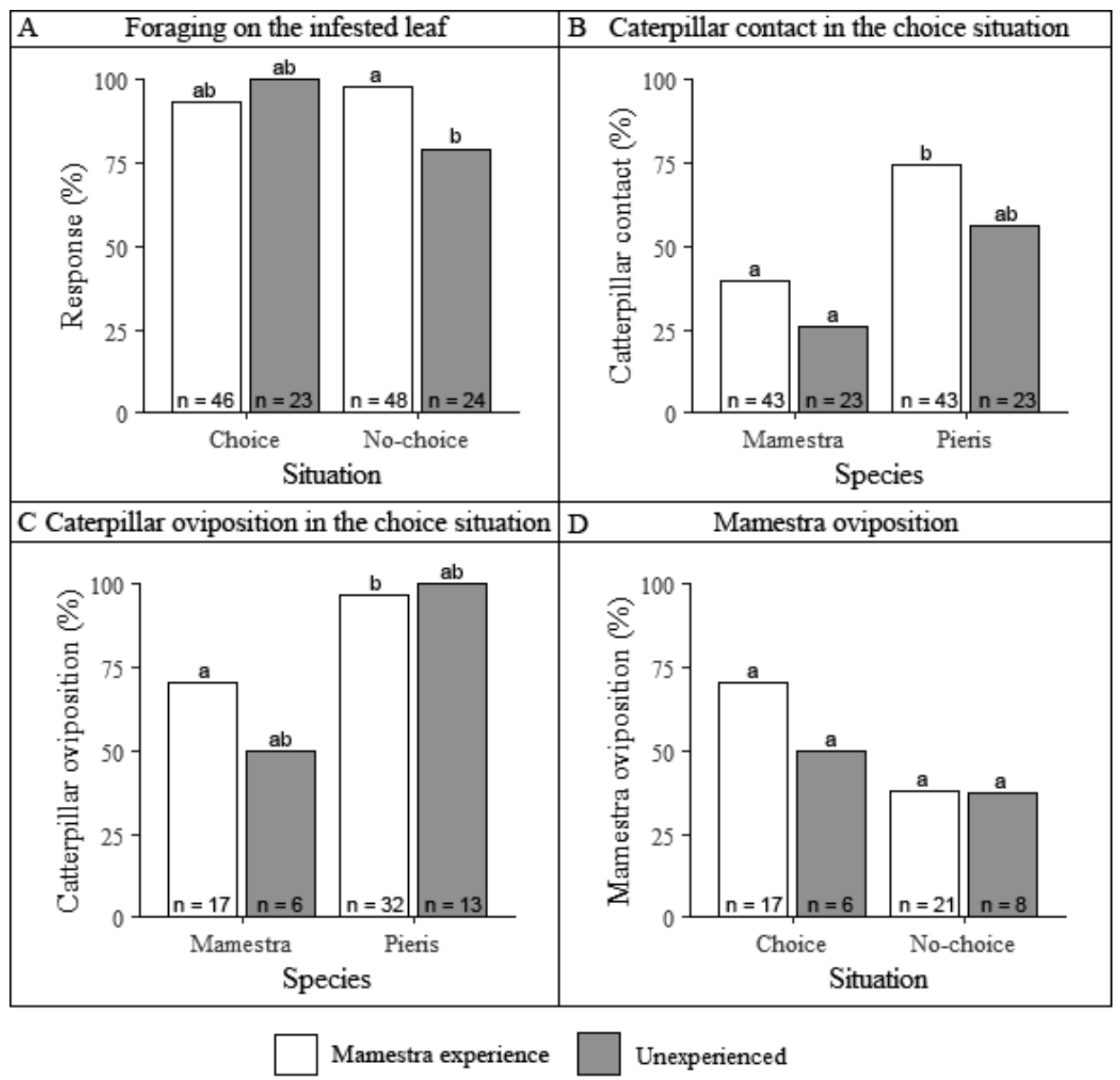

Figure 4. Parasitoid behaviour on a caterpillar infested Brassica oleracea leaf. The percentage of parasitoids with either no experience (Unconditioned, white) or a Mamestra oviposition experience (Mamestra experience, grey) that, (A) started foraging on the infested leaf with Mamestra caterpillars (no-choice situation) or with both Mamestra and Pieris caterpillars (choice situation), (B) made contact with either the Mamestra or Pieris caterpillars and (C) subsequently oviposited within these caterpillar species in the choice situation, and (D) oviposited in Mamestra in the choice and no-choice foraging situations. Different letters per panel indicate significant differences between groups $(p<0.05)$, $\mathrm{n}$ values with the bars represent the sample size. 


\section{Discussion}

In this study, we investigated the interaction between the endoparasitoid Cotesia glomerata and the presumed non-host Mamestra brassicae. The hypothesis that C. glomerata deposits eggs upon attacking $M$. brassicae caterpillars and that these deposited eggs are killed by $M$. brassicae was sustained. C. glomerata generally deposited eggs when attacking $M$. brassicae and clutch sizes were comparable to those in the host Pieris brassicae. Furthermore, we show that M. brassicae has the ability to kill eggs deposited by $C$. glomerata. Even though $M$. brassicae is clearly unsuitable for parasitoid development, up to $71 \%$ of parasitoids oviposited in this non-host. We could not sustain our other two hypotheses, since $C$. glomerata showed little to no change in its foraging behaviour after a non-host oviposition. Parasitoids did not learn to avoid volatiles encountered during a non-host oviposition and they did not alter their behaviour with respect to non-host contact and oviposition. However, after a non-host oviposition, parasitoids did become more motivated to forage on an infested leaf with only non-hosts. This increased motivation to forage after an oviposition in the non-host lasted for at least 24 hours. Apparently, a single oviposition in an unsuitable host species is not stored in memory as an adverse event, even though it leads to a waste of eggs.

To test the first hypothesis, we determined whether $C$. glomerata deposits eggs in the presumed non-host $M$. brassicae and whether these eggs could survive. Before our study, the status of M. brassicae as a non-host was unsure due to observations of oviposition behaviour and egg deposition by C. glomerata (Bukovinszky et al., 2012; de Bruijn et al., 2018b). Here, we confirm that $M$. brassicae is truly a non-host for C. glomerata. Despite egg deposition by $90 \%$ of parasitoids, M. brassicae was able to encapsulate and kill deposited eggs. Surprisingly, clutch sizes of $C$. glomerata in the non-host $M$. brassicae were comparable to those in the host $P$. brassicae. These observations conflict with the general idea that parasitoids do not deposit eggs in non-host species or that they adjust their egg numbers (Vinson, 1975; Vinson, 1976). Egg deposition in non-hosts has, however, been shown in other studies as well (Arthur et al., 1969; Takasu and Lewis, 2003; Bukovinszky et al., 2012; Chabaane et al., 2015). Our findings corroborate those of Chabaane et al. (2015), who found that $C$. glomerata deposits similar clutch sizes in the non-host $S$. littoralis and the host $P$. brassicae, but conflict with observations of Bukovinszky et al. (2012), who found that $C$. glomerata deposits a smaller clutch in the non-host $M$. brassicae than 
in the host $P$. rapae. Since Bukovinszky et al. (2012) did not specify after how much time caterpillars were dissected, it is possible that most of the deposited eggs were already killed by $M$. brassicae.

In the second hypothesis, we predicted that a non-host oviposition would be an aversive experience, allowing parasitoids to learn to avoid this situation during subsequent foraging, but our findings do not support this hypothesis. We observed no memory retention for the conditioned odour 24 hours after a non-host oviposition, while an oviposition in the host $P$. brassicae did result in memory retention, due to its high reward value (Kruidhof et al., 2012). The absence of associative learning after conditioning with a non-host oviposition, in combination with vanilla odour and host frass, has also been found in the parasitoid Microplitis croceipes (Takasu and Lewis, 2003). Although a single oviposition experience in M. brassicae did not lead to behavioural changes in C. glomerata, it is possible that multiple experiences spaced in time will. Repeated ovipositions in hosts are known to lead to more persistent memory (Hoedjes et al., 2011), but further research should confirm if this is also the case with non-host ovipositions.

Interestingly, our study and the study of Takasu \& Lewis (2003) show that parasitoids do detect that non-hosts are unsuitable when ovipositing in them, since in both cases egg are deposited, but no memory is formed. Triggers for egg release and memory formation are apparently different. Triggers for egg deposition are generally amino acids and sugars found in the haemolymph (Arthur et al., 1969; Arthur et al., 1972). Triggers for memory formation are also expected to be present in the haemolymph (Takasu and Lewis, 2003), but the compounds causing this trigger are still unknown and requires more research. Furthermore, results from this study, one of our previous studies (chapter 5) and those of Takasu \& Lewis (2003), indicate that associative learning with host frass can be suppressed by a non-host oviposition. Exposure to host frass alone leads to memory retention after 24 hours in C. glomerata (Geervliet et al., 1998b), but we found no memory retention when this exposure was followed by a non-host oviposition. The same was found by Takasu $\&$ Lewis (2003), where the flight response of $M$. croceipes to a conditioned odour was reduced when exposure to frass was followed by a non-host oviposition.

In the third hypothesis, we expected $C$. glomerata to alter its foraging behaviour after a non-host oviposition, both in the presence and absence of suitable hosts near $M$. 
brassicae non-hosts. However, our results show very little support for this hypothesis. $C$. glomerata did not alter its behaviour in respect to non-host contact and oviposition. However, C. glomerata females were more motivated to forage on an infested leaf with non-hosts than unconditioned females, both 10 minutes and 24 hours after a non-host oviposition experience. This increased motivation is most likely a general phenomenon caused by a parasitoid's first oviposition and unrelated to the caterpillar species. Exposure to hosts and/or their traces can increase the motivational state of parasitoids (Papaj and Prokopy, 1989; McAuslane et al., 1991; Takasu and Lewis, 2003; Bleeker et al., 2006). Our results also show that a high proportion of C. glomerata parasitoids oviposited in M. brassicae in the presence of P. brassicae, even though Godfray (1994) suggested that oviposition in non-hosts would only occur in the absence of suitable hosts. Non-host oviposition was even higher in presence of hosts than in the absence of hosts, corroborating recent studies that also showed frequent non-host acceptance in the presence of a suitable host (Meisner et al., 2007; Bukovinszky et al., 2012; Chabaane et al., 2015). Nevertheless, the non-host oviposition behaviour we observe is also not specific to situations were hosts and non-hosts co-occur, since $38 \%$ of the parasitoids attacked the non-host in the absence of hosts.

Based on our observations, we cannot yet answer the question why parasitoids oviposit in non-hosts and whether this behaviour is specific for C. glomerata. We propose that egg deposition in a non-host species could be associated with high egg loads. Parasitoids tend to adapt their oviposition strategy according to the trade-off between current and future reproductive opportunities, where a high egg load indicates few reproductive opportunities and can lead to the acceptance of hosts of lower quality, such as already parasitized hosts, and even non-hosts (Godfray, 1994; Heimpel and Casas, 2008). The waste of resources might outweigh the costs of retaining and maintaining high numbers of eggs in such cases. C. glomerata is known to have a very high fecundity, it emerges with 500-800 eggs, continues to produce eggs throughout its lifetime and when females are not allowed to oviposit, they can hold up to 2,000 eggs within 2-11 days (Le Masurier, 1991). In our experiment, but also in the studies of Takasu et al. (2003) and Chabaane et al. (2015), well-fed mated parasitoid females of several days old were used, which likely had a high egg load and may therefore have been very willing to deposit some eggs, even in a non-host. Future research should elucidate whether non-hosts are also accepted when parasitoids have a low egg load and whether this phenomenon occurs in parasitoid 
species that have a lower fecundity. If egg deposition in M. brassicae is simply because $C$. glomerata becomes less selective due to an accumulation of eggs, this may also explain why a single non-host oviposition did not result in memory retention in C. glomerata and why it had little effect on its foraging behaviour. With the trade-offs described above, the benefits gained by (long-term) storage of this information might not outweigh the costs of wasting time and eggs, since information storage is known to be a costly process (Dukas, 1999). An alternative, or additional, explanation might be that the observed interactions between $C$. glomerata and $M$. brassicae represent only a snap-shot in coevolutionary time, and hence the evolutionary arms-race, between the two species. Although we can only speculate, it is possible that $M$. brassicae was perhaps once a suitable host, but has evolved the ability to defend itself against $C$. glomerata. Vice versa, it is also possible that non-host oviposition behaviour in C. glomerata can lead to an expansion of its host range over evolutionary time when the parasitoid evolves the ability to overcome host defence.

Parasitoid foraging behaviour is known to be highly context-dependent (Heimpel and Casas, 2008) and is shaped by what parasitoids encounter within their habitat (Vet et al., 1995). Although many parasitoid species are known to be specifically attracted to plants on which suitable host species occur (Vet et al., 1995; Geervliet et al., 1996), these plants are often also used by non-hosts (Vos et al., 2001; Dicke et al., 2009), making non-host encounters inevitable events in nature. Acceptance of these non-hosts might largely depend on the quality of alternative host species in the environment and the expectation of current and future reproductive opportunities (Godfray, 1994). Egg deposition in non-hosts might then be due to a high egg load, which makes parasitoids less selective on host quality when they have few reproductive opportunities. Though this leads to a waste of eggs, parasitoids are expected to alter their host acceptance strategy as they gain information about overall host quality from their environment. 


\section{Supplementary information}

Model overviews of the effect of non-host oviposition experience and host presence on foraging behaviour.

Table 1. Statistical overview of the first set of overall glm models, with the binary response variables response, caterpillar contact and caterpillar oviposition, the fixed factors situation (choice and nochoice) and experience (non-host oviposition experience, unconditioned) and an interaction between these two fixed factors.

\begin{tabular}{|l|l|l|l|}
\hline Response variable & Fixed factors & $X^{2}$-value & P-value \\
\hline & & & \\
\hline Response & Situation*Experience & 4.514 & 0.034 \\
\hline & Situation & 0.647 & 0.421 \\
\hline & Experience & 2.554 & 0.110 \\
\hline & & & \\
\hline Caterpillar contact & Situation*Experience & 1.031 & 0.310 \\
\hline & Situation & 23.768 & $<0.001$ \\
\hline & Experience & 0.447 & 0.504 \\
\hline & & & \\
\hline Caterpillar acceptance & Situation*Experience & 1.053 & 0.305 \\
\hline & Situation & 27.180 & $<0.001$ \\
\hline & Experience & 0.375 & 0.540 \\
\hline
\end{tabular}

Table 2. Statistical overview of the first set of caterpillar species-specific glmer models with the binary response variables caterpillar contact and caterpillar oviposition, the fixed factors caterpillar species (Mamestra or Pieris) and experience (non-host oviposition experience, unconditioned), an interaction between these two factors and parasitoid ID as random factor.

\begin{tabular}{|l|l|l|l|}
\hline Response variable & Fixed factors & $X^{2}$-value & P-value \\
\hline & & & \\
\hline Caterpillar contact & Caterpillar species * Experience & 0.057 & 0.811 \\
\hline & Caterpillar species & 14.966 & $<0.001$ \\
\hline & Experience & 3.336 & 0.068 \\
\hline & & & \\
\hline Caterpillar acceptance & Caterpillar species * Experience & 0.670 & 0.413 \\
\hline & Caterpillar species & 27.430 & $<0.001$ \\
\hline & Experience & 0 & 1 \\
\hline
\end{tabular}


Table 3. Statistical overview of the first set of models, the Mamestra-specific glm model with the binary response variable Mamestra acceptance, the fixed factors situation (choice and no-choice) and experience (non-host oviposition experience, unconditioned), an interaction between these two factors. Only caterpillar acceptance was included, since there was an unequal number of caterpillars to contact in the two foraging situations.

\begin{tabular}{|l|l|l|l|}
\hline Response variable & Fixed factors & $X^{2}$-value & P-value \\
\hline & & & \\
\hline Caterpillar acceptance & Situation*Experience & 0.426 & 0.514 \\
\hline & Situation & 3.859 & 0.049 \\
\hline & Experience & 0.392 & 0.531 \\
\hline
\end{tabular}

Table 4. Statistical overview of the second set of overall glm models, with the binary response variables response, caterpillar contact and caterpillar oviposition, the fixed factors situation (choice and nochoice) and test timing after the non-host oviposition experience (NH experience timing, 10 minutes, 24 hours) and an interaction between these two fixed factors.

\begin{tabular}{|l|l|l|l|}
\hline Response variable & Fixed factors/interaction & $X^{2}$-value & P-value \\
\hline & & & \\
\hline Response & Situation * NH experience timing & 0.032 & 0.858 \\
\hline & Situation & 0.653 & 0.419 \\
\hline & NH experience timing & 0.552 & 0.458 \\
\hline & & & \\
\hline Caterpillar contact & Situation * NH experience timing & 0.198 & 0.657 \\
\hline & Situation & 20.374 & $<0.001$ \\
\hline & NH experience timing & 0.007 & 0.934 \\
\hline & & & \\
\hline Caterpillar acceptance & Situation * NH experience timing & 0.321 & 0.571 \\
\hline & Situation & 23.318 & $<0.001$ \\
\hline & NH experience timing & 0.835 & 0.361 \\
\hline
\end{tabular}




\section{Chapter 6}

Table 5. Statistical overview of the second set of caterpillar species-specific glmer models, with the binary response variables caterpillar contact and caterpillar oviposition, the fixed factors caterpillar species (Mamestra or Pieris) and test timing after the non-host oviposition experience (NH experience timing, 10 minutes, 24 hours), an interaction between these two factors and parasitoid ID as random factor.

\begin{tabular}{|l|l|l|l|}
\hline Response variable & Fixed factors/interaction & $X^{2}$-value & P-value \\
\hline Caterpillar contact & $\begin{array}{l}\text { Caterpillar species * NH experience } \\
\text { timing }\end{array}$ & 0.102 & 0.749 \\
\hline & Caterpillar species & 10.926 & $<0.001$ \\
\hline & NH experience timing & 0.001 & 0.974 \\
\hline & $\begin{array}{l}\text { Caterpillar species * NH experience } \\
\text { timing }\end{array}$ & 0.675 & 0.411 \\
\hline & Caterpillar species & 20.103 & $<0.001$ \\
\hline & NH experience timing & 0.039 & 0.844 \\
\hline
\end{tabular}

Table 6. Statistical overview of the second set of models, the Mamestra-specific glm model, with the binary response variable Mamestra acceptance, the fixed factors situation (choice and no-choice) and test timing after the non-host oviposition experience ( $\mathrm{NH}$ experience timing, 10 minutes, 24 hours) and an interaction between these two factors. Only caterpillar acceptance was included, since there was an unequal number of caterpillars to contact in the two foraging situations.

\begin{tabular}{|l|l|l|l|}
\hline Response variable & Fixed factors/interaction & $X^{2}$-value & P-value \\
\hline & & & \\
\hline Caterpillar acceptance & Situation * NH experience timing & 1.494 & 0.222 \\
\hline & Situation & 4.067 & 0.044 \\
\hline & NH experience timing & 0.140 & 0.708 \\
\hline
\end{tabular}






\section{Chapter 7}

General discussion 

Parasitoid wasps lay their eggs in or on other organisms, which thereafter function as a host for the developing offspring (Godfray, 1994). To reproduce, parasitoid females have to find their inconspicuous hosts in complex habitats with diverse plant and herbivore communities that vary in time and space (Vet et al., 1995; Wajnberg, 2006; Dicke et al., 2009). Parasitoid foraging behaviour for hosts is under strong selection pressure, since it is directly linked to offspring production, and therefore to fitness. This makes them ideal organisms in studies on foraging theory (van Baalen and Hemerik, 2008; Thiel and Hoffmeister, 2009). Foraging in dynamic environments requires complex decisions, where parasitoids acquire, process, store and use information related to the availability and distribution of hosts in their current environment to increase their host encounter rate (Turlings et al., 1993; Vet et al., 1995). Such information can consist of olfactory, visual and tactile cues that originate from the host itself, or, in the case of herbivorous hosts, from the plant on which the host feeds. Host-derived cues, such as frass, silk, honeydew and other body secretions, are generally reliable indicators of host presence, but they are detectable only in the direct vicinity of the host. Therefore, parasitoids commonly rely on environmental cues that indirectly indicate host presence, such as herbivoreinduced plant volatiles (HIPVs) (Vet et al., 1995).

HIPVs are less reliable in predicting host presence, but parasitoids can verify their reliability when encountering these HIPV producing plants in the local environment. Parasitoids can associate HIPVs with an oviposition in a host and store this information as an associative memory, which can improve a parasitoid's foraging efficiency (Papaj and Vet, 1990; Vet et al., 1995; Hare et al., 1997; Kruidhof et al., 2015). These memories can vary in their temporal stability, i.e. their persistence. Short-term memory is formed directly after an experience, but fades quickly. More persistent, i.e. longer lasting, memory forms, such as long-term memory, can be formed when the value (e.g. host quality) or the encounter frequency of the experience is high (Hoedjes et al., 2011; Smid and Vet, 2016). Such memories allow parasitoids to continuously adjust their behaviour according to their experiences in the local environment. The ease with which a certain association is formed and the temporal dynamics of the formed memory, depend on an insect's preparedness to learn (Smid and Vet, 2016). This prepared learning, is shaped in such a way that it is adaptive in respect to the parasitoid's ecology. 


\section{Chapter 7}

Associated HIPVs may, however, no longer reliably predict host presence, when they lead parasitoids to plants where hosts have developed into a stage that is unsuitable for parasitism, hosts have left the plant or where non-host instead of host herbivores are present (Meisner et al., 2007; de Rijk et al., 2013; Peñaflor et al., 2017). Such unrewarding experiences are called extinction experiences and indicate that previously obtained information has become unreliable, which can have fitness consequences when parasitoids waste time on these plants (Vos et al., 2001). Furthermore, parasitoids may change their behaviour after such extinction experiences, by temporarily or permanently altering their response to the associated environmental cues, to maintain their foraging efficiency.

In this thesis, I developed and used advanced observation set-ups and methods to study how unreliable associative memory, and interactions with Mamestra brassicae non-hosts, shaped the foraging behaviour of the parasitoid Cotesia glomerata. I formulated and tested the following hypotheses:

1) Unreliable associative memory reduces parasitoid foraging efficiency

2) Non-host-related extinction experiences alter a parasitoid's response to associated cues

3) Parasitoids change their foraging behaviour after a non-host oviposition

The validity of these hypotheses was experimentally tested in previous chapters and in this final chapter, I integrate the results from these studies to discuss how parasitoid foraging behaviour is shaped by information-reliability and non-host interactions. I will start by presenting an overview of measuring parasitoid foraging behaviour in the laboratory and how this can be extended to field studies. Next, I discuss the hypotheses introduced above, the context-dependency of parasitoid foraging behaviour and how parasitoid learning can benefit biological control efforts. Finally, I summarize the main conclusions of my thesis and present possible future research perspectives.

\section{Measuring parasitoid foraging behaviour}

Parasitoid foraging behaviour is complex and measuring it for quantitative analysis is challenging due to their small size and high mobility. Although various methods 
exist to measure parasitoid foraging behaviour in the laboratory, most of them can measure only one or a few behavioural parameters due to constraints on time and design (Bitterman et al., 1983; Geervliet et al., 1994; Wäckers, 1994; Huigens et al., 2009; Hoedjes et al., 2012). For example, a wind tunnel can be used to observe how parasitoids forage on and around plants, but every behaviour a parasitoid displays has to be manually recorded. Due to time constraints, it is impossible to record every detail of the foraging behaviour and therefore only specific behaviours are recorded. In field studies this is even more challenging, because individuals are difficult to locate and track in more complex and spacious environments. Therefore, behaviour is generally only measured with endpoint parameters, such as host parasitism levels (Kruidhof et al., 2015; De Rijk et al., 2018). Such measurements provide little insight into what happens during the foraging process, and how parasitoids interact with their environment. More advanced methods, with cameras, computers and various software applications, have been developed in recent years that allow for the measurement of detailed insect foraging behaviour in the laboratory and field (Manoukis and Collier, 2019). Manual event recorders of animal behaviours exist already for a long time (Celhoffer et al., 1977), but have become more advanced in recent years. With The Observer ${ }^{\circledR}$ XT (Zimmerman et al., 2009) for example, various individual behaviours of an insect can easily be scored, collected and processed (Chapter 3). Furthermore, automated video tracking software, such as Buritrack (Colomb et al., 2012), Ethovision ${ }^{\circledR}$ XT (Noldus et al., 2001) and idTracker (PérezEscudero et al., 2014), allows for behavioural phenotyping. These software applications can be used in combination with a video set-up, such as the highthroughput individual T-maze that I developed (Chapter 2), and allow for extraction of a wide range of behavioural parameters from video files. Furthermore, with the high-throughput individual T-maze, the behaviour of 36 individual parasitoids, each in their own T-maze, can be recorded and tracked in a standardized high-throughput, labour-efficient and cost-effective way. I developed and used this set-up for memory retention testing in the parasitoids C. glomerata and Nasonia vitripennis (Chapters 2 and 6 ).

In the field, the development of advanced insect tracking methods with video set-ups has only just begun. Recently, video set-ups were developed for 3D tracking of individual swarming mosquitoes in the field (Manoukis et al., 2014), in-flight behaviour of mosquitoes during house entry (Spitzen et al., 2016) and in this thesis I developed a multi-camera field set-up (Chapter 4). This set-up allowed me to 
observe foraging behaviour of individual parasitoids on host and non-host infested plants under field conditions. With the video recordings from these cameras, I could measure how long it took for parasitoids to find the infested plants, how they spent their time on these plants and how they interacted with non-hosts. With this set-up, I showed that detailed individual behaviours should be measured to prevent an underestimation of the effect of unreliable associative memory on parasitoid foraging behaviour. I also showed that these individual foraging parameters are more difficult to define under field conditions, since parasitoids tend to alternate between different kinds of behaviours while they are foraging. For example, host searching behaviour may be alternated with resting, cleaning or predator avoidance behaviours. Furthermore, since the multi-camera set-up only allowed for the observation of parasitoid foraging behaviour on host and non-host infested plants, it is unknown how parasitoids spent their time elsewhere and whether the behaviour during this time was related to host foraging behaviour. These observation 'gaps' underline a need for continuous tracking of individual parasitoids in the field, requiring continuous video monitoring and/or telemetry of individual parasitoids, which is not yet feasible for small flying insects (Daniel Kissling et al., 2014).

\section{Unreliable associative memory reduces parasitoid foraging efficiency}

As described above, parasitoids can improve their foraging efficiency through associative learning (Papaj and Vet, 1990; Vet et al., 1995; Hare et al., 1997; Kruidhof et al., 2015). However, there are situations where associated HIPVs may not reliably predict host presence and lead parasitoids to plants infested with nonhosts instead of hosts (Meisner et al., 2007; de Rijk et al., 2013; Peñaflor et al., 2017). Indeed, HIPVs produced by non-host infested plants can attract females of Cotesia glomerata, also after forming associative memory with a host oviposition on this plant species (Geervliet et al., 1996; Geervliet et al., 1998b; Vosteen et al., 2019). Furthermore, associative learning of these HIPVs can increase the attractiveness of these volatiles and can subsequently increase the time parasitoids spend on and around plants infested with herbivores in which they cannot reproduce (Chapters 3 and 4). I demonstrated that persistent, unreliable associative memory, formed through 3 host ovipositions on a host plant species, had a negative effect on parasitoid foraging efficiency when tested under laboratory conditions (Chapter 3). Cotesia glomerata females with persistent unreliable memory took longer to find the 
hosts, when non-hosts were present on the plant species they had previously associated with host presence. This confirmed the validity of my first hypothesis, that unreliable associative memory can reduce a parasitoid's foraging efficiency, primarily when this memory is highly persistent. However, the effects of such unreliable memories were more nuanced in the semi-field experiment (Chapter 4). Here, C. glomerata females with persistent unreliable memory started foraging on the non-host infested plants earlier, spent more time on and around these plants, visited more non-host infested plants, visited them more often and were more prone to oviposit in the non-host Mamestra brassicae, than parasitoids with reliable memory. In contrast to my expectation, however, parasitoids with unreliable memory found the host equally fast. Since the time to find a host is commonly used as a proxy for foraging efficiency, I could not conclude that persistent unreliable memory altered C. glomerata's foraging efficiency. Nevertheless, persistent unreliable memory did alter $C$. glomerata's foraging behaviour and may still lead to negative fitness effects over the parasitoid's lifetime when they continue to waste time and eggs on non-host infested plants. Furthermore, both studies clearly demonstrated that associative memory does not always influence foraging behaviour favourably.

\section{Non-host-related extinction experiences alter a parasitoid's response to associated cues}

Given these costs of unreliable memory, I expected that parasitoids would alter their foraging behaviour after encountering plants with non-hosts. In C. glomerata, this altered behaviour, i.e. ceasing to respond to the associated HIPVs, could prevent time and eggs spent on plants infested with the non-host $M$. brassicae. In bees and fruit flies, the lack of an expected reward after encountering associated environmental cues, i.e. extinction experience, is known to cause either temporary or permanent behavioural alterations, or no behavioural alterations (Lagasse et al., 2009; Eisenhardt et al., 2013). In Chapter 5, I investigated whether and how different types of ecologically relevant extinction experiences would alter the conditioned host plant preference of Cotesia glomerata females, after they had learned to associate HIPVs with a host oviposition. In contrast to my expectation, these experiments showed that non-host-related extinction experiences did not change host searching behaviour, while a host-related extinction experience could. Cotesia glomerata did not alter its foraging behaviour after two types of non-host-related extinction experiences, i.e. 
contacting non-host traces for 15 minutes or an oviposition in this non-host after contacting host traces. Interestingly, the lack of a behavioural change after this second type of non-host extinction experience, suggests that either the short exposure to host traces and the non-host oviposition were insufficient to trigger a behavioural change, or that the non-host oviposition fulfilled the expectation of an oviposition, hence preventing the change in behaviour. A single non-host-related extinction experience might not be worth the investment in a change of behaviour (Dukas, 1999). Multiple non-host-related extinction experiences may, however, still cause behavioural changes, because a higher frequency of extinction experiences can have a stronger effect on the conditioned behaviour (Lagasse et al., 2009; Ecker, 2015). In contrast, a 15-minute extinction experience with host traces did alter the behaviour, though it was host plant species-specific. When parasitoids were conditioned on, and given a 15-minute extinction experience with, the host plant Brassica nigra, this extinction experience led to a temporary disappearance of the conditioned host plant preference. However, this preference re-appeared within 4 hours. These temporal dynamics may be beneficial when the lack of host finding accurately predicts current circumstances, but not future opportunities (Papaj et al., 1994). Together, these results indicate that a parasitoid's response to an extinction experience is influenced by its preparedness to learn these herbivore and plant cues (Smid and Vet, 2016).

\section{Parasitoids change their foraging behaviour after a non-host oviposition}

Parasitoids forage in habitats that contain a multitude of plant species, which can harbour a wide array of herbivorous insects (Vos et al., 2001; Dicke et al., 2009). Parasitoids are attracted by HIPVs of specific host plant species that may be induced by feeding of different herbivore species, including suitable and unsuitable species for parasitoid reproduction. Interactions with non-hosts can occur when parasitoids are attracted by HIPVs of non-host infested host plant species or when hosts and non-hosts feed simultaneously on the same plant (Bukovinszky et al., 2012; de Rijk et al., 2013). Both situations commonly occur in nature (Vos et al., 2001; de Rijk et al., 2013). Associative learning can improve the ability of parasitoids to discriminate between plant infested with host or non-hosts, but this is not always the case (Bukovinszky et al., 2012; de Rijk et al., 2013). Even after associative learning, $C$. glomerata is still attracted to HIPVs of non-host infested plants (Vosteen et al., 
2019), and surprisingly I found that this can also lead to a higher tendency to oviposit in the non-host $M$. brassicae caterpillars (Chapters 3 and 4). This non-host oviposition behaviour seems counter intuitive, because the eggs deposited by $C$. glomerata cannot successfully develop (Chapter 6). Non-host encounters with $M$. brassicae are thought to be a likely scenario in nature, since these non-hosts commonly feed on the same host plant species as $C$. glomerata's hosts (Popova, 1994; Vos et al., 2001; Metspalu et al., 2013; Peñaflor et al., 2017). This is why I expected, that even when $C$. glomerata females would encounter and oviposit in this non-host, they would subsequently change their behaviour, to prevent a further waste of time and eggs (Chapters 3 and 4). However, C. glomerata females with and without a $M$. brassicae oviposition experience continued to contact and oviposit in $M$. brassicae caterpillars (Chapters 3, 4 and 6). Furthermore, C. glomerata did not learn to avoid odours they encountered while ovipositing in the non-host and cooccurrence of non-host $M$. brassicae and host $P$. brassicae caterpillars could make them even more likely to oviposit in this non-host (Chapter 6). The only change in behaviour I observed, was that $C$. glomerata became more motivated to forage on an infested leaf with non-hosts after a non-host oviposition, which is most likely a general effect of a parasitoid's first oviposition. It is possible that in C. glomerata, a parasitoid with a high potential fecundity (Le Masurier, 1991), the waste of eggs does not outweigh the costs of retaining and maintaining high numbers of eggs. Acceptance of non-hosts might largely depend on the quality of alternative host species in the environment and the expectation of current and future reproductive opportunities (Godfray, 1994).

\section{The context-dependency of parasitoid foraging behaviour}

Evolutionary questions on behavioural strategies that increase foraging efficiency, must be addressed in ecological context because an animal's behaviour is directly linked to its ecology and the habitat it operates in (Gordon, 2011). When environments, and the insect and plant community within them, are spatially and temporally variable, parasitoid foraging behaviour is also expected to show spatial and temporal variation, to allow continuous adaptation to local conditions (Vet et al., 1991; Vet et al., 1995; Smid and Vet, 2016). This environmental variation makes parasitoid foraging behaviour highly context-dependent (Heimpel and Casas, 2008). This context-dependency was a reappearing topic in most of the chapters of this 
thesis and made it challenging to draw general conclusions on how parasitoid foraging behaviour is shaped by information reliability. However, I agree with Gordon et al. (2011) that by studying which aspects drive this context-dependency, we can gain insight in how foraging behaviour is shaped.

Parasitoids operate in a multitrophic system. The foraging strategy of a parasitoid depends on the herbivore and plant community, the parasitoid's internal state and the prevailing abiotic conditions (Lewis et al., 1998; Randlkofer et al., 2010). Some of these aspects have very general effects, such as the parasitoid's internal state and abiotic conditions. A parasitoid's degree of hunger and thirst, their age, mating status, egg load, disease and prior experience, are all known to affect their foraging behaviour (Prokopy and Lewis, 1993; Geervliet et al., 1998b; Lewis et al., 1998). A hungry parasitoid for example, will forage for food rather than hosts (Lewis and Takasu, 1990). Likewise, prevailing weather conditions, e.g. strong winds, rain and extreme temperatures, can limit foraging time in parasitoids (Steinberg et al., 1992; Fournier et al., 2005). The other aspects, i.e. the herbivore and plant species of the community, affect foraging behaviour in a less straightforward way, because, at the species level, they have co-evolved with each other. Parasitoid species are highly diverse (Forbes et al., 2018), since each species has its own life history and associated foraging strategy (Vet et al., 1995), which has evolved through interactions with various plant and herbivore species that naturally occur in a parasitoid's habitat. Parasitoids have evolved to use HIPVs, as a solution to find their inconspicuous herbivorous hosts, which are selected to minimise direct information transfer to their natural enemies (Vet et al., 1991). However, these volatiles vary considerably within and between plant species (Aartsma et al., 2019) and can be difficult to detect by the parasitoid, especially in environments with background vegetation consisting of non-host plants that may mask HIPVs from host plants (Randlkofer et al., 2010). Indeed, different plant species can produce considerably different HIPV blends when attacked by the same herbivore species (Bukovinszky et al., 2005; Gols et al., 2009). In contrast, HIPV blends induced by different herbivore species feeding on the same plant species may be so similar that parasitoids are unable to discriminate between host and non-host infested plants (Geervliet et al., 1996; Vos et al., 2001; Tamò et al., 2006; Randlkofer et al., 2010; Aartsma et al., 2019). Discrimination between host and non-host infested plants is especially difficult when both herbivore species are from the same feeding guild (de Rijk et al., 2013). In order to subsequently find and identify host species on these plants, 
parasitoids show a herbivore species-specific response to herbivore-related cues on the plant, e.g. faeces, silk, honeydew, and other body secretions. When non-hosts are encountered, they are generally not accepted for oviposition, since these species do not support the development of their offspring (Kraaijeveld et al., 1998). However, non-host oviposition behaviour has been observed before, both in the laboratory and the field (Takabayashi et al., 1995; Takasu and Lewis, 2003; Bukovinszky et al., 2012; Chabaane et al., 2015), and it can be stimulated through contact with host traces (Chapters 5,6) or prior host oviposition experience on the same plant species on which non-hosts are encountered (Chapters 3-6).

Studies on associative learning in insects are now starting to consider this contextdependency, by formulating a species-specific preparedness to learn (Hoedjes et al., 2011; Smid and Vet, 2016). As mentioned before, an insect's preparedness to learn consists of the ease with which a certain association is formed and how this information is stored in memory. Associative learning with different host species can for example lead to memories that differ in their persistence (Kruidhof et al., 2012). In this thesis, I show that host plant species is also an important part of a parasitoid's preparedness to learn. Plant species affected associative learning and the consequences of unreliable memory (Chapter 4) and extinction experiences (Chapter 5). When C. glomerata females foraged around Brassica nigra and Sinapis arvensis plants, the high attractiveness of B. nigra plants could overrule the effects of unreliable memory (Chapter 3 and 4). Furthermore, an associative memory was differently affected by an extinction experience, when $C$. glomerata was conditioned on either B. nigra or Brassica oleracea var. rubra plants (Chapter 5). This also shows that a parasitoid's preparedness to learn influences how it responds to subsequent experiences.

Another context-dependent aspect, is the complexity of the foraging situation. Most of what we know about parasitoid foraging behaviour is based on simplified foraging situations in laboratory studies. These simple foraging situations, however, do not represent the complexity and dynamics of natural habitats in which the behaviour has evolved. To advance our understanding of parasitoid foraging behaviour, laboratory knowledge on foraging needs to verified in the field (Heimpel and Casas, 2008). However, field testing is laborious and there are few methods to study foraging behaviour in the field (Chapter 4). Despite these challenges, the verification of foraging theories in the field has received more attention in the past decade, where 
results indeed show that parasitoid foraging behaviour is often context-dependent (Bukovinszky et al., 2012; Chabaane et al., 2015; Kruidhof et al., 2015; De Rijk et al., 2018). A combination of laboratory and field studies, however, remains ideal. Laboratory studies can inform us about the abilities of a parasitoid, while field studies show how they use these abilities in a natural environment. Laboratory testing, with methods such as the high-throughput individual T-maze (Chapter 2), allows for fast screening of abilities (e.g. associative learning) of multiple parasitoid species. More ecologically relevant laboratory set-ups can give an indication on how they can use these abilities while foraging (Chapter 3). And field studies can verify how these abilities influence parasitoid foraging behaviour, foraging efficiency (Chapter 4) and ultimately fitness, in complex habitats where this behaviour has evolved.

\section{Parasitoid learning and its implications for biological control}

Learning can improve an insect's foraging efficiency (Papaj and Vet, 1990; Hare et al., 1997; Durier and Rivault, 2000; Chittka and Muller, 2009; Kruidhof et al., 2015), which is why implementation of learning in biological control is receiving more attention lately (Giunti et al., 2015; Kruidhof et al., 2019; Little et al., 2019). It could make biological control agents, such as parasitoids, more effective in suppressing pest populations. Parasitoids need to initiate host searching behaviour, locate host infested plants and find and accept the host on this plant. During each of these stages, learning may improve the parasitoid's host foraging behaviour. Learning can enhance a parasitoid's response to HIPVs, its ability to locate host infested plants, its ability to locate the host on these plants, and its host acceptance for egg deposition (Kruidhof et al., 2019). In theory, this means that after learning to associate volatiles of the target crop with presence of the target pest (host), released parasitoids will be retained in the crop, they will more actively search for the pest, and they will be better at finding pest-infested crop plants.

Ample studies have shown these retaining and activating effects of associative learning in parasitoids, though they are often not explicitly stated, because they are not of primary interest. In wind tunnel studies, prior experience on an infested host plant can cause $30-40 \%$ increases in parasitoids that display odour guided foraging behaviour (Fukushima et al., 2001; de Bruijn et al., 2018b). Other studies have 
demonstrated that parasitoids can be conditioned to respond to HIPVs of host infested plant species that are innately not attractive (Metspalu et al., 2003; Smid et al., 2007) and that prior oviposition experience can increase host acceptance (Kerguelen and Cardé, 1996). However, in this thesis I show that persistent memory can also increase non-host acceptance when non-hosts are feeding on a plant species that was previously associated with host presence (Chapter 3 and 4). This indicates, that associative learning prior to parasitoid release might increase non-target effects. However, I expect these effects to be specific to the associated target crop, parasitoid species-specific, context-dependent and to only occur after spaced conditioning, since a single oviposition experience and contact with host traces did not make $C$. glomerata more willing to oviposit in the non-host M. brassicae (Chapter 3). Such non-target effect should be considered when implementing learning in biological control, but I expect them to be rare and unlikely to cause undesired effects.

Interestingly, even when conditioned $C$. glomerata parasitoids interacted with traces of the non-host M. brassicae or oviposited in them, they did not alter their foraging behaviour. In other words, these extinction experiences did not alter their conditioned host plant preference (Chapters 5 and 6). This indicates that associative memories could be quite resistant to extinction experiences when parasitoids forage in crops with both host and non-host herbivores in the crop. These non-host ovipositions can lead to a waste of eggs (Chapter 6), but this may not be a problem for parasitoids with high egg loads, such as C. glomerata and Aphidius colemani. Furthermore, it may still have a negative impact on the non-host due to nonreproductive effects, such as an increased mortality risk or reduced reproduction due to immune defence costs after encapsulation of eggs (Abram et al., 2019). Cotesia glomerata is not a common biological control agent, but my findings show that further research on the costs and benefits of the incorporation of parasitoid conditioning in biological control is needed. In order to improve the efficacy of biological control agents, Kruidhof et al. (2019), Giunti et al. (2015) and Little et al. (2019) all specify a relatively similar research approach. As a first step, parasitoid should be screened for their preparedness to learn to associate the HIPVs emitted by the target crop with a pest-related reward (e.g. host traces or a host oviposition). This could be done efficiently with methods such as the high-throughput individual Tmaze (Chapter 2). It may also be possible to improve learning capability through selection lines and incorporation of conditioning before parasitoid release. 


\section{Chapter 7}

Furthermore, biological control methods could be further optimized by studying parasitoids foraging behaviour in crops, in greenhouses and agricultural fields.

\section{General conclusions and future perspectives}

Natural environments consist of insect and plant communities that are often spatially and temporally variable. This has led to the development of behavioural traits, such as associative learning, that allow insects, such as parasitoids, to improve their foraging efficiency, through a temporal specialisation to local conditions. Since environments continue to change, this associative memory can become unreliable and can lead parasitoids to plants that contain non-hosts instead of hosts. In this thesis, I developed methods to study how associative learning, unreliable memory, extinction experiences and non-host interactions, shape the foraging behaviour of the parasitoid Cotesia glomerata. I developed and tested three hypotheses that were discussed in the above subsections and based on my findings, I conclude that unreliable associative memory can have a negative effect on the foraging behaviour of C. glomerata and that interactions with the non-host M. brassicae, and extinction experiences with this non-host, have little to no effect on the foraging behaviour of C. glomerata. Furthermore, throughout my thesis, the context-dependency of parasitoid foraging behaviour was an important aspect and I showed that the host plant species influences the effects of associative learning and extinction experiences. This highlights how important it is to consider a parasitoid's preparedness to learn, when testing parasitoid foraging behaviour in an ecological context, but also when considering the implementation of learning in biological control. I propose to verify and extend my research with other species of parasitoids, hosts, non-hosts and (host) plants, to better understand how these contexts influenced parasitoid foraging behaviour and the effects of unreliable memory, extinction experiences and non-host interactions.

In this thesis, I developed new methods to study parasitoid foraging behaviour in relation to associative learning. With these methods, it is possible to test various behaviours of a much wider range of parasitoid species and their use could even be extended to include a wide diversity of insect species and research fields. The highthroughput individual T-maze could be used to test an insect's response to various host, non-host and host plant-related cues, which could be highly beneficial for 
biological control practices, but also to further develop our understanding of insect foraging behaviour. Furthermore, a multi-camera system (Chapter 4) could elucidate how insects move around and interact with various components in their habitat, such as food, hosts, prey and mates. These observations could be used to test and improve various assumptions of foraging models and provide new insights on how insects spend their time foraging. For example, such an approach could reveal which rules determine insects' patch residence times and which factors (e.g. experience, nonhosts) influence this time allocation.

Overall, this thesis contributed to the general understanding of parasitoid foraging behaviour. It provides new methods to test insect foraging behaviour in the laboratory and in the field, and the first insights into the effects of unreliable memory and extinction experiences on parasitoid foraging in an ecological context, with hosts and their associated host plant species.

\section{Acknowledgments}

I thank Jetske de Boer, Hans Smid and Louise Vet for their constructive comments and advice on a previous version of this chapter. 



\section{References}



Aartsma, Y., Leroy, B., Van Der Werf, W., Dicke, M., Poelman, E.H., and Bianchi, F.J.J.A. (2019). Intraspecific variation in herbivore-induced plant volatiles influences the spatial range of plant-parasitoid interactions. Oikos 128, 7786.

Abram, P.K., Brodeur, J., Urbaneja, A., and Tena, A. (2019). Nonreproductive Effects of Insect Parasitoids on Their Hosts. 64, 259-276.

Agerbirk, N., Warwick, S.I., Hansen, P.R., and Olsen, C.E. (2008). Sinapis phylogeny and evolution of glucosinolates and specific nitrile degrading enzymes. Phytochemistry 69, 2937-2949.

Arthur, A., Hegdekar, B., and Batsch, W. (1972). A chemically defined, synthetic medium that induces oviposition in the parasite Itoplectis conquisitor (Hymenoptera: Ichneumonidae). The Canadian Entomologist 104, 12511258.

Arthur, A., Hegdekar, B., and Rollins, L. (1969). Component of the host haemolymph that induces oviposition in a parasitic insect. Nature 223, 966.

Askew, R., and Shaw, M.R. (1986). Parasitoid communities: their size, structure and development, in: Waage, $J$ and Greathead, D (eds), Insect Parasitoids, 13th Symposium of Royal Entomological Society of London: Academic Press, London (now Elsevier), 225-264.

Bates, D., Maechler, M., Bolker, B., and Walker, S. (2014). lme4: Linear mixedeffects models using Eigen and S4. R package version 1, 1-23.

Bell, D.T., and Muller, C.H. (1973). Dominance of California Annual Grasslands by Brassica nigra. The American Midland Naturalist 90, 277-299.

Benson, J., Van Driesche, R., Pasquale, A., and Elkinton, J. (2003). Introduced braconid parasitoids and range reduction of a native butterfly in New England. Biological Control 28, 197-213.

Beshel, J., and Zhong, Y. (2013). Graded encoding of food odor value in the Drosophila brain. The Journal of Neuroscience 33, 15693-15704.

Bitterman, M., Menzel, R., Fietz, A., and Schäfer, S. (1983). Classical conditioning of proboscis extension in honeybees (Apis mellifera). Journal of Comparative Psychology 97, 107.

Bleeker, M.A.K., Smid, H.M., Steidle, J.L.M., Kruidhof, H.M., Van Loon, J.J.A., and Vet, L.E.M. (2006). Differences in memory dynamics between two closely related parasitoid wasp species. Animal Behaviour 71, 1343-1350.

Brodeur, J., Geervliet, J.B.F., and Vet, L.E.M. (1996). The role of host species, age and defensive behaviour on ovipositional decisions in a solitary specialist and gregarious generalist parasitoid (Cotesia species). Entomologia Experimentalis et Applicata 81, 125-132.

Brodeur, J., and Vet, L.E.M. (1995). Relationships between parasitoid host range and host defence: a comparative study of egg encapsulation in two related parasitoid species. Physiological Entomology 20, 7-12. 
Bukovinszky, T., Gols, R., Posthumus, M.A., Vet, L.E.M., and Van Lenteren, J.C. (2005). Variation in plant volatiles and attraction of the parasitoid diadegma semiclausum (Hellen). Journal of chemical ecology 31, 461-480.

Bukovinszky, T., Poelman, E.H., Kamp, A., Hemerik, L., Prekatsakis, G., and Dicke, M. (2012). Plants under multiple herbivory: consequences for parasitoid search behaviour and foraging efficiency. Animal Behaviour 83, 501-509.

Carter, D.J. (1984). Pest Lepidoptera of Europe: with special reference to the British Isles. Springer Science \& Business Media.

Casas, J., Driessen, G., Mandon, N., Wielaard, S., Desouhant, E., Van Alphen, J., Lapchin, L., Rivero, A., Christides, J.P., and Bernstein, C. (2003). Energy dynamics in a parasitoid foraging in the wild. Journal of Animal Ecology 72, 691-697.

Celhoffer, L., Boukydis, C., Minde, K., Muir, E. (1977). The DCR-II event recorder: A portable high-speed digital cassette system with direct computer access. Behavior Research Methods \& Instruments 9, 442-446.

Chabaane, Y., Laplanche, D., Turlings, T.C.J., and Desurmont, G.A. (2015). Impact of exotic insect herbivores on native tritrophic interactions: a case study of the African cotton leafworm, Spodoptera littoralis and insects associated with the field mustard Brassica rapa. Journal of Ecology 103, 109-1 $\chi$.

Chen, C., and Tonegawa, S. (1997). Molecular genetic analysis of synaptic plasticity, activity-dependent neural development, learning, and memory in the mammalian brain. Annual review of neuroscience 20, 157-184.

Chittka, L., and Muller, H. (2009). Learning, specialization, efficiency and task allocation in social insects. Communicative \& Integrative Biology 2, 151154.

Cholé, H., Junca, P., and Sandoz, J.-C. (2015). Appetitive but not aversive olfactory conditioning modifies antennal movements in honeybees. Learning \& memory 22, 604-616.

Clavijo Mccormick, A., Unsicker, S.B., and Gershenzon, J. (2012). The specificity of herbivore-induced plant volatiles in attracting herbivore enemies. Trends in Plant Science 17, 303-310.

Coleman, R., Barker, A., and Fenner, M. (1999). Parasitism of the herbivore Pieris brassicae L.(Lep., Pieridae) by Cotesia glomerata L.(Hym., Braconidae) does not benefit the host plant by reduction of herbivory. Journal of Applied Entomology 123, 171-177.

Colomb, J., Reiter, L., Blaszkiewicz, J., Wessnitzer, J., and Brembs, B. (2012). Open Source Tracking and Analysis of Adult Drosophila Locomotion in Buridan's Paradigm with and without Visual Targets. PLOS ONE 7, e42247.

Costa, A., Ricard, I., Davison, A.C., and Turlings, T.C.J. (2010). Effects of Rewarding and Unrewarding Experiences on the Response to Host-induced 
Plant Odors of the Generalist Parasitoid Cotesia marginiventris (Hymenoptera: Braconidae). Journal of Insect Behavior 23, 303-318.

Couvillon, P., and Bitterman, M. (1980). Some phenomena of associative learning in honeybees. Journal of Comparative and Physiological Psychology 94, 878.

Dall, S.R.X., Giraldeau, L.-A., Olsson, O., Mcnamara, J.M., and Stephens, D.W. (2005). Information and its use by animals in evolutionary ecology. Trends in Ecology \& Evolution 20, 187-193.

Daniel Kissling, W., Pattemore, D.E., and Hagen, M. (2014). Challenges and prospects in the telemetry of insects. 89, 511-530.

Darling, D.C., and Werren, J.H. (1990). Biosystematics of Nasonia (Hymenoptera: Pteromalidae): Two New Species Reared from Birds' Nests in North America. Annals of the Entomological Society of America 83, 352-370.

De Bruijn, J.A.C., Vet, L.E.M., Jongsma, M.A., and Smid, H.M. (2018a). Automated high-throughput individual tracking system for insect behavior: Applications on memory retention in parasitic wasps. Journal of Neuroscience Methods 309, 208-217.

De Bruijn, J.A.C., Vet, L.E.M., and Smid, H.M. (2018b). Costs of Persisting Unreliable Memory: Reduced Foraging Efficiency for Free-Flying Parasitic Wasps in a Wind Tunnel. Frontiers in Ecology and Evolution 6.

De Jong, R., and Kaiser, L.J.J.O.I.B. (1991). Odor learning by Leptopilina boulardi, a specialist parasitoid (Hymenoptera: Eucoilidae). 4, 743-750.

De Rijk, M., Cegarra Sánchez, V., Smid, H.M., Engel, B., Vet, L.E.M., and Poelman, E.H. (2018). Associative learning of host presence in non-host environments influences parasitoid foraging. Ecological Entomology 43, 318-325.

De Rijk, M., Dicke, M., and Poelman, E.H. (2013). Foraging behaviour by parasitoids in multiherbivore communities. Animal Behaviour 85, 15171528.

De Rijk, M., Zhang, X., Van Der Loo, J.A., Engel, B., Dicke, M., and Poelman, E.H. (2016). Density-mediated indirect interactions alter host foraging behaviour of parasitoids without altering foraging efficiency. Ecological entomology 41, 562-571.

Desurmont, G.A., Guiguet, A., and Turlings, T.C. (2018). Invasive insect herbivores as disrupters of chemically-mediated tritrophic interactions: effects of herbivore density and parasitoid learning. Biological Invasions 20, 195-206.

Dicke, M., Van Loon, J.J., and Soler, R. (2009). Chemical complexity of volatiles from plants induced by multiple attack. Nature Chemical Biology 5, 317.

Dubnau, J. (2003). Neurogenetic dissection of conditioned behavior: evolution by analogy or homology? Journal of neurogenetics 17, 295-326.

Dukas, R. (1999). Costs of memory: ideas and predictions. Journal of theoretical biology 197, 41-50. 
Dunlap, A.S., and Stephens, D.W. (2016). Reliability, uncertainty, and costs in the evolution of animal learning. Current Opinion in Behavioral Sciences 12, 73-79.

Durier, V., and Rivault, C. (2000). Learning and foraging efficiency in German cockroaches, Blattella germanica (L.) (Insecta: Dictyoptera). Animal Cognition 3, 139-145.

Ecker, B. (2015). Memory reconsolidation understood and misunderstood. International Journal of Neuropsychotherapy 3, 2-46.

Eisenhardt, D. (2006). Learning and memory formation in the honeybee (Apis mellifera) and its dependency on the cAMP-protein kinase A pathway. 56, 259.

Eisenhardt, D. (2012). Extinction learning in honey bees, in Honeybee Neurobiology and Behavior, 423-438.

Eisenhardt, D., Randolf, M., and Paul, R.B. (2013). Extinction Learning and Memory Formation in the Honeybee, in Handbook of Behavioral Neuroscience, 450-457.

Exton-Mcguinness, M.T.J., Lee, J.L.C., and Reichelt, A.C. (2015). Updating memories-The role of prediction errors in memory reconsolidation. Behavioural Brain Research 278, 375-384.

Faucher, C., Forstreuter, M., Hilker, M., and De Bruyne, M. (2006). Behavioral responses of Drosophila to biogenic levels of carbon dioxide depend on lifestage, sex and olfactory context. Journal of Experimental Biology 209, 27392748.

Forbes, A.A., Bagley, R.K., Beer, M.A., Hippee, A.C., and Widmayer, H.A. (2018). Quantifying the unquantifiable: why Hymenoptera, not Coleoptera, is the most speciose animal order. BMC Ecology 18, 21.

Fournier, F., Pelletier, D., Vigneault, C., Goyette, B., and Boivin, G.J.E.E. (2005). Effect of barometric pressure on flight initiation by Trichogramma pretiosum and Trichogramma evanescens (Hymenoptera: Trichogrammatidae). 34, 1534-1540.

Fukushima, J., Kainoh, Y., Honda, H., and Takabayashi, J. (2001). Learning of hostinfested plant volatiles in the larval parasitoid Cotesia kariyai. Entomologia Experimentalis et Applicata 99, 341-346.

Galizia, G., Eisenhardt, D., and Giurfa, M. (2011). Honeybee neurobiology and behavior: a tribute to Randolf Menzel. Springer Science \& Business Media.

Geervliet, J.B.F., Ariëns, S., Dicke, M., and Vet, L.E.M. (1998a). Long-Distance Assessment of Patch Profitability through Volatile Infochemicals by the Parasitoids Cotesia glomerata and C. rubecula (Hymenoptera: Braconidae). Biological Control 11, 113-121.

Geervliet, J.B.F., Verdel, M.S.W., Snellen, H., Schaub, J., Dicke, M., and Vet, L.E.M. (2000). Coexistence and niche segregation by field populations of 
the parasitoids Cotesia glomerata and $C$. rubecula in the Netherlands: predicting field performance from laboratory data. Oecologia 124, 55-63.

Geervliet, J.B.F., Vet, L.E.M., and Dicke, M. (1994). Volatiles from damaged plants as major cues in long-range host-searching by the specialist parasitoid Cotesia rubecula. Entomologia Experimentalis et Applicata 73, 289-297.

Geervliet, J.B.F., Vet, L.E.M., and Dicke, M. (1996). Innate responses of the parasitoids Cotesia glomerata and C. rubecula (Hymenoptera: Braconidae) to volatiles from different plant-herbivore complexes. Journal of Insect Behavior 9, 525-538.

Geervliet, J.B.F., Vreugdenhil, A.I., Dicke, M., and Vet, L.E.M. (1998b). Learning to discriminate between infochemicals from different plant-host complexes by the parasitoids Cotesia glomerata and C. rubecula. Entomologia Experimentalis et Applicata 86, 241-252.

Gernat, T., Rao, V.D., Middendorf, M., Dankowicz, H., Goldenfeld, N., and Robinson, G.E. (2018). Automated monitoring of behavior reveals bursty interaction patterns and rapid spreading dynamics in honeybee social networks. Proceedings of the National Academy of Sciences 115, 14331438.

Giunti, G., Canale, A., Messing, R.H., Donati, E., Stefanini, C., Michaud, J.P., and Benelli, G. (2015). Parasitoid learning: Current knowledge and implications for biological control. Biological Control 90, 208-219.

Godfray, H.C.J. (1994). Parasitoids: behavioral and evolutionary ecology. Princeton, New Jersey: Princeton University Press.

Gols, R., Van Dam, N.M., Raaijmakers, C.E., Dicke, M., and Harvey, J.A. (2009). Are population differences in plant quality reflected in the preference and performance of two endoparasitoid wasps? Oikos 118, 733-742.

Gordon, D.M. (2011). The fusion of behavioral ecology and ecology. Behavioral Ecology 22, 225-230.

Goulson, D., and Cory, J.S. (1995). Responses of Mamestra brassicae (Lepidoptera: Noctuidae) to crowding: interactions with disease resistance, colour phase and growth. Oecologia 104, 416-423.

Gutiérrez-Ibáñez, C., Villagra, C.A., and Niemeyer, H.M. (2007). Pre-pupation behaviour of the aphid parasitoid Aphidius ervi (Haliday) and its consequences for pre-imaginal learning. Naturwissenschaften 94, 595-600.

Hare, J.D., Morgan, D.J.W., and Nguyun, T. (1997). Increased parasitization of California red scale in the field after exposing its parasitoid, Aphytis melinus, to a synthetic kairomone. 82, 73-81.

Harvey, J.A. (2000). Dynamic effects of parasitism by an endoparasitoid wasp on the development of two host species: implications for host quality and parasitoid fitness. Ecological Entomology 25, 267-278. 
Hays, D.B., and Vinson, S.B. (1971). Acceptance of Heliothis virescens (F.) (Lepidoptera, noctuidae) as a host by the parasite Cardiochiles nigriceps viereck (Hymenoptera, braconidae). Animal Behaviour 19, 344-352.

Heimpel, G.E., and Casas, J. (2008). Parasitoid foraging and oviposition behavior in the field. Behavioral ecology of insect parasitoids, 51-70.

Hilker, M., and Mcneil, J. (2008). Chemical and Behavioral Ecology in Insect Parasitoids: How to Behave Optimally in a Complex Odorous Environment, in Behavioral Ecology of Insect Parasitoids, 92-112.

Hoedjes, K.M., Kralemann, L.E.M., Van Vugt, J.J.F.A., Vet, L.E.M., and Smid, H.M. (2014). Unravelling reward value: the effect of host value on memory retention in Nasonia parasitic wasps. Animal behaviour 96, 1-7.

Hoedjes, K.M., Kruidhof, H.M., Huigens, M.E., Dicke, M., Vet, L.E.M., and Smid, H.M. (2011). Natural variation in learning rate and memory dynamics in parasitoid wasps: opportunities for converging ecology and neuroscience. Proceedings of the Royal Society B 278, 889-897.

Hoedjes, K.M., and Smid, H.M. (2014). Natural variation in long-term memory formation among Nasonia parasitic wasp species. Behavioural Processes $105,40-45$.

Hoedjes, K.M., Smid, H.M., Schijlen, E.G.W.M., Vet, L.E.M., and Van Vugt, J.J.F.A. (2015). Learning-induced gene expression in the heads of two Nasonia species that differ in long-term memory formation. BMC genomics $16,162$.

Hoedjes, K.M., Steidle, J.L., Werren, J.H., Vet, L.E.M., and Smid, H.M. (2012). High-throughput olfactory conditioning and memory retention test show variation in Nasonia parasitic wasps. Genes Brain Behavior 11, 879-887.

Huigens, M.E., Pashalidou, F.G., Qian, M.H., Bukovinszky, T., Smid, H.M., Van Loon, J.J., Dicke, M., and Fatouros, N.E. (2009). Hitch-hiking parasitic wasp learns to exploit butterfly antiaphrodisiac. Proceedings of the National Academy of Science of the USA 106, 820-825.

Ishii, Y., and Shimada, M. (2009). The effect of learning and search images on predator-prey interactions. Population Ecology 52, 27.

Jackman, S., Fearon, J., Jackman, M.S., and Mcmcpack, S. (2007). The pscl package. Soware. http://cran. rproject. org/src/contrib/Descriptions/pscl. html.

Jiang, H., Hanna, E., Gatto, C.L., Page, T.L., Bhuva, B., and Broadie, K. (2016). A fully automated Drosophila olfactory classical conditioning and testing system for behavioral learning and memory assessment. Journal of neuroscience methods 261, 62-74.

Kaiser, L., Pérez-Maluf, R., Sandoz, J.C., and Pham-Delègue, M.H. (2003). Dynamics of odour learning in Leptopilina boulardi, a hymenopterous parasitoid. Animal Behaviour 66, 1077-1084. 
Kawamata, R., Sato, Y., Suzuki, M., and Kainoh, Y. (2018). Color Preference and Associative Color Learning in a Parasitoid Wasp, Ascogaster reticulata (Hymenoptera: Braconidae). Journal of Insect Behavior 31, 523-534.

Kerguelen, V., and Cardé, R.T. (1996). Increased host acceptance in experienced females of the parasitoid Brachymeria intermedia: which types of oviposition behaviour contribute to experience? 78, 95-103.

Kloth, K.J., Ten Broeke, C.J., Thoen, M.P., Hanhart-Van Den Brink, M., Wiegers, G.L., Krips, O.E., Noldus, L.P., Dicke, M., and Jongsma, M.A. (2015). High-throughput phenotyping of plant resistance to aphids by automated video tracking. Plant methods 11, 1.

Kohn, N.R., Reaume, C.J., Moreno, C., Burns, J.G., Sokolowski, M.B., and Mery, F. (2013). Social Environment Influences Performance in a Cognitive Task in Natural Variants of the Foraging Gene. PLOS ONE 8, e81272.

Koops, M.A. (2004). Reliability and the value of information. Animal Behaviour 67, 103-111.

Kraaijeveld, A.R., Van Alphen, J.J.M., and Godfray, H.C.J. (1998). The coevolution of host resistance and parasitoid virulence. Parasitology 116, S29-S45.

Kruidhof, H.M., Kostenko, O., Smid, H.M., and Vet, L.E.M. (2019). Integrating Parasitoid Olfactory Conditioning in Augmentative Biological Control: Potential Impact, Possibilities, and Challenges. Frontiers in Ecology and Evolution 7.

Kruidhof, H.M., Pashalidou, F.G., Fatouros, N.E., Figueroa, I.A., Vet, L.E.M., Smid, H.M., and Huigens, M.E. (2012). Reward value determines memory consolidation in parasitic wasps. PLoS One 7, e39615.

Kruidhof, H.M., Roberts, A.L., Magdaraog, P., Munoz, D., Gols, R., Vet, L.E.M., Hoffmeister, T.S., and Harvey, J.A. (2015). Habitat complexity reduces parasitoid foraging efficiency, but does not prevent orientation towards learned host plant odours. Oecologia 179, 353-361.

Lagasse, F., Devaud, J.M., and Mery, F. (2009). A switch from cycloheximideresistant consolidated memory to cycloheximide-sensitive reconsolidation and extinction in Drosophila. Journal of the Neurological Sciences 29, 22252230 .

Laing, J., and Levin, D. (1982). A review of the biology and a bibliography of Apanteles glomeratus (L.)(Hymenoptera: Braconidae). Biocontrol News and Information 3, 7-23.

Le Masurier, A. (1991). Effect of host size on clutch size in Cotesia glomerata. The Journal of Animal Ecology, 107-118.

Lee, J.L. (2008). Memory reconsolidation mediates the strengthening of memories by additional learning. Nature neuroscience 11 .

Lenth, R.V. (2016). Least-squares means: the R package 1smeans. Journal of statistical software 69, 1-33. 
Lewis, W., and Martin, W. (1990). Semiochemicals for use with parasitoids: status and future. Journal of Chemical Ecology 16, 3067-3089.

Lewis, W., Stapel, J.O., Cortesero, A.M., and Takasu, K.J. (1998). Understanding how parasitoids balance food and host needs: importance to biological control. Biological control 11, 175-183.

Lewis, W., and Takasu, K. (1990). Use of learned odours by a parasitic wasp in accordance with host and food needs. Nature 348, 635-636.

Lewis, W., and Tumlinson, J.H. (1988). Host detection by chemically mediated associative learning in a parasitic wasp. Nature 331, 257.

Lewis, W.J., Jones, R.L., Nordlund, D.A., and Gross, H.R. (1975). Kairomones and their use for management of entomophagous insects: II. Mechanisms causing increase in rate of parasitization by Trichogramma spp. Journal of Chemical Ecology 1, 349-360.

Liefting, M., Hoedjes, K.M., Lann, C.L., Smid, H.M., and Ellers, J. (2018). Selection for associative learning of colour stimuli reveals correlated evolution of this learning ability across multiple stimuli and rewards. Evolution 72, 14491459.

Lin, C.-C., Prokop-Prigge, K.A., Preti, G., and Potter, C.J. (2015). Food odors trigger Drosophila males to deposit a pheromone that guides aggregation and female oviposition decisions. Elife 4, e08688.

Little, C.M., Chapman, T.W., and Hillier, N.K. (2019). Considerations for Insect Learning in Integrated Pest Management. Journal of Insect Science (Online) $19,6$.

Lucas-Barbosa, D., Poelman, E.H., Aartsma, Y., Snoeren, T.A., Van Loon, J.J., and Dicke, M. (2014). Caught between parasitoids and predators-survival of a specialist herbivore on leaves and flowers of mustard plants. Journal of chemical ecology 40, 621-631.

Luo, S., Michaud, J.P., Li, J., Liu, X., and Zhang, Q. (2013). Odor learning in Microplitis mediator (Hymenoptera: Braconidae) is mediated by sugar type and physiological state. Biological Control 65, 207-211.

Manoukis, N.C., Butail, S., Diallo, M., Ribeiro, J.M.C., and Paley, D.A. (2014). Stereoscopic video analysis of Anopheles gambiae behavior in the field: challenges and opportunities. Acta tropica 132, S80-S85.

Manoukis, N.C., and Collier, T.C. (2019). Computer Vision to Enhance Behavioral Research on Insects. Annals of the Entomological Society of America 112, 227-235.

Margulies, C., Tully, T., and Dubnau, J. (2005). Deconstructing memory in Drosophila. Current Biology 15, R700-R713.

Mcauslane, H.J., Vinson, S.B., and Williams, H.J. (1991). Stimuli influencing host microhabitat location in the parasitoid Campoletis sonorensis. Entomologia Experimentalis et Applicata 58, 267-277. 
Meisner, M., Harmon, J.P., and Ives, A.R. (2007). Presence of an unsuitable host diminishes the competitive superiority of an insect parasitoid: a distraction effect. Population Ecology 49, 347-355.

Menzel, R. (1968). Das Gedächtnis der Honigbiene für Spektralfarben. Zeitschrift für vergleichende Physiologie 60, 82-102.

Menzel, R. (1999). Memory dynamics in the honeybee. Journal of Comparative Physiology A 185, 323-340.

Mery, F., and Kawecki, T.J. (2004). An operating cost of learning in Drosophila melanogaster. Animal Behaviour 68, 589-598.

Metspalu, L., Hiiesaar, K., Joudu, J., and Kuusik, A. (2003). Influence of food on the growth, development and hibernation of large white butterfly (Pieris brassicae). Agronomy Research 1, 85-92.

Metspalu, L., Kruus, E., Jogar, K., Kuusik, A., Williams, I.H., Veromann, E., Luik, A., Ploomi, A., Hiiesaar, K., and Kivimaegi, I. (2013). Larval food plants can regulate the cabbage moth, Mamestra brassicae population. Bulletin of insectology 66, 93-101.

Noldus, L.P., Spink, A.J., and Tegelenbosch, R.A. (2001). EthoVision: a versatile video tracking system for automation of behavioral experiments. Behavior Research Methods, Instruments, \& Computers 33, 398-414.

Papaj, D.R., and Prokopy, R.J. (1989). Ecological and evolutionary aspects of learning in phytophagous insects. Annual review of entomology 34, 315-350.

Papaj, D.R., Snellen, H., Swaans, K., and Vet, L.E.M. (1994). Unrewarding experiences and their effect on foraging in the parasitic wasp Leptopilina heterotoma (Hymenoptera: Eucoilidae). Journal of insect behavior 7, 465481.

Papaj, D.R., and Vet, L.E.M. (1990). Odor learning and foraging success in the parasitoid, Leptopilina heterotoma. Journal of Chemical Ecology 16, 31373150 .

Peñaflor, M.F.G., Gonçalves, F.G., Colepicolo, C., Sanches, P.A., and Bento, J.M.S. (2017). Effects of single and multiple herbivory by host and non-host caterpillars on the attractiveness of herbivore-induced volatiles of sugarcane to the generalist parasitoid Cotesia flavipes. Entomologia Experimentalis et Applicata 165, 83-93.

Pérez-Escudero, A., Vicente-Page, J., Hinz, R.C., Arganda, S., and De Polavieja, G.G. (2014). idTracker: tracking individuals in a group by automatic identification of unmarked animals. Nature Methods 11, 743.

Perfecto, I., and Vet, L.E.M. (2003). Effect of a Nonhost Plant on the Location Behavior of Two Parasitoids: The Tritrophic System of Cotesia spp. (Hymenoptera: Braconidae), Pieris rapae (Lepidoptera: Pieridae), and Brassica oleraceae. Environmental Entomology 32, 163-174. 
Pinheiro, J., Bates, D., Debroy, S., and Sarkar, D. (2014). R Core Team (2014) nlme: linear and nonlinear mixed effects models. R package version 3.1-117. Available at $h \mathrm{ttp}: / / C R A N$. $R$-project. org/package $=n l m e$.

Poelman, E.H., Oduor, A.M.O., Broekgaarden, C., Hordijk, C.A., Jansen, J.J., Van Loon, J.J.A., Van Dam, N.M., Vet, L.E.M., and Dicke, M. (2009). Field parasitism rates of caterpillars on Brassica oleracea plants are reliably predicted by differential attraction of Cotesia parasitoids. Functional Ecology 23, 951-962.

Popova, T. (1994). A study of antibiotic effect of cabbage cultivars on the cabbage moth Mamestra brassicae (L.)(Lepidoptera: Noctuidae). Entomological Review 73, 275-282.

Price, P.W. (1980). Evolutionary biology of parasites. Princeton University Press.

Prokopy, R.J., and Lewis, W.J. (1993). Application of learning to pest management, in Insect Learning, 308-342.

Raine, N.E., and Chittka, L. (2008). The correlation of learning speed and natural foraging success in bumble-bees. Proceedings of the Royal Society B 275, 803-808.

Raine, N.E., Ings, T.C., Dornhaus, A., Saleh, N., and Chittka, L. (2006). Adaptation, Genetic Drift, Pleiotropy, and History in the Evolution of Bee Foraging Behavior, in Advances in the Study of Behavior 305-354.

Randlkofer, B., Obermaier, E., Hilker, M., and Meiners, T. (2010). Vegetation complexity-The influence of plant species diversity and plant structures on plant chemical complexity and arthropods. Basic and Applied Ecology 11, 383-395.

Reza, M.A., Mhatre, S.D., Morrison, J.C., Utreja, S., Saunders, A.J., Breen, D.E., and Marenda, D.R. (2013). Automated analysis of courtship suppression learning and memory in Drosophila melanogaster. Fly 7, 105-111.

Rijk, M.D., Sánchez, V.C., Smid, H.M., Engel, B., Vet, L.E.M., and Poelman, E.H. (2018). Associative learning of host presence in non-host environments influences parasitoid foraging. Ecological Entomology 43, 318-325.

Russell, A.L., Golden, R.E., Leonard, A.S., and Papaj, D.R. (2015). Bees learn preferences for plant species that offer only pollen as a reward. Behavioral Ecology 27, 731-740.

Salazar-Jaramillo, L., Paspati, A., Van De Zande, L., Vermeulen, C.J., Schwander, T., and Wertheim, B. (2014). Evolution of a Cellular Immune Response in Drosophila: A Phenotypic and Genomic Comparative Analysis. Genome Biology and Evolution 6, 273-289.

Signorell, A. (2016). DescTools: Tools for descriptive statistics. $R$ package version 0.9917. 
Smallegange, R.C., Van Loon, J.J.A., Blatt, S.E., Harvey, J.A., and Dicke, M. (2008). Parasitoid load affects plant fitness in a tritrophic system. Entomologia Experimentalis Et Applicata 128, 172-183.

Smid, H.M., and Vet, L.E.M. (2016). The complexity of learning, memory and neural processes in an evolutionary ecological context. Current Opinion in Insect Science 15, 61-69.

Smid, H.M., Wang, G., Bukovinszky, T., Steidle, J.L., Bleeker, M.A., Van Loon, J.J., and Vet, L.E.M. (2007). Species-specific acquisition and consolidation of long-term memory in parasitic wasps. Proceedings of the Royal Society B 274, 1539-1546.

Smith, B.H., and Burden, C.M. (2014). A proboscis extension response protocol for investigating behavioral plasticity in insects: application to basic, biomedical, and agricultural research. Journal of visualized experiments: JoVE 91, e51057.

Smith, K.E., and Raine, N.E. (2014). A comparison of visual and olfactory learning performance in the bumblebee Bombus terrestris. Behavioral Ecology and Sociobiology 68, 1549-1559.

Spitzen, J., Koelewijn, T., Mukabana, W.R., and Takken, W. (2016). Visualization of house-entry behaviour of malaria mosquitoes. Malaria Journal 15, 233.

Spitzen, J., W Spoor, C., Grieco, F., Ter Braak, C., Beeuwkes, J., P Van Brugge, S., Kranenbarg, S., Noldus, L., Van Leeuwen, J.L., and Takken, W. (2013). A 3D analysis of flight behavior of Anopheles gambiae sensu stricto Malaria Mosquitoes in Response to Human Odor and Heat. PloS one 8, e62995.

Steinberg, S., Dicke, M., Vet, L., and Wanningen, R. (1992). Response of the braconid parasitoid Cotesia $(=$ Apanteles $)$ glomerata to volatile infochemicals: effects of bioassay set-up, parasitoid age and experience and barometric flux. Entomologia experimentalis et applicata 63, 163-175.

Stephens, D.W. (1993). Learning and behavioral ecology: incomplete information and environmental predictability, in Insect learning. 195-218.

Stephens, D.W., and Krebs, J.R. (1986). Foraging theory. Princeton University Press.

Stollhoff, N., Menzel, R., and Eisenhardt, D. (2005). Spontaneous recovery from extinction depends on the reconsolidation of the acquisition memory in an appetitive learning paradigm in the honeybee (Apis mellifera). Journal of Neurological Sciences 25, 4485-4492.

Strand, M.R., and Obrycki, J.J. (1996). Host specificity of insect parasitoids and predators. BioScience 46, 422-429.

Strand, M.R., and Pech, L.L. (1995). Immunological Basis for Compatibility in Parasitoid-Host Relationships. Annual Review of Entomology 40, 31-56. 
Takabayashi, J., and Takahashi, S. (1990). An allelochemical elicits arrestment in Apanteles kariyai in feces of nonhost larvae Acantholeucania loreyi. Journal of Chemical Ecology 16, 2009-2017.

Takabayashi, J., Takahashi, S., Dicke, M., and Posthumus, M.A. (1995). Developmental stage of herbivore Pseudaletia separata affects production of herbivore-induced synomone by corn plants. Journal of Chemical Ecology 21, 273-287.

Takasu, K., and Lewis, W.J. (2003). Learning of host searching cues by the larval parasitoid Microplitis croceipes. Entomologia Experimentalis et Applicata 108, 77-86.

Takemoto, H., Kainoh, Y., and Takabayashi, J. (2011). Learning of plant volatiles by aphid parasitoids: timing to learn. Journal of Plant Interactions 6, 137140.

Tamò, C., Ricard, I., Held, M., Davison, A.C., and Turlings, T.C. (2006). A comparison of naive and conditioned responses of three generalist endoparasitoids of lepidopteran larvae to host-induced plant odours. Animal Biology 56, 205-220.

Tertuliano, M., Olson, D.M., Rains, G.C., and Lewis, W.J. (2004). Influence of handling and conditioning protocol on learning and memory of Microplitis croceipes. Entomologia Experimentalis et Applicata 110, 165-172.

Therneau, T.M., and Lumley, T. (2015). Package 'survival'. R Top Doc 128.

Thiel, A., and Hoffmeister, T.S. (2009). Decision-Making Dynamics in Parasitoids of Drosophila. Advances in parasitology 70, 45-66.

Thoen, M.P. (2016). Host-plant resistance to western flower thrips in Arabidopsis. Wageningen University.

Thoen, M.P., Kloth, K.J., Wiegers, G.L., Krips, O.E., Noldus, L.P., Dicke, M., and Jongsma, M.A. (2016). Automated video tracking of thrips behavior to assess host-plant resistance in multiple parallel two-choice setups. Plant methods $12,1$.

Tully, T., Preat, T., Boynton, S., and Del Vecchio, M. (1994). Genetic dissection of consolidated memory in Drosophila. Cell 79, 35-47.

Turlings, T.C.L., Wäckers, F.L., Vet, L.E.M., Lewis, W.J., and Tumlinson, J.H. (1993). Learning of host-finding cues by hymenopterous parasitoids, in Insect learning, 51-78.

Van Alphen, J.J., and Bernstein, C. (2008). Information acquisition, information processing, and patch time allocation in insect parasitoids, in Behavioral ecology of insect parasitoids: From Theoretical Approaches to Field Applications, 172-192.

Van Alphen, J.J., Bernstein, C., and Driessen, G. (2003). Information acquisition and time allocation in insect parasitoids. Trends in Ecology \& Evolution 18, 8187. 
Van Baalen, M., and Hemerik, L. (2008). Parasitoid fitness: from a simple idea to an intricate concept, in Behavioral Ecology of Insect Parasitoids: From Theoretical Approaches to Field Applications, 31-50.

Van Den Berg, M., Duivenvoorde, L., Wang, G., Tribuhl, S., Bukovinszky, T., Vet, L.E.M., Dicke, M., and Smid, H.M. (2011). Natural variation in learning and memory dynamics studied by artificial selection on learning rate in parasitic wasps. Animal Behaviour 81, 325-333.

Van Der Woude, E., Huigens, M.E., and Smid, H.M. (2018). Differential effects of brain size on memory performance in parasitic wasps. Animal Behaviour 141, 57-66.

Van Vugt, J.J.F.A., Hoedjes, K.M., Van De Geest, H.C., Schijlen, E.W.G.M., Vet, L.E.M., and Smid, H.M. (2015). Differentially expressed genes linked to natural variation in long-term memory formation in Cotesia parasitic wasps. Frontiers in Behavioral Neuroscience 9.

Vet, L.E.M. (2001). Parasitoid searching efficiency links behaviour to population processes.Applied Entomology and Zoology 36, 399-408.

Vet, L.E.M., and Dicke, M. (1992). Ecology of infochemical use by natural enemies in a tritrophic context. Annual review of entomology 37, 141-172.

Vet, L.E.M., Lenteren, J.C.V., Heymans, M., and Meelis, E. (1983). An airflow olfactometer for measuring olfactory responses of hymenopterous parasitoids and other small insects. Physiological Entomology 8, 97-106.

Vet, L.E.M., Lewis, W.J., and Carde, R.T. (1995). Parasitoid foraging and learning, in Chemical ecology of insects 2, 65-101.

Vet, L.E.M., Lewis, W.J., Papaj, D.R., and Van Lenteren, J.C. (1990). A variableresponse model for parasitoid foraging behavior. Journal of Insect Behavior 3, 471-490.

Vet, L.E.M., and Papaj, D.R. (1992). Effects of experience on parasitoid movement in odour plumes. Physiological Entomology 17, 90-96.

Vet, L.E.M., Wäckers, F.L., and Dicke, M. (1991). How To Hunt for Hiding Hosts: the Reliability-Detectability Problem in Foraging Parasitoids. Netherlands Journal of Zoology 41, 202-213.

Vinson, S. (1975). Biochemical coevolution between parasitoids and their hosts, in Evolutionary strategies of parasitic insects and mites, 14-48.

Vinson, S.B. (1976). Host selection by insect parasitoids. Annual review of entomology 21, 109-133.

Vinson, S.B. (1998). The General Host Selection Behavior of Parasitoid Hymenoptera and a Comparison of Initial Strategies Utilized by Larvaphagous and Oophagous Species. Biological Control 11, 79-96.

Vos, M., Berrocal, S.M., Karamaouna, F., Hemerik, L., and Vet, L. (2001). Plantmediated indirect effects and the persistence of parasitoid-herbivore communities. Ecology Letters 4, 38-45. 
Vos, M., Hemerik, L., and Vet, L.E.M. (1998). Patch exploitation by the parasitoids Cotesia rubecula and Cotesia glomerata in multi-patch environments with different host distributions. Journal of Animal Ecology 67, 774-783.

Vosteen, I., Van Den Meiracker, N., and Poelman, E.H. (2019). Getting confused: learning reduces parasitoid foraging efficiency in some environments with non-host-infested plants. Oecologia 189, 919-930.

Wäckers, F. (1994). The effect of food deprivation on the innate visual and olfactory preferences in the parasitoid Cotesia rubecula. Journal of Insect Physiology 40, 641-649.

Wäckers, F., Bonifay, C., Vet, L., and Lewis, J. (2006). Gustatory response and appetitive learning in Microplitis croceipes in relation to sugar type and concentration. Animal Biology 56, 193-203.

Wajnberg, É. (2006). Time allocation strategies in insect parasitoids: from ultimate predictions to proximate behavioral mechanisms. Behavioral Ecology and Sociobiology 60, 589-611.

Wajnberg, E., Roitberg, B.D., and Boivin, G. (2016). Using optimality models to improve the efficacy of parasitoids in biological control programmes. Entomologia Experimentalis et Applicata 158, 2-16.

Zhou, Z., Kulasiri, D., Samarasinghe, S., Rains, G., and Olson, D.M. (2015). Computational modeling and experimental validation of odor detection behaviors of classically conditioned parasitic wasp, Microplitis croceipes. Biotechnology progress 31, 596-606.

Zimmerman, P.H., Bolhuis, J.E., Willemsen, A., Meyer, E.S., and Noldus, L.P. (2009). The Observer XT: A tool for the integration and synchronization of multimodal signals. Behavior research methods 41, 731-735.

Zrelec, V., Zini, M., Guarino, S., Mermoud, J., Oppliger, J., Valtat, A., Zeender, V., and Kawecki, T.J. (2013). Drosophila rely on learning while foraging under semi-natural conditions. Ecology and evolution 3, 4139-4148. 


Summary 

Natural environments consist of complex plant and insect communities that are spatially and temporally dynamic. Foraging for resources under such conditions requires complex decision-making and has led to the evolution of behavioural traits that allow insects to use information on local conditions and adjust their behaviour accordingly. These behavioural adjustments can be achieved for example through associative learning, where an encounter with a resource (e.g. food or host) is associated with nearby environmental cues (e.g. volatiles). The resulting associative memory can improve the insect's capacity to locate these resources, i.e. improve its foraging efficiency, which is expected to ultimately increase fitness. The link between foraging behaviour and fitness is particularly strong in parasitoid wasps, because finding a suitable host is required for the production of offspring.

Parasitoids deposit their eggs in or on other organisms (generally insects), where successful offspring development results in the death of the host. Since this intimate relationship is lethal for the host, they have evolved mechanisms to kill parasitoid eggs and have become inconspicuous. Host-derived cues (e.g. frass) thus have a limited detection range, and to find their hosts parasitoids therefore primarily rely on indirect long-range cues, such as herbivore-induced plant volatiles (HIPVs). Parasitoids are expected to be genetically adapted to respond to such plant cues when they reliably predict host presence over many generations. However, due to temporal and spatial variation of host presence in the current environment, this information might not always be sufficient to successfully find hosts. In these situations, parasitoids may improve their host foraging efficiency, by associating plant speciesspecific cues (e.g. HIPVs) with a host encounter, thereby forming associative memory. This allows parasitoids to learn about the local availability and distribution of host species on particular host plant species. Nevertheless, these associative memories do not always reliably predict host presence and could lead parasitoids to plants where hosts have left the plant or where non-host instead of host herbivores are present. Such unrewarding experiences indicate that previously obtained information has become unreliable and this may lead to behavioural changes to maintain foraging efficiency.

In this thesis, I studied how unreliable associative memory, and the interactions with non-hosts, shaped the foraging behaviour of parasitoids. I developed advanced observation methods to study parasitoid foraging behaviour and used them to test the following hypotheses: 1) Unreliable associative memory reduces parasitoid foraging 
efficiency, 2) Non-host-related extinction experiences alter a parasitoid's response to associated cues, 3) Parasitoids change their foraging behaviour after a non-host oviposition. As a study system, I used the parasitoid species Cotesia glomerata (Hymenoptera: Braconidae) and Nasonia vitripennis (Hymenoptera: Pteromalidae), C. glomerata's host Pieris brassicae (Lepidoptera: Pieridae), the non-host Mamestra brassicae (Lepidoptera: Noctuidae), N. vitripennis's host Calliphora vomitoria (Diptera: Calliphoridae), and various brassicaceous host plant species.

Parasitoid foraging behaviour is complex and measuring this trait for quantitative analysis is challenging and often labour-intensive. In Chapter 2, I developed the high-throughput individual T-maze, an automated insect tracking system, with which I tested for memory retention in C. glomerata and $N$. vitripennis after associative learning with artificial odours and a host reward. With this system, I recorded and subsequently tracked the movement and choice behaviour of 36 individual parasitoids simultaneously. These experiments showed that various behavioural parameters can be used to demonstrate memory retention in both parasitoid species.

While such studies inform us on the abilities of parasitoids to learn, they are not representative for parasitoid foraging behaviour in nature. A parasitoid's foraging behaviour is linked with its life history traits, its ecology and the habitat it operates in. Foraging behaviour has evolved in this ecological context and should therefore be studied under representative, preferably field, conditions. In subsequent chapters, I incorporated this ecological context by conditioning C. glomerata on host-infested brassicaceous plants. In Chapter 3, I tested whether memories, containing unreliable information, affected the foraging efficiency for hosts in Cotesia glomerata (hypothesis 1). Parasitoids were given different kinds of learning experiences, after which they were allowed to forage in a wind tunnel set-up with host and non-host infested plants. Here, either the host or non-host infested plant species corresponded with the associated host plant species and therefore parasitoids had either reliable or unreliable memory. Since $C$. glomerata does not distinguish between HIPVs induced by $P$. brassicae caterpillars (host) and $M$. brassicae caterpillars (non-host), parasitoids were also attracted to the non-host infested plants. In this situation, parasitoids with highly persistent unreliable memory, formed by 3 ovipositions spaced in time, foraged very differently than those with persistent reliable memory. Parasitoids with persistent unreliable memory showed a high tendency to oviposit in 
non-hosts, spent more time on non-host infested plants and subsequently took longer to find the host-infested plant. These results thus supported my first hypothesis, that unreliable memory can have negative effect on parasitoid foraging efficiency. In Chapter 4, I extended verification of this hypothesis to the field, where parasitoids with persistent reliable and unreliable memory foraged up to 5 hours in a $12 \times 12$ meter tent with non-host infested plants and a host-infested plant. I developed a multi-camera system that allowed me to trace in detail how these parasitoids spent their time on and around these plants. This approach showed that the effects of persistent unreliable memory were more nuanced under field conditions than in the laboratory. The effects were host plant species-specific and although parasitoids with unreliable memory spent more time on the non-host infested plants, they did not take longer to find the host. Parasitoids with unreliable memory were, however, more likely to oviposit in the non-host, irrespective of the host plant species. Unreliable memory thus clearly resulted in a waste of time and eggs, but it remains difficult to draw a firm conclusion on its effect on $C$. glomerata's foraging efficiency.

In bees and fruit flies, the lack of an expected reward after encountering associated environmental cues, i.e. extinction experience, is known to cause either temporary or permanent behavioural alterations, or no behavioural alterations. These effects have been studied extensively in bees and fruit flies, but primarily with synthetic cues and artificial rewards. Because such cues lack natural variation and are not meaningful in an ecological context, it is questionable whether insect foraging behaviour is affected in the same way when natural environmental cues and rewards are used. In Chapter 5, I therefore tested whether and how C. glomerata altered its foraging behaviour after extinction experiences with host traces, non-host traces and host traces in combination with a non-host oviposition. Parasitoids were first given a single oviposition experience on a host plant to form an associative memory, and were then given one of these three extinction experiences. The conditioned host plant preference did not disappear after the non-host-related extinction experiences, thus not supporting my second hypothesis. However, an extinction experience with host traces did result in the disappearance of the conditioned host plant preference. The conditioned host plant preference recovered spontaneously within 4 hours after an extinction experience with host traces. This may be explained by the high potential fitness benefit of an associative memory with a host oviposition, and the fact that females were given only a single extinction experience. Interestingly, by conditioning parasitoids on two different, yet closely related, host plant species, I 
discovered that the effects of an extinction experience with host traces was host plant species-specific. Overall, these results indicate that a parasitoid's response to an extinction experience is adaptive in the context of its specific ecology and its evolutionary history with host and host plant-related cues.

In a natural environment, it is common that multiple herbivore species feed simultaneously on the same plant species, plant individual and even on the same leaf. For a parasitoid, this means that non-host encounters will be common because individual plants may be shared by hosts and non-hosts. Parasitoids are expected to ignore non-hosts, but there are various reports on oviposition in non-hosts. In previous chapters, I frequently observed that $C$. glomerata attacked and potentially deposited eggs in the caterpillars of the presumed non-host $M$. brassicae, even under field conditions. In Chapter 6, I confirmed that C. glomerata deposited eggs in $M$. brassicae, but that the caterpillar had the ability to kill the parasitoid eggs. Although egg deposition in a non-host may be maladaptive behaviour, it may allow parasitoids to gain information on their current environment, resulting in changes in subsequent foraging behaviour. This theory, i.e. my third hypothesis, was tested in Chapter 5. I showed that $C$. glomerata did not alter its foraging behaviour after a non-host oviposition in M. brassicae. In other words, females did not learn to avoid odours they encountered during the non-host oviposition and they continued to oviposit in non-host caterpillars. Furthermore, the presence of hosts stimulated C. glomerata to oviposit in non-hosts. The cost of egg deposition in non-hosts might depend on the parasitoid's egg load, the quality of alternative host species in the environment and a female's expectation of her current and future reproductive opportunities. Because C. glomerata has a high egg load, the cost of non-host acceptance may be relatively low, especially when few future reproductive opportunities are expected.

This thesis contributed to our understanding of parasitoid foraging behaviour and how it is shaped by associative learning, information-reliability, extinction experiences and interactions with non-hosts. In Chapter 7, the general discussion, I present and overview on measuring parasitoid foraging behaviour in the laboratory and the field, whether my findings support the three hypotheses I developed, the context-dependency of my results and how parasitoid learning can contribute to biological control of pest insects. Measuring parasitoid foraging behaviour is complex and labour-intensive, especially in the field. The development of the highthroughput individual T-maze and the multi-camera field set-up provided new 
opportunities to observe and measure detailed behaviours of individual parasitoids in a time-efficient manner. With these set-ups, I addressed my hypotheses experimentally and conclude that unreliable memory can indeed have a negative effect on parasitoid foraging behaviour, but that non-host interactions and non-hostrelated extinction experiences do not necessarily lead to a change in parasitoid foraging behaviour. Furthermore, my thesis showed that parasitoid foraging behaviour can be host plant species-specific and that it is thus very important to realize that parasitoid foraging behaviour is context-dependent. I discussed this context-dependency and highlighted the need for the validation of my hypotheses with other species of parasitoids, hosts, non-hosts and (host) plants. Furthermore, I discussed the implementation of parasitoid learning in biological control to enhance its efficiency. Increased non-host acceptance behaviour after associative learning suggests that associative learning may actually lead to potential non-target effects. However, based on my findings, these effects are likely to be specific to the associated target crop, parasitoid species-specific, context-dependent and only to occur after spaced conditioning. By gaining more insight on how associative learning, information reliability, extinction experiences and non-host interactions shape parasitoid foraging behaviour, our current understanding of parasitoid foraging behaviour and foraging models can be improved, which in turn can lead to the development of more effective biological control methods. 



\section{Acknowledgements}

My $\mathrm{PhD}$ was a great experience, it provided me with the freedom to design, plan and manage my own research and I loved working with parasitoids. This 4-year journey was not without hardship. Completing my thesis proved to be more of a challenge than I had anticipated and the last mile proved to be the longest. However, I managed, or I would rather say, we managed! In this section I would like to thank the people who have supported me during my $\mathrm{PhD}$, without them I would not have come this far.

Hans, I greatly appreciate that you provided me with the opportunity to work on this exciting project. I always appreciated and benefited from your supervision. You gave me a lot of freedom to develop my own research and whenever I got stuck on something I could always count on your support. My manuscripts were always full with your critical and constructive comments, which were of great help to improve their quality, and you created many beautiful illustrations for my thesis. Not only did you teach me a lot about conducting science, you also taught me how to remain sane despite the never-ending pile of work and how to supervise students. You always emphasised that despite the hard work, it was also very important to relax and have a little fun. I think we definitely achieved just that while we were doing the field experiment, hard work, yet also having fun by lazing in the sun, protecting our parasitoids against big beautiful spiders and spotting bunnies in our tents. One of the things I am most proud of is the behavioural set-ups that we built together. These set-ups will be very valuable for further research and I am secretly hoping that we will once more find an opportunity to develop and build more things together.

Jetske, without you I would not have been able to develop this thesis. In the last few months of writing my thesis, you worked through my manuscripts at an amazing speed and taught me many things about how to write an article. Though our collaboration for my $\mathrm{PhD}$ has ended, you have now become my desk neighbour at the NIOO and I hope to learn many more new things from you.

Louise, you are the most amazing woman I have ever met. Both in science and society you play a very important role, yet you always managed to make some time to see how I was doing. During our meetings you always had 10 new ideas and your brimming enthusiasm was highly contagious. One day, when I just left from Ento to go home by bike, you called me and asked me if I could come and visit you at the NIOO, since you had something to discuss with me. You seemed quite serious on 


\section{Acknowledgements}

the phone and I feared bad news. As I nervously sat at your table you told me that Olga was going to quit her postdoc project and that you would like me to succeed her. This was a complete surprize to me, yet I did not need to think twice about it and said yes with a huge smile on my face. Thank you for this opportunity. You were, and continue to be, a great source of inspiration for me, both in terms of science and lifestyle.

Olga, thank you very much for your statistical advice and for suggesting me as your successor of the boost project at the NIOO. Picking up this new project, while still working on my $\mathrm{PhD}$, was challenging, but I was very happy that we had some time to work together for a smooth transfer of the project. I will do my best to make the most out of it and I hope you will have a good time at your new job at Koppert.

Ilka, I really enjoyed working with you in the field. The many hours of watching Cotesia tv on the monitoring screen of our field experiment have payed off, as it has resulted in an amazing chapter in this thesis.

Then, a special thanks to all the people at the Laboratory of Entomology. I had a lot of fun while working there and I enjoyed the welcoming atmosphere, it really felt like I was part of the Ento-family. Hellen, Karen and Peter, I loved talking with you about managing a vegetable garden and Tessa, our talks about cooking always inspired me to try out many more new things. Antonio, it was always fun talking to you, we had many conversations about Italian food and how we Dutch people were ruining it by putting pineapple on pizza. I also had a lot of fun as a member of the Dutch speaking lunch and the party committee. Keiko, Julia, Ilka, Thibault, Max, Sandeep, Filippo, Yidong, I hope I was able to help you a bit with learning Dutch. I enjoyed teaching you together with Yavanna, Peter and Marieke and I learned a lot about the Dutch language through all of you. Being a part of the party committee was also very enjoyable. Shuhang, Julia, Sandeep, Stijn, Yavanna, Jeroen, Jitte, Max, Maite and Yidong, I think we have developed and executed many fun (and crazy) ideas for social events within our department. Especially the Sinterklaas celebration, with the dice game, creation of the poem and Joop as Sinterklaas himself, was great fun and I hope this will remain a yearly event. Furthermore, I would like to thank the insect rearing team, Léon, Pieter, Kimmy, Frans and André for rearing my insects and the Unifarm staff for rearing my plants.

I would also like to thank my family, my parents and my brother and sister. Papa en mamma, bedankt dat jullie mij de mogelijkheid hebben gegeven om te studeren wat 
$i k$ wilde. Ik heb mijn liefde voor reizen en de natuur van jullie overgenomen tijdens de vele vakanties met de caravan en camper en dit heeft een grote rol gespeeld in mijn keuze om biologie te gaan studeren. Lianne, ik heb altijd veel van jou geleerd als oudere zus en ik vond het erg leuk om te zien dat je biologische bestrijders los ging laten op de kamerplanten. Erik, met jou is er altijd lol te beleven en verder was het erg leuk om voor jou op een entomologische zoektocht te gaan om uit te zoeken wat voor kevertje er in het hout zat van jouw bedrijf.

Wouter, voor mij ben jij een geweldige levenspartner. Jouw steun, optimisme en geduld heeft mij enorm geholpen tijdens mijn $\mathrm{PhD}$. Je wist altijd mijn problemen te relativeren, zo erg was het allemaal niet. Daarnaast, in de laatste maanden van het schrijven van mijn thesis, toen de druk steeds verder opliep, was het geweldig dat je mij meenam om allerlei leuken dingen te doen om de stress een beetje te onderdrukken. You really helped me to remain sane. 



\section{About the author}

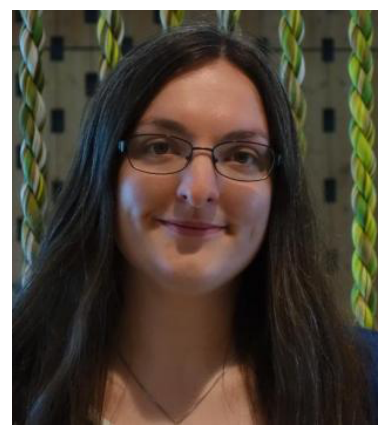

Johanna Adriana Caroline (Jessica) de Bruijn was born on 29 September 1988 in Rotterdam, the Netherlands, and grew up in the small town Westmaas. After graduating from high school in 2008, she started studying Biology at the Wageningen University. During her MSc she developed a facination for parasitoids with their diverse ecology and behaviour, which led to a MSc thesis on associative learning in the parasitoid Trichogramma evanescense at the Laboratory of Entomology. Due to her interest in biological control, she subsequently did an internship at the Commonwealth Scientific and Industrial Research Organisation (CSIRO) in Brisbane, Australia. There she studied the behaviour of the fruitspotting bug (Amblypelta nitida) for the development of a better monitoring trap. During both her MSc thesis and intership she designed and developed behavioural set-ups to study insect behaviour.

After obtaining her MSc in Biology she organised and presented various lectures on the ecology of Dutch insects for fieldbiologists, in association with the Koninklijke Nederlandse Natuurhistorische Verening (KNNV) in Wageningen. Furthermore, she was invited to give a lecture on this topic at Stichting Lingewaard Natuurlijk in Bemmel. In 2015 she started her $\mathrm{PhD}$ on congnitive consequences of natural variation in learning in parastic wasps, which has led to the development of this thesis.

In September 2019 she started working on a postdoc research project at the Netherlands Institute of ecology (NIOO-KNAW) in Wageningen. In this project she focusses on boosting the efficacy of biological control agents of citrus mealybugs through olfactory conditioning, where she continues to work with her beloved parasitoids. 



\section{Publication list}

\section{Published}

De Bruijn, J.A.C., Vet, L.E.M., Jongsma, M.A., and Smid, H.M. (2018). Automated high-throughput individual tracking system for insect behavior: Applications on memory retention in parasitic wasps. Journal of Neuroscience Methods 309, 208-217.

De Bruijn, J.A.C., Vet, L.E.M., and Smid, H.M. (2018). Costs of Persisting Unreliable Memory: Reduced Foraging Efficiency for Free-Flying Parasitic Wasps in a Wind Tunnel. Frontiers in Ecology and Evolution 6.

\section{Submitted}

De Bruijn, J.A.C., Vosteen, I., Vet, L.E.M., Smid, H.M., and de Boer, J.G. Monitoring the effects of information reliability on parasitoid foraging behaviour with a multi-camera system in the field.

De Bruijn, J.A.C., L.E.M., Smid, H.M., and de Boer, J.G. Memory extinction and spontaneous recovery shaping parasitoid foraging behaviour.

De Bruijn, J.A.C., L.E.M., Smid, H.M., and de Boer, J.G. Non-host oviposition by the parasitoid Cotesia glomerata and its effects on foraging behaviour. 


\section{PE\&RC Training and Education Statement}

With the training and education activities listed below the PhD candidate has complied with the requirements set by the C.T. de Wit Graduate School for Production Ecology and Resource Conservation (PE\&RC) which comprises of a minimum total of 32 ECTS (= 22 weeks of activities)

Review of literature (6 ECTS)

- Ecological consequences of

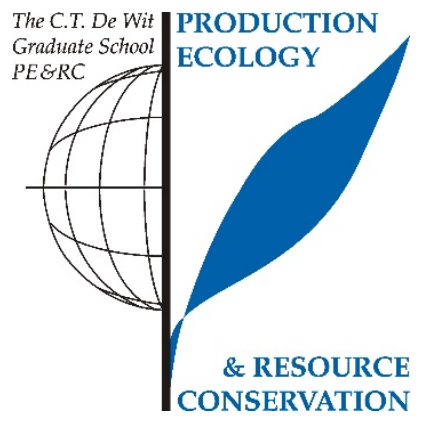

natural variation in learning rate and memory dynamics of Cotesia species

\section{Post-graduate courses (5.1 ECTS)}

- Introduction to R for statistical analysis; PE\&RC (2017)

- Multivariate analysis; PE\&RC (2017)

- $\quad$ Soil ecology; PE\&RC (2019)

- Bugs at your service; PE\&RC (2019)

Invited review of (unpublished) journal manuscript (1 ECTS)

- Scientific Reports: ecology \& behaviour (2018)

Deficiency, refresh, brush-up courses (1.5 ECTS)

- Agro-ecology in practice; De Ommuurde Tuin (2019)

Competence strengthening / skills courses (2.9 ECTS)

- Data management plan; PE\&RC (2017)

- Scientific artwork- vector graphics and images (2017)

- Scientific integrity; WGS (2019)

- Writing grant proposals; Wageningen in’to Languages (2019)

PE\&RC Annual meetings, seminars and the PE\&RC weekend (1.5 ECTS)

- PE\&RC First years weekend (2015)

- PE\&RC Last years weekend (2019)

Discussion groups / local seminars / other scientific meetings (6 ECTS)

- PhD Lunch meeting (2015-2019) 
International symposia, workshops and conferences (4.4 ECTS)

- XI European congress of entomology (2018)

- IOBC Work group meeting landscape management for functional biodiversity (2019)

Lecturing / supervision of practicals / tutorials (2.7 ECTS)

- Insect-plant interactions (2016)

- Behavioural ecology (2016-2018)

- Ecological aspects of bio-interactions (2017-2018)

\section{Supervision of MSc students}

- Sequential associative learning - extinction

- Sequential associative learning - multiple cue learning 
The research described in this thesis was financially supported by The Netherlands Organisation for Scientific Research (NWO open competition grant nr. 824.14.023 awarded to L.E.M. Vet).

Cover design and layout by the author

Printed by GVO drukkers \& vormgevers, Ede, The Netherlands 



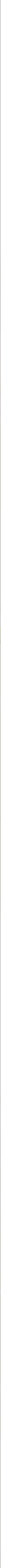




\section{Propositions}

1. Minor differences between host plant species can cause major differences in parasitoid foraging behaviour.

(this thesis)

2. Associative learning in parasitoids can lead to maladaptive foraging behaviour when learned information becomes unreliable.

(this thesis)

3. Having one child less is a highly effective way in which citizens can help mitigate climate change. (Based on: Wynes and Nicholas (2017) Environmental Research Letters 12:074024)

4. Livestock consumption is essential in a circular economy.

(Based on: Van Zanten et al. (2019) Global Food Security 21: 18-22)

5. Science grants that are eligible only for women reinforce sexism.

6. Working more than 50 hours a week hampers productivity and creativity.

Propositions belonging to the thesis entitled:

"Information reliability shaping parasitoid foraging behaviour"

Jessica A.C. de Bruijn

Wageningen, 4 February 2020 